\title{
Probabilistic interpretation for solutions of fully nonlinear stochastic PDEs
}

\author{
Anis MATOUSSI* \\ Université du Maine \\ Institut du Risque et de l'Assurance \\ Laboratoire Manceau de Mathématiques \\ e-mail: anis.matoussi@univ-lemans.fr \\ Dylan POSSAMAÏ \\ CEREMADE Université Paris-Dauphine \\ PSL Research University, CNRS \\ 75016 Paris, France \\ e-mail: possamai@ceremade.dauphine.fr \\ Wissal SABBAGH \\ Université du Maine \\ Institut du Risque et de l'Assurance \\ Laboratoire Manceau de Mathématiques \\ e-mail: wissal.sabbagh@univ-lemans.fr
}

\begin{abstract}
In this article, we propose a wellposedness theory for a class of second order backward doubly stochastic differential equation (2BDSDE). We prove existence and uniqueness of the solution under a Lipschitz type assumption on the generator, and we investigate the links between the 2BDSDEs and a class of parabolic fully nonlinear Stochastic PDEs. Precisely, we show that the Markovian solution of 2BDSDEs provide a probabilistic interpretation of the classical and stochastic viscosity solution of Fully nonlinear SPDEs.
\end{abstract}

\section{Introduction}

The starting point of this work is the following parabolic fully-nonlinear stochastic partial differential equation (SPDE for short)

$$
d u_{t}(x)+H\left(t, x, u_{t}(x), D u_{t}(, x), D^{2} u_{t}(x)\right) d t+g\left(t, x, u_{t}(x), D u_{t}(x)\right) \circ d \overleftarrow{W}_{t}=0, t \in[0, T], u_{T}=\Phi,
$$

where $g$ and $H$ are given nonlinear functions. The differential term integrated with respect to $d \overleftarrow{W}_{t}$ refers to the backward stochastic integral against a finite-dimensional Brownian motion on some probability space $\left(\Omega, \mathcal{F}, \mathbb{P},\left(W_{t}\right)_{t \geq 0}\right)$. We use the backward notation because our approach is fundamentally based on the doubly stochastic framework introduced in the seminal paper by Pardoux and Peng [52].

The class of stochastic PDEs as in (1.1) and their extensions is an important one, since it arises in a number of applications, ranging from asymptotic limits of partial differential equations (PDEs for short) with rapid (mixing) oscillations in time, phase transitions and front propagation in random media with random normal

\footnotetext{
* Research partly supported by the Chair Financial Risks of the Risk Foundation sponsored by Société Générale, the Chair Derivatives of the Future sponsored by the Fédération Bancaire Française, and the Chair Finance and Sustainable Development sponsored by EDF and Calyon
} 
velocities, filtering and stochastic control with partial observations, path-wise stochastic control theory, mathematical finance... The main difficulties with equations like (1.1) are threefold.

(i) Even in the deterministic case, there are no global smooth solutions in general.

(ii) Their fully nonlinear character seems to make them inaccessible to the classical martingale theory employed for the linear case

(iii) Even if smooth solutions were to exist, the equations cannot be described in a point-wise sense, because of the everywhere lack of differentiability of the Brownian paths.

The starting point of the theory of SPDEs was with classical solutions in a linear context, wellposedness results having been obtained notably by Pardoux [50], Dawson [20], Ichikawa [35] or Krylov and Rozovskiı̌ [38]. Extensions have been obtained later, notably by Pardoux and Peng [52] (see also Krylov and Rozovskiũ [39] or Bally and Matoussi [5]) by introducing backward doubly stochastic differential equation (BDSDE for short), which allowed them to give a nonlinear Feynman-Kac's formula for the following class of semi-linear SPDEs

$$
d u_{t}(x)+\left[\mathcal{L} u_{t}(x)+f\left(t, x, u_{t}(x), D u_{t}(x)\right)\right] d t+g\left(t, x, u_{t}(x), D u_{t}(x)\right) \circ d \overleftarrow{W}_{t}=0
$$

where $\mathcal{L}$ is a linear second order diffusion operator and $f$ is a given nonlinear function. The theory of BDSDE has then been extended in several directions, notably by Matoussi and Scheutzow [46] who considered a class of BDSDE where the nonlinear noise term is given by the more general Itô-Kunita's stochastic integral, thus allowing them to give a probabilistic interpretation of classical and Sobolev's solutions of semi-linear parabolic SPDEs driven by space-time white noise. However, the notion of viscosity solution to SPDE has remained a quite difficult and evasive subject throughout the years. Our aim in this paper, as will be detailed below, is to give a definition of a generalization of BDSDEs allowing for a probabilistic representation of solutions to fully nonlinear SPDEs, the appropriate notion of equation being that of second-order BDSDEs. Before going into details, let us review the associated literature.

Literature review for viscosity solution of SPDEs. Stochastic viscosity solutions for SPDEs were first introduced by Lions and Souganidis in their seminal papers [42, 43, 44]. They used the so-called "stochastic characteristics" to remove the stochastic integrals from the SPDEs and thus transform them into PDEs with random coefficients. A few years later, Buckdahn and Ma $[9,10]$ considered a related but different class of semi-linear SPDEs, and studied them in light of the earlier results of Lions and Souganidis, giving in addition a probabilistic interpretation of such equation via BDSDE, but only in the case where the intensity of the noise $g$ (1.2) did not depend on the gradient of the solution. They used the so-called Doss-Sussmann transformation and stochastic diffeomorphism flow technics to once more convert the semi-linear SPDEs into PDEs with random coefficients. This transformation was used again for non-standard optimal control problems in their paper [12], and also by Diehl and Friz [23] to solve semi-linear SPDEs and the associated BDSDEs driven by rough drivers. The case of fully nonlinear SPDEs was also considered by Buckdahn and Ma [11], still in the context of so-called stochastic viscosity solutions, and using a new kind of Taylor expansion for Itô-type random fields. One had then to wait for ten years to see new progresses been made. Hence, Gubinelli, Tindel and Torrecilla [31] proposed a new definition of viscosity solutions to fully nonlinear PDEs driven by a rough path via appropriate notions of test functions and rough jets. These objects were defined as a controlled processes with respect to the driving rough path, and the authors showed that their notion of solution was compatible with the seminal results of Lions and Souganidis [42,43,44] and with the recent results of Caruana, Friz and Oberhauser [15] on fully nonlinear SPDEs driven with rough drivers. Independently, a series of papers involving Buckdahn, Bulla, Ma and Zhang $[8,13,14]$ proposed yet another alternative definition, based once more on pathwise Taylor expansions 
for random fields (like in [11]), but now in the context of the functional Itô calculus of Dupire, and by identifying the solution of the SPDE to the solution of a path-dependent PDE. Finally, the recent contribution of Friz, Gassiat, Lions and Souganidis [29] extends the notion of path-wise viscosity solutions for Eikonal equations having quadratic Hamiltonians.

Literature review for 2BSDES. Motivated by numerical methods for fully nonlinear PDEs (the case when $g$ is identically null in the equation (1.1)), second order BSDEs (2BSDEs for short) were introduced by Cheridito, Soner, Touzi and Victoir in [17]. Then Soner, Touzi and Zhang [65] proposed a new formulation and obtained a complete theory of existence and uniqueness for such BSDEs. The main novelty in their approach is that they require that the solution verifies the equation $\mathbb{P}$ - a.s. for every probability measure $\mathbb{P}$ in a non-dominated class of mutually singular measures. This new point of view is inspired from the quasi-sure analysis of Denis and Martini [22] who established the connection between the so-called hedging problem in uncertain volatility models and the Black-Scholes-Barrenblatt PDE (see also Avellaneda, Levy and Paras [2] and Lyons [45]). The latter equation is fully nonlinear and has a simple piecewise linear dependance on the second order term. Intuitively speaking (we refer the reader to [65] for more details), the solution to a 2BSDE with generator $F$ and terminal condition $\xi$ can be understood as a supremum in some sense of the classical BSDEs with the same generator and terminal condition, but written under the different probability measures considered. Following this intuition, a non-decreasing process $K$ is added to the solution and it somehow pushes (in a minimal way) the solution so that it stays above the solutions of the classical BSDEs. The theory being very recent, the literature remains rather limited. However, we refer the interested reader to Possamaï [56] and Possamaï and Zhou [59] who respectively extended these wellposedness results to generators with linear and quadratic growth, as well as the recent contribution of Possamaï, Tan and Zhou [58], which lifts all the regularity assumptions assumed in the aforementioned works.

Main contributions. Our aim in this paper is to provide a complete theory of existence and uniqueness of second order BDSDEs (2BDSDEs for short) under Lipschitz-type hypotheses on the driver. In addition to the structural difficulties inherent with dealing with 2BSDEs through the quasi-sure analysis, the presence of two sources of randomness in the 2BDSDEs, which are mixed through the nonlinear coefficients of the equation, makes our study even more complex. In particular, we have to be extremely prudent when defining the probabilistic structure allowing for what is now commonly known as volatility uncertainty, in order to consider SPDEs with a nonlinearity with respect to the second-order space derivative. Note that, one of main difficulties with 2BDSDEs and BDSDEs is the extra backward integral term, which prevents us from obtaining path-wise estimates for the solutions, unlike what happens with BSDEs or 2BSDEs. This introduces additional non trivial difficulties. The same type of problems were already pointed out in [3], where the authors analyze regression schemes for approximating BDSDEs as well as their convergence, and obtain non-asymptotic error estimates, conditionally to the external noise (that is W in our context). Similarly to the classical 2BSDEs, the solution of a 2BDSDE has to be represented as a supremum of solutions to standard BDSDEs. We therefore follow the original approach of Soner, Touzi and Zhang [65] by constructing the solution path-wise, using the so-called regular conditional probability distribution. We point out that since in our context the value process is a random field depending on two source of randomness, we get a dynamic programming principle without regularity on the terminal condition and the generator following the approach of Possamaï, Tan and Zhou [58]. This is different from the classical 2BSDEs where regularity result for the value process, precisely the uniform continuity with respect to the trajectory of the fundamental noise, was crucial to prove their dynamic programming principle (Proposition 4.7 in [64]). Moreover, under regularity conditions on the coefficients, we 
show that classical solution of the fully nonlinear SPDE (1.1) can be obtained via the associated Markovian 2BDSDEs, thus extending the Feynman-Kac's formula to this context. Finally, we introduce the notion of stochastic viscosity solution for the fully non-linear SPDEs (1.1) in the case where the intensity of the noise $g$ in the SPDE (1.1) does not depend on the gradient of the solution. This restriction is due to our approach based on the Doss-Sussmann transformation to convert fully nonlinear SPDEs to fully nonlinear PDEs with random coefficients. Let us conclude by insisting on one of the main implications of our results. It is our conviction that they open a new path for possible numerical simulations of solution to fully-nonlinear SPDEs. Indeed, numerical schemes for classical BDSDEs are by now well-known (see for instance [3]), and numerical procedures for solving second-order BSDEs have also been successfully implemented in the recent years, see for instance Possamaï and Tan [57] or Ren and Tan [60]. Combining these two approaches should in principle allow to obtain efficient numerical schemes for computing solutions to 2BDSDEs, and therefore for fully non-linear SPDEs. As far as we know, there are no literature on the subject, except the cases of semilinear and quasilinear SPDEs (see [30], [32], [33], [4], [3]), and so our results could prove to be a non-negligible progress.

Structure of the paper. The paper is organized as follows. In Section 2, we recall briefly some notations, introduce the probabilistic structure on the considered product space allowing to choose the adequate set of measures, provide the precise definition of 2BDSDEs and show how they are connected to classical BDSDEs. Then, the aim of Section 3 is to prove the uniqueness of solution for 2BDSDEs, as a direct consequence of a representation theorem, which intuitively originates from the stochastic control interpretation of our problem. The proof of this representation is based on Lemma 3.1. In this section, we prove also a priori estimates for 2BDSDEs. Section 4 is devoted to the existence result for solution of 2BDSDEs by a path-wise construction on the shifted Wiener space. Since in our context, the value process is a random field depending on two source of randomness, we prove its regularity result in Lemma 4.2. Once again, we cannot obtain the same regularity in the context of doubly stochastic 2BSDEs because we cannot have path-wise estimates for their solutions. In Section 5, we specialize our discussion to the Markovian context, and we give in Theorem 5.1 the FeynmanKac's formula for classical solution of such SPDEs. Then, we introduce the notion of stochastic viscosity solution and give in Theorem 5.3 the probabilistic representation for such solution, which is in our knowledge the first result of the kind for such class of fully nonlinear SPDEs. Finally, the Appendix collects several technical results needed for the existence of the solution of the 2BDDSEs and SPDEs.

Notations: For any $n \in \mathbb{N} \backslash\{0\}$, we will denote by $x \cdot y$ the usual inner product of two elements $(x, y)$ of $\mathbb{R}^{n}$, and by $\|\cdot\|$ the associated Euclidean norm when $n \geq 2$ and by $|\cdot|$ when $n=1$. Furthermore, for any $n \times n$ matrix with real entries $M, M^{\top}$ will denote its usual transpose. We abuse notations and also denote by $\|\cdot\|$ a norm on the space of square matrices with real entries. Moreover, $\mathbb{S}_{n}^{>0}$ will denote the space of all $n \times n$ positive definite matrices with real entries. For any topological space $E, \mathcal{B}(E)$ will denote the associated Borel $\sigma$-field.

\section{Preliminaries and assumptions}

Let us fix a positive real number $T>0$, which will be our finite time horizon, as well as some integer $d \geq 1$. We shall work on the product space $\Omega:=\Omega^{B} \times \Omega^{W}$ where

- $\Omega^{B}$ is the canonical space of continuous functions on $[0, T]$ vanishing at 0 , equipped with the uniform norm $\|\cdot\|_{\infty}$. $B$ will be the canonical process on $\Omega^{B}$, and $\mathbb{P}_{B}^{0}$ the Wiener measure on $\left(\Omega^{B}, \mathcal{F}^{B}\right)$, where $\mathcal{F}^{B}$ is the Borel $\sigma$-algebra. Generically, we will denote by $\omega^{B}$ an element of $\Omega^{B}$. 
- $\left(\Omega^{W}, \mathcal{F}^{W}, \mathbb{P}_{W}^{0}\right)$ is a copy of $\left(\Omega^{B}, \mathcal{F}^{B}\right)$ whose canonical process is denoted by $W$, and whose Wiener measure is denoted bu $\mathbb{P}_{0}^{W}$. Generically, we will denote by $\omega^{W}$ an element of $\Omega^{W}$, and the notation $\omega$ will be solely reserved for elements $\omega:=\left(\omega^{B}, \omega^{W}\right)$ of $\Omega$.

We equip the product space $\Omega$ with the product $\sigma$-algebra $\mathcal{F}=\mathcal{F}^{B} \otimes \mathcal{F}^{W}$, and define $\mathbb{P}_{0}:=\mathbb{P}_{B}^{0} \otimes \mathbb{P}_{W}^{0}$. Let then $\mathbb{F}^{o, W}:=\left\{\mathcal{F}_{t, T}^{o, W}\right\}_{t \geq 0}$ and $\mathbb{F}^{W}:=\left\{\mathcal{F}_{t, T}^{W}\right\}_{t \geq 0}$ be respectively the natural and the augmented (under $\mathbb{P}_{W}^{0}$ ) retrograde filtration generated by $W$, defined by

$$
\mathcal{F}_{s, t}^{o, W}:=\sigma\left\{W_{r}-W_{s}, s \leq r \leq t\right\}, \mathcal{F}_{s, t}^{W}:=\sigma\left\{W_{r}-W_{s}, s \leq r \leq t\right\} \vee \mathcal{N}^{\mathbb{P}_{W}^{0}}\left(\mathcal{F}^{W}\right),
$$

where

$$
\mathcal{N}^{\mathbb{P}_{W}^{0}}\left(\mathcal{F}^{W}\right):=\left\{A \in \Omega^{W}, \exists \tilde{A} \in \mathcal{F}^{W}, A \subset \tilde{A} \text { and } \mathbb{P}_{W}^{0}(\tilde{A})=0\right\} .
$$

We also denote for simplicity $\mathcal{F}_{T}^{W}:=\mathcal{F}_{0, T}^{W}$. Similarly, we let $\mathbb{F}^{B}:=\left\{\mathcal{F}_{t}^{B}\right\}_{t \geq 0}$ be the forward (raw) filtration generated by $B$, that is $\mathcal{F}_{t}^{B}:=\sigma\left\{B_{r}, 0 \leq r \leq t\right\}$ (we remind the reader that it is a classical result that in this case $\left.\mathcal{F}^{B}=\mathcal{F}_{T}^{B}\right)$. We also consider its right limit $\mathbb{F}_{+}^{B}:=\left\{\mathcal{F}_{t^{+}}^{B}\right\}_{t \geq 0}$. Finally, for each $t \in[0, T]$, we define

$$
\mathcal{F}_{t}:=\mathcal{F}_{t}^{B} \vee \mathcal{F}_{t, T}^{W} \text { and } \quad \mathcal{G}_{t}:=\mathcal{F}_{t}^{B} \vee \mathcal{F}_{T}^{W}
$$

The collection $\mathbb{F}=\left(\mathcal{F}_{t}\right)_{0 \leq t \leq T}$ is neither increasing nor decreasing and therefore does not constitute a filtration. However, $\mathbb{G}=\left(\mathcal{G}_{t}\right)_{0 \leq t \leq T}$ is a filtration.

For technical reasons related to the main result of Nutz [47], we will work under the set theoretic model of ZFC (Zermelo-Fraenkel plus the axiom of choice) as well as any additional axiom ensuring the existence of medial limits in the sense of Mokobodzki (see [28], statement 22O(1) page 55 for models ensuring this).

\subsection{A special family of measures on $(\Omega, \mathcal{F})$}

In order to be able to consider SPDEs with a nonlinearity with respect to the second-order space derivative, we will need to consider a probabilistic structure allowing for what is now commonly known as volatility uncertainty. This basically means that we will allow the probability measure we consider on $\left(\Omega^{B}, \mathcal{F}^{B}\right)$ to change. Such an approach has been initiated with the name of quasi-sure stochastic analysis by Denis and Martini [22] and has since proved very successful (see among others $[64,65,48]$ ). Following this approach, we say that a probability measure $\mathbb{P}_{B}$ on $\left(\Omega^{B}, \mathcal{F}^{B}\right)$ is a local martingale measure if the canonical process $B$ is a local martingale under $\mathbb{P}_{B}$. We emphasize that by using integration by parts as well as the path-wise stochastic integration of Bichteler (see Theorem 7.14 in [7] or the more recent article of Karandikar [36]), we can give a path-wise definition of the quadratic variation $\langle B\rangle_{t}$ and its density with respect to the Lebesgue measure $\widehat{a}_{t}$, by

$$
\widehat{a}_{t}:=\varlimsup_{\epsilon \downarrow 0} \frac{1}{\epsilon}\left(\langle B\rangle_{t}-\langle B\rangle_{t-\epsilon}\right) .
$$

where the $\varlimsup$ lim has to be understood in a component-wise sense.

For practical purposes, we will restrict our attention to the set $\overline{\mathcal{P}}_{S}$ consisting of all probability measures

$$
\mathbb{P}(A):=\int_{\Omega^{W}} \int_{\Omega^{B}} \mathbf{1}_{\left(\omega^{B}, \omega^{W}\right) \in A} d \mathbb{P}^{\alpha, \omega^{W}}\left(\omega^{B}\right) d \mathbb{P}_{W}^{0}\left(\omega^{W}\right), A \in \mathcal{F},
$$

such that for any $\omega^{W} \in \Omega^{W}$

$$
\mathbb{P}^{\alpha, \omega^{W}}:=\mathbb{P}_{B}^{0} \circ\left(X^{\alpha, \omega^{W}}\right)^{-1}, \text { where } X_{t}^{\alpha, \omega^{W}}:=\int_{0}^{t} \alpha_{s}^{1 / 2}\left(\cdot, \omega^{W}\right) d B_{s}, t \in[0, T], \mathbb{P}_{B}^{0}-\text { a.s. },
$$


for some $\mathbb{F}$-adapted process $\alpha$ taking values in $\mathbb{S}_{d}^{>0}$ and satisfying for any $\omega^{W} \in \Omega^{W}$,

$$
\int_{0}^{T}\left\|\alpha_{t}\left(\cdot, \omega^{W}\right)\right\| d t<\infty, \mathbb{P}_{B}^{0}-a . s .
$$

We emphasize here that by the classical results of Stricker and Yor [66] on stochastic integration with a parameter, that we can always assume without loss of generality that the map

$$
\left(t, \omega^{B}, \omega^{W}\right) \longmapsto\left(\int_{0}^{t} \alpha_{s}\left(\cdot, \omega^{W}\right)^{1 / 2} d B_{s}\right)\left(\omega^{B}\right),
$$

is $\mathcal{B}([0, t]) \otimes \mathcal{F}_{t}$-measurable. This implies in particular that the family $\left(\mathbb{P}^{\alpha, \omega^{W}}, \omega^{W} \in \Omega^{W}\right)$ is a stochastic kernel (see for instance Definition 7.12 in [6]). The set $\overline{\mathcal{P}}_{S}$ has several nice properties, which can be deduced from similar results in [63].

Lemma 2.1. Every $\mathbb{P} \in \overline{\mathcal{P}}_{S}$ satisfies the martingale representation property, in the sense that for any square integrable $(\mathbb{P}, \mathbb{G})$-martingale $M$, there exists a unique $\mathbb{G}$-predictable process $Z$ such that

$$
M_{t}=M_{0}+\int_{0}^{t} Z_{s} \cdot d B_{s}, \mathbb{P}-a . s .
$$

Moreover, they satisfy the Blumenthal $0-1$ law, and in particular, any $\mathcal{G}_{0^{+}}$or $\mathcal{F}_{0^{+}}-$measurable random variable is deterministic with respect to $\Omega^{B}$, that is its randomness only comes from $\Omega^{W}$.

Proof. Let $M$ be a square integrable $(\mathbb{P}, \mathbb{G})$-martingale. We have

$$
M_{s}\left(\omega^{B}, \omega^{W}\right)=\mathbb{E}^{\mathbb{P}}\left[M_{t} \mid \mathcal{G}_{s}\right]\left(\omega^{B}, \omega^{W}\right), 0 \leq s \leq t \leq T, \mathbb{P}-\text { a.e. }\left(\omega^{B}, \omega^{W}\right) \in \Omega .
$$

However, using again the result of Stricker and Yor [66], this can be rewritten, for $\mathbb{P}_{0}^{W}-$ a.e. $\omega^{W} \in \Omega^{W}$, as

$$
M_{s}\left(\cdot, \omega^{W}\right)=\mathbb{E}^{\mathbb{P}^{\alpha, \omega} W}\left[M\left(\cdot, \omega^{W}\right) \mid \mathcal{F}_{t}^{B}\right](\cdot), \mathbb{P}^{\alpha, \omega^{W}}-\text { a.s. },
$$

which therefore implies that for $\mathbb{P}_{0}^{W}$ - a.e. $\omega^{W} \in \Omega^{W}, M\left(\cdot, \omega^{W}\right)$ is a $\left(\mathbb{P}^{\alpha, \omega^{W}}, \mathbb{F}^{B}\right)$-martingale, to which we can then apply the result of [63] to obtain the required martingale representation.

The exact same reasoning gives us the second desired result, using again the fact that by [63], the probability measures $\mathbb{P}^{\alpha, \omega^{W}}$ satisfy the Blumenthal $0-1$ law.

Remark 2.1. We recall from [64] that for a fixed $\mathbb{P} \in \overline{\mathcal{P}}_{S}$, we have from the Blumenthal zero-one law that $\mathbb{E}^{\mathbb{P}}\left[\xi \mid \mathcal{G}_{t}\right]=\mathbb{E}^{\mathbb{P}}\left[\xi \mid \mathcal{G}_{t^{+}}\right], \mathbb{P}$ - a.s. for any $t \in[0, T]$ and $\mathbb{P}$-integrable $\xi$. In particular, this implies immediately that any $\mathcal{G}_{t^{+}}$-measurable random variable has a $\mathcal{G}_{t}$-measurable $\mathbb{P}$-modification. Furthermore, if $\mathbb{F}^{W, o}$ denotes the raw backward filtration of the Brownian motion $W$, then any $\mathcal{G}_{t^{+}}-$measurable random variable also admits a $\mathbb{F}_{t}^{B} \vee \mathcal{F}_{T}^{W, o}$-measurable $\mathbb{P}$-modification. We will often implicitly work with such modifications (which of course depend on the considered measure $\mathbb{P})$.

We finish this section with the following definition.

Definition 2.1. For any subset $\mathcal{Q}$ of $\overline{\mathcal{P}}_{S}$, we say that a property holds $\mathcal{Q}$-quasi-surely $(\mathcal{Q}-$ q.s. for short) if it holds $\mathbb{P}-$ a.s. for all $\mathbb{P} \in \mathcal{Q}$. 


\subsection{The non-linearity}

To introduce the non-linearity in the SPDEs we consider, we have to take a small detour, and start by introducing a map $H_{t}(w, y, z, \gamma):[0, T] \times \Omega \times \mathbb{R} \times \mathbb{R}^{d} \times D_{H} \longrightarrow \mathbb{R}$, where $D_{H} \subset \mathbb{R}^{d \times d}$ is a given subset containing 0 . As is usual in any stochastic control problem, the following Fenchel conjugate of $H$ with respect to $\gamma$ will play an important role

$$
F_{t}(w, y, z, a):=\sup _{\gamma \in D_{H}}\left\{\frac{1}{2} \operatorname{Tr}(a \gamma)-H_{t}(w, y, z, \gamma)\right\} \text { for } a \in \mathbb{S}_{d}^{>0} .
$$

For ease of notations, we also define

$$
\widehat{F}_{t}(y, z):=F_{t}\left(y, z, \widehat{a}_{t}\right) \text { and } \widehat{F}_{t}^{0}:=\widehat{F}_{t}(0,0)
$$

where as usual we abuse notations and suppress the dependence on $\omega \in \Omega$ when it is not important (which will not be the case in all the article). Since $F$ may not be always finite, we denote by $D_{F_{t}(y, z)}:=\left\{a, F_{t}(w, y, z, a)<+\infty\right\}$ the domain of $F$ in $a$ for a fixed $(t, w, y, z)$.

We will also consider a function $g_{t}(\omega, y, z):[0, T] \times \Omega \times \mathbb{R} \times \mathbb{R}^{d} \longrightarrow \mathbb{R}^{d}$ and denote $g_{t}^{0}:=g_{t}(0,0)$, with the same convention as above on the $\omega$-dependence. The maps $\widehat{F}$ and $g$ are intended to play the role of the generators of the doubly stochastic BSDEs we will consider later on. Since the theory of BDSDEs is an $L^{2}$-type theory, we need to restrict again the class $\overline{\mathcal{P}}_{S}$ to account for these integrability issues.

Definition 2.2. $\mathcal{P}$ is the collection of all $\mathbb{P} \in \overline{\mathcal{P}}_{S}$ such that

$$
\begin{gathered}
\underline{a}_{\mathbb{P}} \leq \widehat{a} \leq \bar{a}_{\mathbb{P}}, d t \times d \mathbb{P}-\text { a.e. for some } \underline{a}_{\mathbb{P}}, \bar{a}_{\mathbb{P}} \in \mathbb{S}_{d}^{>0}, \\
\mathbb{E}^{\mathbb{P}}\left[\left(\int_{0}^{T}\left|\widehat{F}_{t}^{0}\right|^{2} d t\right)\right]<+\infty, \mathbb{E}^{\mathbb{P}}\left[\left(\int_{0}^{T}\left\|g_{t}^{0}\right\|^{2} d t\right)\right]<+\infty .
\end{gathered}
$$

The first condition in Definition 2.2 ensures that the process $B$ is actually a square-integrable martingale under any of the measures in $\mathcal{P}$, and not only a local-martingale, while the second condition is here to ensure wellposedness of the BDSDEs which will be defined below. Of course, these integrability assumptions will not be enough and need to be complemented with further assumptions on the functions $F$ and $g$ that we now list

Assumption 2.1. (i) $\mathcal{P}$ is not empty, and the domain $D_{F_{t}(y, z)}=: D_{F_{t}}$ is actually independent of $(\omega, y, z)$.

(ii) For fixed $(t, y, z, a) \in[0, T] \times \mathbb{R} \times \mathbb{R}^{d} \times D_{F_{t}}, F_{t}(\cdot, y, z, a)$ is $\mathcal{F}_{t}$-measurable, and $g$ is $\mathcal{F}_{t}$-measu-rable as well.

(iii) There is $C>0$ and $0 \leq \alpha<1$ s.t. $\forall\left(y, y^{\prime}, z, z^{\prime}, t, a, \omega\right) \in \mathbb{R} \times \mathbb{R} \times \mathbb{R}^{d} \times \mathbb{R}^{d} \times[0, T] \times D_{F_{t}} \times \Omega$,

$$
\begin{gathered}
\left|F_{t}(\omega, y, z, a)-F_{t}\left(\omega, y^{\prime}, z^{\prime}, a\right)\right| \leq C\left(\left|y-y^{\prime}\right|+\left\|a^{1 / 2}\left(z-z^{\prime}\right)\right\|\right), \\
\left\|g_{t}(\omega, y, z)-g_{t}\left(\omega, y^{\prime}, z^{\prime}\right)\right\|^{2} \leq C\left|y-y^{\prime}\right|^{2}+\alpha\left\|z-z^{\prime}\right\|^{2}
\end{gathered}
$$

(iv) There exists a constant $\lambda \in[0,1[$ such that

$$
(1-\lambda) \widehat{a}_{t} \geq \alpha I_{d}, d t \times \mathcal{P}-q . e .
$$

(v) $F$ and $g$ are uniformly continuous in $\omega$ for the $\|\cdot\|_{\infty}$ norm on $\Omega$. 
Remark 2.2. The assumptions (i) and (ii) are classic in the second order framework, see [65]. The Lipschitz assumption (iii) is standard in the BSDE theory since the paper [51]. The contraction condition satisfied by $g$ with respect to the variable $z$ (i.e $0 \leq \alpha<1$ ) and assumption (iv) are necessary for the wellposedness of our second order BDSDEs. These type of conditions are well known (see e.g. [50]) for the semilinear stochastic PDE (1.2) to be a well-posed stochastic parabolic equation. The last hypothesis $(v)$ is proper to the second order framework, it is linked to our intensive use of regular conditional probability distributions (r.c.p.d.) in our existence proof, and to the fact that we construct our solutions pathwise, thus avoiding complex issues related to negligible sets.

\subsection{Important spaces and norms}

For the formulation of the second order BDSDEs, we will use the same spaces and norms (albeit with some modifications, in particular concerning the measurability assumptions) as the one introduced for second order BSDEs in [65].

- For $p \geq 1, L^{p}$ denotes the space of all $\mathcal{F}_{T}$-measurable scalar r.v. $\xi$ with

$$
\|\xi\|_{L^{p}}^{p}:=\sup _{\mathbb{P} \in \mathcal{P}} \mathbb{E}^{\mathbb{P}}\left[|\xi|^{p}\right]<+\infty .
$$

- $\mathbb{H}^{p}$ denotes the space of all $\mathbb{R}^{d}$-valued processes $Z$ which are $\mathbb{G}$-predictable and s.t. $Z_{t}$ is $\underset{\mathbb{P} \in \mathcal{P}}{\bigcap} \overline{\mathcal{F}}_{t}^{\mathbb{P}}-$ measurable for a.e. $t \in[0, T]$, with

$$
\|Z\|_{\mathbb{H}^{p}}^{p}:=\sup _{\mathbb{P} \in \mathcal{P}} \mathbb{E}^{\mathbb{P}}\left[\left(\int_{0}^{T}\left\|\widehat{a}_{t}^{1 / 2} Z_{t}\right\|^{2} d t\right)^{\frac{p}{2}}\right]<+\infty .
$$

- $\mathbb{D}^{p}$ denotes the space of $\mathbb{R}$-valued processes $Y$, which are $\mathbb{G}$-progressively measurable, s.t. $Y_{t}$ is $\mathcal{F}_{t^{+}}^{B} \vee \mathcal{F}_{t, T}^{W}-$ measurable for every $t \in[0, T]$, with

$$
\mathcal{P} \text { - q.s. càdlàg paths, and }\|Y\|_{\mathbb{D}^{p}}^{p}:=\sup _{\mathbb{P} \in \mathcal{P}} \mathbb{E}^{\mathbb{P}}\left[\sup _{0 \leq t \leq T}\left|Y_{t}\right|^{p}\right]<+\infty .
$$

- $\mathbb{I}^{p}$ denotes the space of all $\mathbb{R}$-valued and $\mathbb{G}$-progressively measurable processes $K$ null at 0 , s.t. $K_{t}$ is $\bigcap_{\mathbb{P} \in \mathcal{P}} \overline{\mathcal{F}}_{t}^{\mathbb{P}}$-measurable for every $t \in[0, T]$, with

$$
\mathcal{P} \text { - q.s. càdlàg and nondecreasing paths, and }\|K\|_{\mathbb{I}^{p}}^{p}:=\sup _{\mathbb{P} \in \mathcal{P}} \mathbb{E}^{\mathbb{P}}\left[K_{T}^{p}\right]<+\infty \text {. }
$$

- For each $\xi \in L^{1}, \mathbb{P} \in \mathcal{P}$ and $t \in[0, T]$, we denote by $\mathbb{E}_{t}^{\mathcal{P}, \mathbb{P}}[\xi]:=\underset{\mathbb{P}^{\prime} \in \mathcal{P}\left(t^{+}, \mathbb{P}\right)}{\operatorname{essup}} \mathbb{E}_{t}^{\mathbb{P}^{\prime}}[\xi]$, with

$$
\mathcal{P}\left(t^{+}, \mathbb{P}\right):=\left\{\mathbb{P}^{\prime} \in \mathcal{P}, \mathbb{P}^{\prime}=\mathbb{P} \text { on } \mathcal{G}_{t^{+}}\right\},
$$

where $\mathbb{E}_{t}^{\mathbb{P}}[\xi]:=\mathbb{E}^{\mathbb{P}}\left[\xi \mid \mathcal{G}_{t}\right]=\mathbb{E}^{\mathbb{P}}\left[\xi \mid \mathcal{F}_{t}^{B} \vee \mathcal{F}_{T}^{W}\right], \mathbb{P}-$ a.s. Then we define for each $p \geq 2$,

$$
\mathbb{L}^{p}:=\left\{\xi \in L^{p}:\|\xi\|_{\mathbb{L}^{p}}<+\infty\right\},
$$

where

$$
\|\xi\|_{\mathbb{L}^{p}}^{p}:=\sup _{\mathbb{P} \in \mathcal{P}} \mathbb{E}^{\mathbb{P}}\left[\operatorname{ess~sup}_{0 \leq t \leq T}^{\mathbb{P}}\left(\mathbb{E}_{t}^{\mathcal{P}, \mathbb{P}}\left[|\xi|^{2}\right]\right)^{\frac{p}{2}}\right] .
$$

Finally, we denote by $\mathrm{UC}_{b}(\Omega)$ the collection of all bounded and uniformly continuous maps $\xi: \Omega \longrightarrow \mathbb{R}$ for the $\|\cdot\|_{\infty}-$ norm, and we let $\mathcal{L}^{p}$ be the closure of $\mathrm{UC}_{b}(\Omega)$ under the norm $\|\cdot\|_{\mathbb{L}^{p}}$, for every $p \geq 2$. 


\subsection{Definition of the 2BDSDE and connection with standard BDSDEs}

We shall consider the following second order backward doubly stochastic differential equation (2BDSDE for short)

$$
Y_{t}=\xi+\int_{t}^{T} \widehat{F}_{s}\left(Y_{s}, Z_{s}\right) d s+\int_{t}^{T} g_{s}\left(Y_{s}, Z_{s}\right) \cdot d \overleftarrow{W}_{s}-\int_{t}^{T} Z_{s} \cdot d B_{s}+K_{T}-K_{t}
$$

We note that the integral with respect to $W$ is a "backward Itô integral" (see [40], pages 111-112) and the integral with respect to $B$ is a standard forward Itô integral. For any $\mathbb{P} \in \mathcal{P}, \mathbb{G}$-stopping time $\tau$, and $\mathcal{G}_{\tau}$-measurable random variable $\xi \in \mathbb{L}^{2}(\mathbb{P})$, let $\left(y^{\mathbb{P}}, z^{\mathbb{P}}\right):=\left(y^{\mathbb{P}}(\tau, \xi), z^{\mathbb{P}}(\tau, \xi)\right)$ denote the unique solution to the following BDSDE

$$
y_{t}^{\mathbb{P}}=\xi+\int_{t}^{\tau} \widehat{F}_{s}\left(y_{s}^{\mathbb{P}}, z_{s}^{\mathbb{P}}\right) d s+\int_{t}^{\tau} g_{s}\left(y_{s}^{\mathbb{P}}, z_{s}^{\mathbb{P}}\right) \cdot d \overleftarrow{W}_{s}-\int_{t}^{\tau} z_{s}^{\mathbb{P}} \cdot d B_{s}, 0 \leq t \leq T, \mathbb{P}-\text { a.s } .
$$

Let us point out immediately that wellposedness of a solution is not an immediate consequence of the classical result of Pardoux and Peng [52]. Indeed, in our setting the two martingales $W$ and $B$ are not independent. In our case, the only argument in [52] which does not go through mutatis mutandis is the one at the end of their proof of their Proposition 1.2 which proves that the solution $\left(y_{t}^{\mathbb{P}}, z_{t}^{\mathbb{P}}\right)$ is actually $\mathcal{F}_{t^{+}}^{B} \vee \mathcal{F}_{t, T}^{W}$-measurable. However, by Lemma 4.1 and Step 1 of its proof, in particular (A.4), the required measurability becomes clear.

We can now give the definition of a solution to a 2BDSDE.

Definition 2.3. For $\xi \in \mathbb{L}^{2},(Y, Z, K) \in \mathbb{D}^{2} \times \mathbb{H}^{2} \times \mathbb{I}^{2}$ is a solution to the 2BDSDE (2.1) if (2.1) is satisfied $\mathcal{P}-$ q.s., and if the process $K$ satisfies the following minimality condition

$$
K_{t}=\underset{\mathbb{P}^{\prime} \in \mathcal{P}\left(t^{+}, \mathbb{P}\right)}{\operatorname{essinf}} \mathbb{E}_{t}^{\mathbb{P}^{\prime}}\left[K_{T}\right], \mathbb{P}-\text { a.s. for all } \mathbb{P} \in \mathcal{P}, t \in[0, T] .
$$

Remark 2.3. We emphasize that we should normally make the dependence of $K$ in the measure $\mathbb{P}$ explicit, since the two stochastic integrals on the right-hand side of (2.1) are, a priori, only defined $\mathbb{P}-$ a.s. However, we are in a context where we can use the main aggregation result of [47] to always define an universal version of these integrals.

Before closing this subsection, we highlight the fact that Definition 2.3 contains the classical theory of BDSDEs. Indeed, let $f$ be the following linear function of $\gamma$

$$
f_{t}(y, z, \gamma)=\frac{1}{2} I_{d}: \gamma-\hat{f}_{t}(y, z)
$$

where $I_{d}$ is the identity matrix in $\mathbb{R}^{d}$. Then, we verify immediately that $D_{F_{t}(w)}=\left\{I_{d}\right\}, \widehat{F}_{t}(y, z)=\hat{f}_{t}(y, z)$ and $\mathcal{P}=\left\{\mathbb{P}_{0}\right\}$. In this case, the minimum condition (2.3) implies

$$
0=K_{0}=\mathbb{E}^{\mathbb{P}_{0}}\left[K_{T}\right] \text { and thus } K=0, \mathbb{P}_{0}-\text { a.s. },
$$

since $K$ is nondecreasing. Hence, the 2BDSDE (2.1) is equivalent to the following BDSDE:

$$
Y_{t}=\xi+\int_{t}^{T} \hat{f}_{s}\left(Y_{s}, Z_{s}\right) d s+\int_{t}^{T} g_{s}\left(Y_{s}, Z_{s}\right) \cdot d \overleftarrow{W}_{s}-\int_{t}^{T} Z_{s} \cdot d B_{s}, 0 \leq t \leq T, \mathbb{P}_{0}-\text { a.s. }
$$

In addition to Assumption 2.1, we will need to assume the following stronger integrability conditions

Assumption 2.2. The processes $\widehat{F}^{0}$ and $g^{0}$ satisfy the following integrability conditions for some $\varepsilon>0$

$$
\phi^{2, \varepsilon}:=\sup _{\mathbb{P} \in \mathcal{P}} \mathbb{E}^{\mathbb{P}}\left[\int_{0}^{T}\left|\widehat{F}_{s}^{0}\right|^{2+\varepsilon} d s\right]<+\infty, \psi^{2, \varepsilon}:=\sup _{\mathbb{P} \in \mathcal{P}} \mathbb{E}^{\mathbb{P}}\left[\int_{0}^{T}\left\|g_{s}^{0}\right\|^{2+\varepsilon} d s\right]<+\infty .
$$


Finally, we will see later on in our proof of a priori estimates for the solution of the 2BDSDE (2.1), that we actually need to have $L^{2+\varepsilon}$-type estimates for the solutions $\left(y^{\mathbb{P}}, z^{\mathbb{P}}\right)$ of the corresponding BDSDEs. For this reason, we need to also consider the following, which already appeared as Assumption (H.2) in [52]

Assumption 2.3. There exist $c>0$ and $0 \leq \beta<1$ such that for all $(t, y, z) \in[0, T] \times \mathbb{R} \times \mathbb{R}^{d}$

$$
\left(g_{t} g_{t}^{\top}\right)(y, z) \leq c\left(1+y^{2}\right) I_{d}+\beta z z^{\top} .
$$

\section{Uniqueness of the solution and estimates}

\subsection{Representation and uniqueness of the solution}

The aim of this section is to prove the uniqueness of solution for 2BDSDEs (2.1), as a direct consequence of a representation theorem, which intuitively originates from the stochastic control interpretation of our problem. As it will become more and more apparent, one of main difficulties with 2BDSDEs and BDSDEs is the extra backward integral term, which prevents us from obtaining pathwise estimates for the solutions, unlike what happens with BSDEs or 2BSDEs. This introduces additional non trivial difficulties. The same type of problems were already pointed out in [3], where the authors analyze regression schemes for approximating BDSDEs as well as their convergence, and obtain non-asymptotic error estimates, conditionally to the external noise (that is $W$ in our context). We start with a revisit of the minimality condition (2.3).

Lemma 3.1. The minimum condition (2.3) implies that

$$
\inf _{\mathbb{P}^{\prime} \in \mathcal{P}\left(t^{+}, \mathbb{P}\right)} \mathbb{E}^{\mathbb{P}^{\prime}}\left[K_{T}-K_{t}\right]=0
$$

Proof. Indeed, fix some $\mathbb{P} \in \mathcal{P}$ and some $\mathbb{P}^{\prime} \in \mathcal{P}\left(t^{+}, \mathbb{P}\right)$. Taking expectation under $\mathbb{P}$ in (2.3), we obtain readily

$$
\mathbb{E}^{\mathbb{P}}\left[\underset{\mathbb{P}^{\prime} \in \mathcal{P}\left(t^{+}, \mathbb{P}\right)}{\operatorname{essinf}} \mathbb{E}_{t}^{\mathbb{P}} \mathbb{P}^{\mathbb{P}^{\prime}}\left[K_{T}-K_{t}\right]\right]=0
$$

Then, we know that the family $\mathcal{P}\left(t^{+}, \mathbb{P}\right)$ is upward directed (it is indeed clear from the result of [64]). Therefore, by classical results, there is a sequence $\left(\mathbb{P}^{n}\right)_{n \geq 0} \subset \mathcal{P}\left(t^{+}, \mathbb{P}\right)$ such that

$$
\underset{\mathbb{P}^{\prime} \in \mathcal{P}\left(t^{+}, \mathbb{P}\right)}{\operatorname{essinf}} \mathbb{E}_{t}^{\mathbb{P}^{\prime}}\left[K_{T}-K_{t}\right]=\lim _{n \rightarrow+\infty} \downarrow \mathbb{E}_{t}^{\mathbb{P}^{n}}\left[K_{T}-K_{t}\right] .
$$

Using this in (3.1) and then the monotone convergence theorem under the fixed measure $\mathbb{P}$, we obtain

$$
\begin{aligned}
0=\mathbb{E}^{\mathbb{P}}\left[\operatorname{essinf}_{\mathbb{P}^{\prime} \in \mathcal{P}\left(t^{+}, \mathbb{P}\right)}^{\mathbb{P}} \mathbb{E}_{t}^{\mathbb{P}^{\prime}}\left[K_{T}-K_{t}\right]\right]=\mathbb{E}^{\mathbb{P}}\left[\lim _{n \rightarrow+\infty} \downarrow \mathbb{E}_{t}^{\mathbb{P}^{n}}\left[K_{T}-K_{t}\right]\right] & =\lim _{n \rightarrow+\infty} \downarrow \mathbb{E}^{\mathbb{P}}\left[\mathbb{E}_{t}^{\mathbb{P}^{n}}\left[K_{T}-K_{t}\right]\right] \\
& =\lim _{n \rightarrow+\infty} \downarrow \mathbb{E}^{\mathbb{P}^{n}}\left[\mathbb{E}_{t}^{\mathbb{P}^{n}}\left[K_{T}-K_{t}\right]\right] \\
& =\lim _{n \rightarrow+\infty} \downarrow \mathbb{E}^{\mathbb{P}^{n}}\left[K_{T}-K_{t}\right] \\
& \geq \inf _{\mathbb{P}^{\prime} \in \mathcal{P}\left(t^{+}, \mathbb{P}\right)} \mathbb{E}^{\mathbb{P}^{\prime}}\left[K_{T}-K_{t}\right] .
\end{aligned}
$$

Since $K$ is a non-decreasing process, the result follows.

We can now show as in Theorem 4.4 of [64] that the solution to the 2BDSDE (2.1) can be represented as a supremum of solutions to the BDSDEs (2.2). 
Theorem 3.1. Let Assumptions 2.1 and 2.2 hold. Assume $\xi \in \mathbb{L}^{2}$ and that $(Y, Z, K)$ is a solution to 2BDSDE (2.1). Then, for any $\mathbb{P} \in \mathcal{P}$ and $0 \leq t_{1}<t_{2} \leq T$,

$$
Y_{t_{1}}=\underset{\mathbb{P}^{\prime} \in \mathcal{P}\left(t_{1}^{+}, \mathbb{P}\right)}{\operatorname{ess} \sup ^{\mathbb{P}}} y_{t_{1}}^{\mathbb{P}^{\prime}}\left(t_{2}, Y_{t_{2}}\right), \mathbb{P}-\text { a.s. }
$$

Proof. We follow the (by now) classical approach for this problem, first used in [65], and proceed in 2 steps.

(i) Fix $0 \leq t_{1}<t_{2} \leq T$, and $\mathbb{P} \in \mathcal{P}$. For any $\mathbb{P}^{\prime} \in \mathcal{P}\left(t_{1}^{+}, \mathbb{P}\right)$, note that from $(2.1)$, we have, $\mathbb{P}^{\prime}-$ a.s., for any $t_{1} \leq t \leq t_{2}$

$$
Y_{t}=Y_{t_{2}}+\int_{t}^{t_{2}} \widehat{F}_{s}\left(Y_{s}, Z_{s}\right) d s+\int_{t}^{t_{2}} g_{s}\left(Y_{s}, Z_{s}\right) \cdot d \overleftarrow{W}_{s}-\int_{t}^{t_{2}} Z_{s} \cdot d B_{s}+K_{t_{2}}-K_{t}
$$

and that $K$ is nondecreasing, $\mathbb{P}^{\prime}-$ a.s. Applying the comparison principle for BDSDE (see [61]) under $\mathbb{P}$, we have $Y_{t_{1}} \geq y_{t_{1}}^{\mathbb{P}^{\prime}}\left(t_{2}, Y_{t_{2}}\right), \mathbb{P}^{\prime}-$ a.s. Since $\mathbb{P}^{\prime}=\mathbb{P}$ on $\mathcal{G}_{t_{1}}^{+}$, we get $Y_{t_{1}} \geq y_{t_{1}}^{\mathbb{P}^{\prime}}\left(t_{2}, Y_{t_{2}}\right), \mathbb{P}-$ a.s. and thus

$$
Y_{t_{1}} \geq \underset{\mathbb{P}^{\prime} \in \mathcal{P}\left(t_{1}^{+}, \mathbb{P}\right)}{\operatorname{ess} \mathbb{P u p}^{\mathbb{P}}} y_{t_{1}}^{\mathbb{P}^{\prime}}\left(t_{2}, Y_{t_{2}}\right), \mathbb{P}-\text { a.s. }
$$

(ii) To prove the reverse inequality in representation (3.1), we use standard linearization techniques. Fix $\mathbb{P} \in \mathcal{P}$, for every $\mathbb{P}^{\prime} \in \mathcal{P}\left(t_{1}^{+}, \mathbb{P}\right)$, denote $\delta Y:=Y-y^{\mathbb{P}^{\prime}}\left(t_{2}, Y_{t_{2}}\right)$ and $\delta Z:=Z-z^{\mathbb{P}^{\prime}}\left(t_{2}, Y_{t_{2}}\right)$. By Assumption 2.1(iii), there exist bounded processes $\lambda, \eta, \gamma, \beta$, which are respectively $\mathbb{R}, \mathbb{R}^{d}, \mathbb{R}^{d}$ and $\mathbb{R}$-valued, such that, $\mathbb{P}^{\prime}-$ a.s.

$$
\delta Y_{t}=\int_{t}^{t_{2}}\left(\lambda_{s} \delta Y_{s}+\eta_{s} \cdot \widehat{a}_{s}^{\frac{1}{2}} \delta Z_{s}\right) d s+\int_{t}^{t_{2}}\left(\gamma_{s} \delta Y_{s}+\beta_{s} \delta Z_{s}\right) \cdot d \overleftarrow{W}_{s}-\int_{t}^{t_{2}} \delta Z_{s} \cdot d B_{s}+K_{t_{2}}-K_{t}
$$

Define

$$
M_{t}:=\exp \left(\int_{0}^{t} \eta_{s} \cdot \widehat{a}_{s}^{-1 / 2} d B_{s}+\int_{0}^{t} \lambda_{s} d s-\frac{1}{2} \int_{0}^{t}\left\|\eta_{s}\right\|^{2} d s\right), t_{1} \leq t \leq t_{2}, \mathbb{P}^{\prime}-a . s .
$$

By integration by parts, we have

$$
d\left(M_{t} \delta Y_{t}\right)=M_{t}\left(\delta Z_{t}+\delta Y_{t} \eta_{t} \widehat{a}_{t}^{-1 / 2}\right) \cdot d B_{t}-M_{t} \beta_{t} \delta Z_{t} \cdot d \overleftarrow{W}_{t}-M_{t} d K_{t}
$$

We deduce

$$
\mathbb{E}^{\mathbb{P}}\left[\delta Y_{t_{1}}\right]=\mathbb{E}^{\mathbb{P}^{\prime}}\left[M_{t_{1}}^{-1} \int_{t_{1}}^{t_{2}} M_{t} d K_{t}\right] \leq \mathbb{E}^{\mathbb{P}^{\prime}}\left[\sup _{t_{1} \leq t \leq t_{2}}\left(M_{t_{1}}^{-1} M_{t}\right)\left(K_{t_{2}}-K_{t_{1}}\right)\right]
$$

where we used the fact that $K^{\mathbb{P}^{\prime}}$ is non-decreasing and that since $\delta Y_{t_{1}}$ is $\mathcal{F}_{t_{1}^{+}}$-measurable, its expectation is the same under $\mathbb{P}$ and $\mathbb{P}^{\prime}$. By the boundedness of $\lambda, \eta, \gamma, \beta$, for every $p \geq 1$ we have,

$$
\mathbb{E}^{\mathbb{P}^{\prime}}\left[\sup _{t_{1} \leq t \leq t_{2}}\left(M_{t_{1}}^{-1} M_{t}\right)^{p}+\sup _{t_{1} \leq t \leq t_{2}}\left(M_{t_{1}} M_{t}^{-1}\right)^{p}\right] \leq C_{p}, t_{1} \leq t \leq t_{2}, \mathbb{P}^{\prime}-\text { a.s. }
$$

Then it follows from the Hölder inequality that

$$
\begin{aligned}
\mathbb{E}^{\mathbb{P}}\left[Y_{t_{1}}-y_{t_{1}}^{\mathbb{P}^{\prime}}\left(t_{2}, Y_{t_{2}}\right)\right] & \leq\left(\mathbb{E}^{\mathbb{P}^{\prime}}\left[\sup _{t_{1} \leq t \leq t_{2}}\left(M_{t_{1}}^{-1} M_{t}\right)^{3}\right]\right)^{1 / 3}\left(\mathbb{E}^{\mathbb{P}^{\prime}}\left[\left(K_{t_{2}}-K_{t_{1}}\right)^{3 / 2}\right]\right)^{2 / 3} \\
& \leq C\left(\mathbb{E}^{\mathbb{P}^{\prime}}\left[K_{t_{2}}-K_{t_{1}}\right] \mathbb{E}^{\mathbb{P}^{\prime}}\left[\left(K_{t_{2}}-K_{t_{1}}\right)^{2}\right]\right)^{1 / 3}
\end{aligned}
$$

From the definition of $K$, we have

$$
\sup _{\mathbb{P}^{\prime} \in \mathcal{P}\left(t_{1}^{+}, \mathbb{P}\right)} \mathbb{E}^{\mathbb{P}^{\prime}}\left[\left(K_{t_{2}}-K_{t_{1}}\right)^{2}\right] \leq C\left(\|Y\|_{\mathbb{D}^{2}}^{2}+\|Z\|_{\mathbb{H}^{2}}^{2}+\left(\phi^{2, \varepsilon}\right)^{\frac{2}{2+\varepsilon}}+\left(\psi^{2, \varepsilon}\right)^{\frac{2}{2+\varepsilon}}\right)<+\infty .
$$


Then, by taking the infimum in $\mathcal{P}\left(t_{1}^{+}, \mathbb{P}\right)$ in the last inequality and using (3.4) and the result of Lemma 3.1 , we obtain

$$
\inf _{\mathbb{P}^{\prime} \in \mathcal{P}\left(t_{1}^{+}, \mathbb{P}\right)} \mathbb{E}^{\mathbb{P}}\left[Y_{t_{1}}-y_{t_{1}}^{\mathbb{P}^{\prime}}\left(t_{2}, Y_{t_{2}}\right)\right] \leq 0
$$

But we clearly have

$$
0 \geq \inf _{\mathbb{P}^{\prime} \in \mathcal{P}\left(t_{1}^{+}, \mathbb{P}\right)} \mathbb{E}^{\mathbb{P}}\left[Y_{t_{1}}-y_{t_{1}}^{\mathbb{P}^{\prime}}\left(t_{2}, Y_{t_{2}}\right)\right] \geq \mathbb{E}^{\mathbb{P}}\left[Y_{t_{1}}-{\operatorname{ess} \sup ^{\prime}}_{\mathbb{P}^{\prime} \in \mathcal{P}\left(t_{1}^{+}, \mathbb{P}\right)} y_{t_{1}}^{\mathbb{P}^{\prime}}\left(t_{2}, Y_{t_{2}}\right)\right]
$$

Since the quantity under the expectation is positive $\mathbb{P}-$ a.s. by Step 1 , we deduce that it is actually equal to $0, \mathbb{P}-$ a.s., which is the desired result.

As an immediate consequence of the representation formula (3.1) together with the comparison principle for BDSDEs, we have the following comparison principle for 2BDSDEs.

Theorem 3.2. Let $(Y, Z)$ and $\left(Y^{\prime}, Z^{\prime}\right)$ be the solutions of 2 BDSDEs with terminal conditions $\xi$ and $\xi^{\prime}$ and generators $\widehat{F}$ and $\widehat{F}^{\prime}$ respectively, and let $\left(y^{\mathbb{P}}, z^{\mathbb{P}}\right)$ and $\left(y^{\prime \mathbb{P}}, z^{\prime \mathbb{P}}\right)$ the solutions of the associated BDSDEs. Assume that they both verify Assumptions 2.1 and 2.2, and that we have $\mathcal{P}-$ q.s., $\xi \leq \xi^{\prime}, \widehat{F}\left(y_{t}^{\prime \mathbb{P}}, z_{t}^{\prime \mathbb{P}}\right) \leq \widehat{F}^{\prime}\left(y_{t}^{\prime \mathbb{P}}, z_{t}^{\prime \mathbb{P}}\right)$. Then $Y \leq Y^{\prime}, \mathcal{P}-$ q.s.

\subsection{A priori estimates}

In this section, we show some a priori estimates which will be not only useful in the sequel, but also ensure the uniqueness of a solution to a 2DBSDE in $\mathbb{D}^{2} \times \mathbb{H}^{2}$. We start with a reminder of $L^{p}$ estimates for solutions of BDSDEs which were proved in [52] (see Theorem 4.1 p.217).

Theorem 3.3. Let Assumptions 2.1, 2.2 and 2.3 hold and assume that $\xi \in L^{2+\varepsilon}$ for some $\varepsilon>0$. We have

$$
\mathbb{E}^{\mathbb{P}}\left[\sup _{0 \leq t \leq T}\left|y_{t}^{\mathbb{P}}\right|^{2+\varepsilon}+\left(\int_{0}^{T}\left\|\widehat{a}_{s}^{1 / 2} z_{s}^{\mathbb{P}}\right\|^{2} d s\right)^{\frac{2+\varepsilon}{2}}\right] \leq C \mathbb{E}^{\mathbb{P}}\left[|\xi|^{2+\varepsilon}+\int_{0}^{T}\left(\left|\widehat{F}_{t}^{0}\right|^{2+\varepsilon}+\left\|g_{t}^{0}\right\|^{2+\varepsilon}\right) d t\right] .
$$

The main result of this section is then

Theorem 3.4. Let Assumptions 2.1, 2.2 and 2.3 hold.

(i) Assume $\xi \in \mathbb{L}^{2} \cap L^{2+\varepsilon}$ and that $(Y, Z, K)$ is a solution to the $2 \operatorname{BDSDE}(2.1)$. Then, for any $\varepsilon^{\prime} \in(0, \varepsilon)$, there exist a constant $C$ such that

$$
\|Y\|_{\mathbb{D}^{2+\varepsilon^{\prime}}}^{2+\varepsilon^{\prime}}+\|Z\|_{\mathbb{H}^{2+\varepsilon^{\prime}}}^{2++\varepsilon^{\prime}}+\sup _{\mathbb{P} \in \mathcal{P}} \mathbb{E}^{\mathbb{P}}\left[\left|K_{T}\right|^{2+\varepsilon^{\prime}}\right] \leq C\left(\|\xi\|_{\mathbb{L}^{2+\varepsilon^{\prime}}}^{2+\varepsilon^{\prime}}+\|\xi\|_{L^{2+\varepsilon}}^{\frac{2+\varepsilon^{\prime}}{2+\varepsilon}}+\left(\phi^{2, \varepsilon}\right)^{\frac{2+\varepsilon^{\prime}}{2+\varepsilon}}+\left(\psi^{2, \varepsilon}\right)^{\frac{2+\varepsilon^{\prime}}{2+\varepsilon}}\right) .
$$

(ii) Assume $\xi^{i} \in \mathbb{L}^{2} \cap L^{2+\varepsilon}$ and that $\left(Y^{i}, Z^{i}, K^{i}\right)$ is a solution to the $2 \operatorname{BDSDE}(2.1), i=1,2$. Denote $\delta \xi:=$ $\xi^{1}-\xi^{2}, \delta Y:=Y^{1}-Y^{2}, \delta Z:=Z^{1}-Z^{2}$, and $\delta K:=K^{1}-K^{2}$. Then, there exist a constant $C$ such that

$$
\|\delta Y\|_{\mathbb{D}^{2}} \leq C\|\delta \xi\|_{\mathbb{L}^{2}},\|\delta Z\|_{\mathbb{H}^{2}}^{2}+\|\delta K\|_{\mathbb{H}^{2}}^{2} \leq C\|\delta \xi\|_{\mathbb{L}^{2}} \sum_{i=1}^{2}\left(\left\|\xi^{i}\right\|_{\mathbb{L}^{2}}^{2}+\left\|\xi^{i}\right\|_{L^{2+\varepsilon}}^{\frac{2}{2+\varepsilon}}+\left(\phi^{2, \varepsilon}\right)^{\frac{2}{2+\varepsilon}}+\left(\psi^{2, \varepsilon}\right)^{\frac{2}{2+\varepsilon}}\right) .
$$


Proof. (i) For every $\mathbb{P} \in \mathcal{P}$ and $\mathbb{P}^{\prime} \in \mathcal{P}\left(t^{+}, \mathbb{P}\right)$ we have, using Theorem 3.1 and the usual linearization procedure, that for some bounded processes $\alpha$ and $\beta$ and any $\varepsilon^{\prime} \in(0, \varepsilon)$

$$
\begin{aligned}
& \mathbb{E}^{\mathbb{P}}\left[\sup _{0 \leq t \leq T}\left|Y_{t}\right|^{2+\varepsilon^{\prime}}\right]=\mathbb{E}^{\mathbb{P}}\left[\sup _{0 \leq t \leq T}\left(\operatorname{ess~sup}_{\mathbb{P}^{\prime} \in \mathcal{P}\left(t^{+}, \mathbb{P}\right)}^{\mathbb{P}}\left|y_{t}^{\mathbb{P}^{\prime}}\right|\right)^{2+\varepsilon^{\prime}}\right] \\
\leq & \mathbb{E}^{\mathbb{P}}\left[\operatorname { s u p } _ { 0 \leq t \leq T } \left(\mathbb { E } _ { t } ^ { \mathcal { P } , \mathbb { P } } \left[|\xi|+\int_{t}^{T}\left|\widehat{F}_{s}^{0}\right| d s+C \int_{t}^{T}\left(\left|y_{s}^{\mathbb{P}^{\prime}}\right|+\left\|\widehat{a}_{s}^{1 / 2} z_{s}^{\mathbb{P}^{\prime}}\right\|\right) d s+\left|\int_{t}^{T} g_{s}^{0} \cdot d \overleftarrow{W}_{s}\right|\right.\right.\right. \\
& \left.\left.\left.+\left|\int_{t}^{T} \alpha_{s} y_{s}^{\mathbb{P}^{\prime}} \cdot d \overleftarrow{W}_{s}\right|+\left|\int_{t}^{T} \beta_{s} z_{s}^{\mathbb{P}^{\prime}} \cdot d \overleftarrow{W}_{s}\right|+\left|\int_{t}^{T} z_{s}^{\mathbb{P}^{\prime}} \cdot d B_{s}\right|\right]\right)^{2+\varepsilon^{\prime}}\right] \\
\leq & C\left(\mathbb{E}^{\mathbb{P}}\left[\sup _{0 \leq t \leq T} \mathbb{E}_{t}^{\mathcal{P}, \mathbb{P}}\left[\left.|\xi|\right|^{2+\varepsilon^{\prime}}\right]+\mathbb{E}^{\mathbb{P}}\left[\sup _{0 \leq t \leq T}\left(\mathbb{E}_{t}^{\mathcal{P}, \mathbb{P}}\left[\int_{0}^{T}\left|\widehat{F}_{s}^{0}\right| d s\right]\right)^{2+\varepsilon^{\prime}}\right]\right)\right. \\
& +C\left(\mathbb{E}^{\mathbb{P}}\left[\sup _{0 \leq t \leq T}\left(\mathbb{E}_{t}^{\mathcal{P}, \mathbb{P}}\left[\int_{0}^{T}\left|y_{s}^{\mathbb{P}^{\prime}}\right| d s\right]\right)^{2+\varepsilon^{\prime}}\right]+\mathbb{E}^{\mathbb{P}}\left[\sup _{0 \leq t \leq T}\left(\mathbb{E}_{t}^{\mathcal{P}, \mathbb{P}}\left[\int_{0}^{T}\left\|\widehat{a}_{s}^{1 / 2} z_{s}^{\mathbb{P}^{\prime}}\right\| d s\right]\right)^{2+\varepsilon^{\prime}}\right]\right) \\
& +C\left(\mathbb{E}^{\mathbb{P}}\left[\sup _{0 \leq t \leq T}\left(\mathbb{E}_{t}^{\mathcal{P}, \mathbb{P}}\left[\left|\int_{t}^{T} g_{s}^{0} \cdot d \overleftarrow{W}_{s}\right|\right]\right)^{2+\varepsilon^{\prime}}\right]+\mathbb{E}^{\mathbb{P}}\left[\sup _{0 \leq t \leq T}\left(\mathbb{E}_{t}^{\mathcal{P}, \mathbb{P}}\left[\left|\int_{t}^{T} z_{s}^{\mathbb{P}^{\prime}} \cdot d B_{s}\right|\right]\right)^{2+\varepsilon^{\prime}}\right]\right) \\
& +C \mathbb{E}^{\mathbb{P}}\left[\sup _{0 \leq t \leq T}\left(\mathbb{E}_{t}^{\mathcal{P}, \mathbb{P}}\left[\left|\int_{t}^{T} y_{s}^{\mathbb{P}^{\prime}} \alpha_{s} \cdot d \overleftarrow{W}_{s}\right|\right]\right)^{2+\varepsilon^{\prime}}+\sup _{0 \leq t \leq T}\left(\mathbb{E}_{t}^{\mathcal{P}, \mathbb{P}}\left[\left|\int_{t}^{T} z_{s}^{\mathbb{P}^{\prime}} \beta_{s} \cdot d \overleftarrow{W}_{s}\right|\right]\right)^{2+\varepsilon^{\prime}}\right] .
\end{aligned}
$$

Now we remind the reader that by $([62],[56])$, for any random variable $A$, we have

$$
\sup _{\mathbb{P} \in \mathcal{P}} \mathbb{E}^{\mathbb{P}}\left[\sup _{0 \leq t \leq T} \mathbb{E}_{t}^{\mathcal{P}, \mathbb{P}}[|A|]^{2+\varepsilon^{\prime}}\right] \leq C \sup _{\mathbb{P} \in \mathcal{P}}\left(\mathbb{E}^{\mathbb{P}}\left[|A|^{2+\epsilon}\right]\right)^{\frac{2+\varepsilon^{\prime}}{2+\epsilon}}
$$

We therefore deduce with BDG inequalities that (remember that $\alpha$ and $\beta$ are bounded)

$$
\begin{aligned}
\mathbb{E}^{\mathbb{P}}\left[\sup _{0 \leq t \leq T}\left|Y_{t}\right|^{2+\varepsilon^{\prime}}\right] \leq & C\left(\|\xi\|_{\mathbb{L}^{2+\varepsilon^{\prime}}}^{2+\varepsilon^{\prime}}+\left(\phi^{2, \varepsilon}\right)^{\frac{2+\varepsilon^{\prime}}{2+\varepsilon}}+\left(\psi^{2, \varepsilon}\right)^{\frac{2+\varepsilon^{\prime}}{2+\varepsilon}}\right) \\
& +C \sup _{\mathbb{P} \in \mathcal{P}} \mathbb{E}^{\mathbb{P}}\left[\sup _{0 \leq t \leq T}\left|y_{t}^{\mathbb{P}}\right|^{2+\varepsilon}+\left(\int_{0}^{T}\left\|\widehat{a}_{s}^{1 / 2} z_{s}^{\mathbb{P}}\right\|^{2} d s\right)^{\frac{2+\varepsilon}{2}}\right]^{\frac{2+\varepsilon^{\prime}}{2+\varepsilon}} .
\end{aligned}
$$

Finally, we obtain by Theorem 3.3

$$
\|Y\|_{\mathbb{D}^{2+\varepsilon^{\prime}}}^{2+\varepsilon^{\prime}} \leq C\left(\|\xi\|_{\mathbb{L}^{2+\varepsilon^{\prime}}}^{2+\varepsilon^{\prime}}+\|\xi\|_{L^{2+\varepsilon}}^{2+\varepsilon^{\prime}}+\left(\phi^{2, \varepsilon}\right)^{\frac{2+\varepsilon^{\prime}}{2+\varepsilon}}+\left(\psi^{2, \varepsilon}\right)^{\frac{2+\varepsilon^{\prime}}{2+\varepsilon}}\right)
$$

When it comes to the estimate for $Z$, we apply Itô's formula to $|Y|^{2}$ under each $\mathbb{P} \in \mathcal{P}$ and from the Lipschitz Assumption 2.1(iii) we have, using BDG inequality and our assumptions on $g$ and $\widehat{F}$ 


$$
\begin{aligned}
\mathbb{E}^{\mathbb{P}}\left[\left(\int_{0}^{T}\left\|\widehat{a}_{s}^{1 / 2} Z_{s}\right\|^{2} d s\right)^{\frac{2+\varepsilon^{\prime}}{2}}\right] \leq & C \mathbb{E}^{\mathbb{P}}\left[|\xi|^{2+\varepsilon^{\prime}}+\left(\int_{0}^{T}\left|Y_{s}\right|\left(\left|\widehat{F}_{s}^{0}\right|+\left|Y_{s}\right|+\left\|\widehat{a}_{s}^{1 / 2} Z_{s}\right\|\right) d s\right)^{\frac{2+\varepsilon^{\prime}}{2}}\right] \\
& +C \mathbb{E}^{\mathbb{P}}\left[\left(\int_{0}^{T} Y_{s}^{2}\left\|g_{s}\left(Y_{s}, Z_{s}\right)\right\|^{2} d s\right)^{\frac{2+\varepsilon^{\prime}}{4}}+\left(\int_{0}^{T} Y_{s}^{2}\left\|\widehat{a}_{s}^{1 / 2} Z_{s}\right\|^{2} d s\right)^{\frac{2+\varepsilon^{\prime}}{4}}\right] \\
& +C \mathbb{E}^{\mathbb{P}}\left[\left(\int_{0}^{T}\left\|g_{s}\left(Y_{s}, Z_{s}\right)\right\|^{2} d s\right)^{\frac{2+\varepsilon^{\prime}}{2}}+\int_{0}^{T}\left|Y_{s}\right|^{\frac{2+\varepsilon^{\prime}}{2}} d K_{s}\right] \\
\leq & C \nu^{-1} \mathbb{E}^{\mathbb{P}}\left[|\xi|^{2+\varepsilon^{\prime}}+\sup _{0 \leq s \leq T}\left|Y_{s}\right|^{2+\varepsilon^{\prime}}+\int_{0}^{T}\left|\widehat{F}_{s}^{0}\right|^{2+\varepsilon^{\prime}} d s+\int_{0}^{T}\left\|g_{s}^{0}\right\|^{2+\varepsilon^{\prime}} d s\right] \\
& +\nu \mathbb{E}^{\mathbb{P}}\left[\left(\int_{0}^{T}\left\|\widehat{a}_{s}^{1 / 2} Z_{s}\right\|^{2} d s\right)^{\frac{2+\varepsilon^{\prime}}{2}}+\left|K_{T}\right|^{2+\varepsilon^{\prime}}+\alpha^{\frac{2+\varepsilon^{\prime}}{2}}\left(\int_{0}^{T}\left\|Z_{s}\right\|^{2} d s\right)^{\frac{2+\varepsilon^{\prime}}{2}}\right],
\end{aligned}
$$

for any $\nu \in(0,1]$. But by the definition of $K_{T}$, it is clear that

$$
\mathbb{E}^{\mathbb{P}}\left[\left|K_{T}\right|^{2+\varepsilon^{\prime}}\right] \leq C_{0} \mathbb{E}^{\mathbb{P}}\left[|\xi|^{2+\varepsilon^{\prime}}+\sup _{0 \leq s \leq T}\left|Y_{s}\right|^{2+\varepsilon^{\prime}}+\left(\int_{0}^{T}\left\|\widehat{a}_{s}^{1 / 2} Z_{s}\right\|^{2} d s\right)^{\frac{2+\varepsilon^{\prime}}{2}}+\int_{0}^{T}\left(\left|\widehat{F}_{s}^{0}\right|^{2+\varepsilon^{\prime}}+\left\|g_{s}^{0}\right\|^{2+\varepsilon^{\prime}}\right) d s\right],
$$

for some constant $C_{0}$ independent of $\varepsilon$.

Then, using in particular Assumption 2.1(iv)

$$
\begin{aligned}
\mathbb{E}^{\mathbb{P}}\left[\left(\int_{0}^{T}\left\|\widehat{a}_{s}^{1 / 2} Z_{s}\right\|^{2} d s\right)^{\frac{2+\varepsilon^{\prime}}{2}}\right] \leq & C \nu^{-1} \mathbb{E}^{\mathbb{P}}\left[|\xi|^{2+\varepsilon^{\prime}}+\sup _{0 \leq s \leq T}\left|Y_{s}\right|^{2+\varepsilon^{\prime}}+\int_{0}^{T}\left|\widehat{F}_{s}^{0}\right|^{2+\varepsilon^{\prime}} d s+\int_{0}^{T}\left\|g_{s}^{0}\right\|^{2+\varepsilon^{\prime}} d s\right] \\
& +\left(\nu+C_{0} \nu+(1-\lambda)^{\frac{2+\varepsilon^{\prime}}{2}}\right) \mathbb{E}^{\mathbb{P}}\left[\left(\int_{0}^{T}\left\|\widehat{a}_{s}^{1 / 2} Z_{s}\right\|^{2} d s\right)^{\frac{2+\varepsilon^{\prime}}{2}}\right] .
\end{aligned}
$$

Choosing $\nu$ small enough, this implies the desired result by (3.5). Finally, the estimate for the $K$ follows directly from (3.6).

(ii) First of all, we can follow the same arguments as in (i) above to obtain the existence of a constant $C$, depending only on $T$ and the Lipschitz constant of $\widehat{F}$ and $g$ such that for all $\mathbb{P} \in \mathcal{P}$

$$
\mathbb{E}^{\mathbb{P}}\left[\sup _{0 \leq t \leq T}\left|\delta Y_{t}\right|^{2}\right]=\mathbb{E}^{\mathbb{P}}\left[\sup _{0 \leq t \leq T}\left(\operatorname{ess~sup}_{\mathbb{P}^{\prime} \in \mathcal{P}\left(t^{+}, \mathbb{P}\right)}^{\mathbb{P}}\left|\delta y_{t}^{\mathbb{P}^{\prime}}\right|\right)^{2}\right] \leq C\left(\|\delta \xi\|_{\mathbb{L}^{2}}^{2}+\|\delta \xi\|_{\mathbb{L}^{2+\varepsilon}}^{\frac{2}{2+\varepsilon}}\right)
$$

Applying Itô formula to $|\delta Y|^{2}$, under each $\mathbb{P} \in \mathcal{P}$, leads to

$$
\begin{aligned}
\mathbb{E}^{\mathbb{P}}\left[\int_{0}^{T}\left\|\widehat{a}_{s}^{1 / 2} \delta Z_{s}\right\|^{2} d s\right] & \leq C \mathbb{E}^{\mathbb{P}}\left[|\delta \xi|^{2}+\int_{0}^{T}\left|\delta Y_{s}\right|\left(\left|\delta Y_{s}\right|+\left\|\widehat{a}_{s}^{1 / 2} \delta Z_{s}\right\|\right) d s+\int_{0}^{T}\left|\delta Y_{s}\right| d\left|\delta K_{s}\right|+\int_{0}^{T}\left|\delta Y_{s}\right|^{2} d s\right] \\
& \leq C \mathbb{E}^{\mathbb{P}}\left[|\delta \xi|^{2}+\sup _{0 \leq s \leq T}\left|\delta Y_{s}\right|^{2}+\sup _{0 \leq s \leq T}\left|\delta Y_{s}\right|^{2}\left[K_{T}^{1}+K_{T}^{2}\right]+\frac{1}{2} \int_{0}^{T}\left\|\widehat{a}_{s}^{1 / 2} \delta Z_{s}\right\|^{2} d s\right] .
\end{aligned}
$$


The estimate for $\delta Z$ is now obvious from the above inequality and the estimates of (i). Finally the estimate for the difference of the increasing processes is obvious by definition.

\section{Existence by a pathwise construction of the solution}

As we have shown in Theorem 3.1, if a solution to the 2BDSDE (2.1) exists, it necessarily can be represented as a supremum of solutions to standard BDSDEs. However, since we are working under a family of nondominated probability measures, we cannot use the classical technics of BSDEs to construct such a solution. We will therefore follow the original approach of Soner, Touzi and Zhang [64], who overcame this problem by constructing the solution pathwise, using the so-salled regular conditional probability distribution.

\subsection{Notations related to shifted spaces}

For any $0 \leq t \leq T$, we denote by $\Omega^{B, t}:=\left\{\omega \in C\left([t, T], \mathbb{R}^{d}\right), \omega(t)=0\right\}$ the shifted canonical space, $B^{t}$ the shifted canonical process, $\mathbb{P}_{0}^{B, t}$ the shifted Wiener measure and $\mathcal{F}^{B, t}$ the shifted raw filtration generated by $B^{t}$. The pathwise density of its quadratic variation is denoted by $\widehat{a}^{t}$. We then let $\Omega^{t}:=\Omega^{B, t} \times \Omega^{W}$, and exactly as in Section 2, we can define the set $\overline{\mathcal{P}}_{S}^{t}$, by restricting the corresponding measures to the shifted space $\Omega^{t}$.

Next, for any $0 \leq s \leq t \leq T$ and $\omega \in \Omega^{s}$, we define the shifted path $\omega^{t}:=\left(\omega^{B, t}, \omega^{W}\right) \in \Omega^{t}$ by

$$
\omega_{r}^{B, t}:=\omega_{r}^{B}-\omega_{t}^{B}, \forall r \in[t, T],
$$

and for $\omega^{B} \in \Omega^{B, s}, \tilde{\omega}^{B} \in \Omega^{B, t}$ we define the concatenated path by

$$
\left(\omega^{B} \otimes_{t} \tilde{\omega}^{B}\right)(r):=\omega_{r}^{B} \mathbf{1}_{[s, t)}(r)+\left(\omega_{t}^{B}+\tilde{\omega}_{r}^{B}\right) \mathbf{1}_{[t, T]}(r), \forall r \in[s, T] .
$$

Similarly, for any $\mathcal{F}_{T}^{s}-$ measurable random variable $\xi$ on $\Omega^{s}$, and for each $\left(\omega^{B}, \omega^{W}\right) \in \Omega^{s}$, we define the $\mathcal{F}_{T}^{t}$-measurable random variable $\xi^{t, \omega^{B}}$ on $\Omega^{t}$ by

$$
\xi^{t, \omega^{B}}\left(\tilde{\omega}^{B}, \omega^{W}\right):=\xi\left(\omega^{B} \otimes_{t} \tilde{\omega}^{B}, \omega^{W}\right), \forall\left(\tilde{\omega}^{B}, \omega^{W}\right) \in \Omega^{t} .
$$

The shifted generators that we consider are, for every $\left(s,\left(\tilde{\omega}^{B}, \omega^{W}\right)\right) \in[t, T] \times \Omega^{t}$

$$
\begin{aligned}
& \widehat{F}_{s}^{t, \omega^{B}}\left(\left(\tilde{\omega}^{B}, \omega^{W}\right), y, z\right):=F_{s}\left(\left(\omega^{B} \otimes_{t} \tilde{\omega}^{B}, \omega^{W}\right), y, z, \widehat{a}_{s}^{t}\left(\tilde{\omega}^{B}, \omega^{W}\right)\right), \\
& g_{s}^{t, \omega^{B}}\left(\left(\tilde{\omega}^{B}, \omega^{W}\right), y, z\right):=g_{s}\left(\left(\omega^{B} \otimes_{t} \tilde{\omega}^{B}, \omega^{W}\right), y, z\right) .
\end{aligned}
$$

Then note that since $F$ and $g$ are assumed to be uniformly continuous in $\omega$, then so are the maps $\left(\omega^{B}, \omega^{W}\right) \longmapsto$ $F_{s}\left(\left(\omega^{B} \otimes_{t} \cdot, \omega^{W}\right), \cdot\right)$ and $\left(\omega^{B}, \omega^{W}\right) \longmapsto g_{s}^{t, \omega^{B}}\left(\left(\cdot, \omega^{W}\right), \cdot\right)$. Notice that this implies that for any $\mathbb{P} \in \overline{\mathcal{P}}_{S}^{t}$

$$
\mathbb{E}^{\mathbb{P}}\left[\left(\int_{t}^{T}\left|\widehat{F}_{s}^{t, \omega^{B}}(0,0)\right|^{2} d s\right)\right]+\mathbb{E}^{\mathbb{P}}\left[\left(\int_{t}^{T}\left\|g_{s}^{t, \omega^{B}}(0,0)\right\|^{2} d s\right)\right]<+\infty,
$$

for some $\omega^{B} \in \Omega^{B}$ if and only if it holds for all $\omega^{B} \in \Omega^{B}$.

We also extend Definition 2.2 in the shifted spaces

Definition 4.1. $\mathcal{P}^{t}$ is the subset of $\overline{\mathcal{P}}_{S}^{t}$, consisting of measures $\mathbb{P}$ such that

$$
\begin{gathered}
\underline{a}_{\mathbb{P}} \leq \widehat{a}^{t} \leq \bar{a}_{\mathbb{P}}, d t \times d \mathbb{P}-\text { a.e. on }[t, T] \times \Omega^{t}, \text { for some } \underline{a}_{\mathbb{P}}, \bar{a}_{\mathbb{P}} \in \mathbb{S}_{d}^{>0}, \\
\mathbb{E}^{\mathbb{P}}\left[\left(\int_{t}^{T}\left|\widehat{F}_{s}^{t, \omega^{B}}(0,0)\right|^{2} d s\right)\right]+\mathbb{E}^{\mathbb{P}}\left[\left(\int_{t}^{T}\left\|g_{s}^{t, \omega^{B}}(0,0)\right\|^{2} d s\right)\right]<+\infty \text {, for all } \omega^{B} \in \Omega^{B} .
\end{gathered}
$$


Finally, by Stroock and Varadhan [67], for any $\mathbb{F}^{B}$-stopping time $\tau$, any probability measure $\mathbb{P}_{B}$ on $\left(\Omega^{B}, \mathcal{F}^{B}\right)$, and any $\omega^{B} \in \Omega^{B}$, there exists a regular conditional probability distribution (r.p.c.d. for short), $\mathbb{P}_{B, \tau\left(\omega^{B}\right)}^{B}$ with respect to the $\sigma$-field $\mathcal{F}_{\tau}^{B}$ (since it is countably generated). Such a measure verifies that for every integrable $\mathcal{F}_{T}^{B}$-measurable random variable $\xi$, we have for $\mathbb{P}^{B}-$ a.e. $\omega^{B}$

$$
\mathbb{E}^{\mathbb{P}^{B}}\left[\xi \mid \mathcal{F}_{\tau}^{B}\right]\left(\omega^{B}\right)=\mathbb{E}_{B, \tau\left(\omega^{B}\right)}^{\mathbb{P}^{\omega^{B}}}[\xi] .
$$

Furthermore, this r.c.p.d. naturally induces a probability measure $\mathbb{P}_{B}^{\tau\left(\omega^{B}\right), \omega^{B}}$ on $\left(\Omega^{B, \tau\left(\omega^{B}\right)}, \mathcal{F}_{T}^{B, \tau\left(\omega^{B}\right)}\right)$ such that

$$
\mathbb{E}^{\mathbb{P}_{B, \tau\left(\omega^{B}\right)}^{\omega^{B}}}[\xi]=\mathbb{E}^{\mathbb{P}_{B}^{\tau\left(\omega^{B}\right), \omega^{B}}}\left[\xi^{\tau\left(\omega^{B}\right), \omega^{B}}\right] .
$$

Notice that if we consider a stochastic kernel $\left\{\mathbb{P}_{B}\left(\omega^{W}\right), \omega^{W} \in \Omega^{W}\right\}$ on $\left(\Omega^{B}, \mathcal{F}_{T}^{B}\right)$, then for any $\omega^{B} \in \Omega^{B}$, $\left\{\mathbb{P}_{B}^{\tau\left(\omega^{B}\right), \omega^{B}}\left(\omega^{W}\right), \omega^{W} \in \Omega^{W}\right\}$ is a stochastic kernel on $\left(\Omega^{B, \tau\left(\omega^{B}\right)}, \mathcal{F}_{T}^{B, \tau\left(\omega^{B}\right)}\right)$.

Let us now consider a $\mathbb{G}$-stopping time $\tau$. Then, using the classical results of Stricker and Yor [66], we know that there is a $\mathbb{P}_{0}^{W}$ version of $\tau$ (still denoted $\tau$ for simplicity) such that the map $\omega^{B} \longmapsto \tau\left(\omega^{B}, \omega^{W}\right)$ defines a $\mathbb{F}^{B}$-stopping time for $\mathbb{P}_{0}^{W}$-a.e. $\omega^{W} \in \Omega^{W}$. Let again $\left\{\mathbb{P}_{B}\left(\omega^{W}\right), \omega^{W} \in \Omega^{W}\right\}$ be a stochastic kernel on $\left(\Omega^{B}, \mathcal{F}_{T}^{B}\right)$ and let us define the measure $\mathbb{P}$ on $(\Omega, \mathcal{F})$ by

$$
d \mathbb{P}\left(\omega^{B}, \omega^{W}\right)=d \mathbb{P}_{B}\left(\omega^{W} ; \omega^{B}\right) d \mathbb{P}_{0}^{W}\left(\omega^{W}\right) .
$$

We claim (and refer the reader to the proof of the more general result in Lemma 4.1) that we can write for $\mathbb{P}-$ a.e. $\omega \in \Omega$

$$
\begin{aligned}
\mathbb{E}^{\mathbb{P}}\left[\xi \mid \mathcal{G}_{\tau}\right]\left(\omega^{B}, \omega^{W}\right) & =\mathbb{E}^{\mathbb{P}_{0}^{W}}\left[\int_{\Omega^{B}} \xi^{\tau\left(\omega^{B}, \cdot\right), \omega^{B}}\left(\widetilde{\omega}^{B}\right) d \mathbb{P}_{B}^{\tau\left(\omega^{B}, \cdot\right), \omega^{B}}\left(\cdot ; \widetilde{\omega}^{B}\right) \mid \mathcal{F}_{T}^{W}\right]\left(\omega^{W}\right) \\
& =\mathbb{E}^{\mathbb{P}_{B}^{\tau\left(\omega^{B}, \omega^{W}\right), \omega^{B}}\left(\omega^{W}\right)}\left[\xi^{\tau\left(\omega^{B}, \omega^{W}\right), \omega^{B}}\left(\cdot, \omega^{W}\right)\right] .
\end{aligned}
$$

Moreover, by Lemma 4.1 in [64], we know that for any probability measure $\mathbb{P} \in \overline{\mathcal{P}}_{S}$ on $\left(\Omega, \mathcal{F}_{T}\right)$ such that $d \mathbb{P}\left(\omega^{B}, \omega^{W}\right)=d \mathbb{P}_{B}\left(\omega^{W} ; \omega^{B}\right) d \mathbb{P}_{0}^{W}\left(\omega^{W}\right)$, we have for $\mathbb{P}$ - a.e. $\omega \in \Omega$, for any $\mathbb{F}^{B}$-stopping time $\tau$ and for $d s \times d \mathbb{P}^{\tau\left(\omega^{B}\right), \omega^{B}}\left(\omega^{W}\right)$ - a.e. $\left(s, \tilde{\omega}^{B}\right) \in\left[\tau\left(\omega^{B}\right), T\right] \times \Omega^{B, \tau\left(\omega^{B}\right)}$

$$
\widehat{a}_{s}^{\tau\left(\omega^{B}\right), \omega^{B}}\left(\tilde{\omega}^{B}, \omega^{W}\right)=\widehat{a}_{s}^{\tau\left(\omega^{B}\right)}\left(\tilde{\omega}^{B}, \omega^{W}\right),
$$

which justifies the definition of the shifted generator $\widehat{F}^{t, \omega^{B}}$.

\subsection{Existence when $\xi$ is in $\mathrm{UC}_{b}(\Omega)$}

When $\xi$ is in $\mathrm{UC}_{b}(\Omega)$, we know that there exists a modulus of continuity function $\rho$ for $\xi, F$ and $g$ in $\omega$. Then, for any $0 \leq t \leq s \leq T,(y, z) \in \mathbb{R} \times \mathbb{R}^{d}$ and $\left(\omega^{B}, \omega^{\prime, B}\right) \in \Omega^{B} \times \Omega^{B}, \widetilde{\omega}^{B} \in \Omega^{B, t}$ and $\omega^{W} \in \Omega^{W}$,

$$
\begin{aligned}
\left|\xi^{t, \omega^{B}}\left(\widetilde{\omega}^{B}, \omega^{W}\right)-\xi^{t, \omega^{\prime, B}}\left(\widetilde{\omega}^{B}, \omega^{W}\right)\right| & \leq \rho\left(\left\|\omega^{B}-\omega^{\prime, B}\right\|_{t}\right), \\
\left|\widehat{F}_{s}^{t, \omega^{B}}\left(\left(\widetilde{\omega}^{B}, \omega^{W}\right), y, z\right)-\widehat{F}_{s}^{t, \omega^{\prime, B}}\left(\left(\widetilde{\omega}^{B}, \omega^{W}\right), y, z\right)\right| & \leq \rho\left(\left\|\omega^{B}-\omega^{\prime, B}\right\|_{t}\right), \\
\left|g_{s}^{t, \omega^{B}}\left(\left(\widetilde{\omega}^{B}, \omega^{W}\right), y, z\right)-g_{s}^{t, \omega^{\prime, B}}\left(\left(\widetilde{\omega}^{B}, \omega^{W}\right), y, z\right)\right| & \leq \rho\left(\left\|\omega^{B}-\omega^{\prime, B}\right\|_{t}\right) .
\end{aligned}
$$

Using this regularity and Assumption 2.1, it is easy to see that we have for all $\left(t, \omega^{B}\right) \in[0, T] \times \Omega^{B}$.

$$
\Lambda_{t}\left(\omega^{B}\right):=\sup _{\mathbb{P} \in \mathcal{P}^{t}}\left(\mathbb{E}^{\mathbb{P}}\left[\left|\xi^{t, \omega^{B}}\right|^{2}+\int_{t}^{T}\left|\widehat{F}_{s}^{t, \omega^{B}}(0,0)\right|^{2} d s+\int_{t}^{T}\left\|g_{s}^{t, \omega^{B}}(0,0)\right\|^{2} d s\right]\right)^{1 / 2}<+\infty .
$$


To prove existence, we define the following value process $V_{t}$ for every $\omega^{B}$

$$
V_{t}\left(\omega^{B}, \cdot\right):=\underset{\mathbb{P} \in \mathcal{P}^{t}}{\operatorname{ess} \sup _{W}} \mathbb{P}_{W}^{0} \mathcal{Y}_{t}^{\mathbb{P}, t, \omega^{B}}(T, \xi)(\cdot), \mathbb{P}_{W}^{0}-\text { a.s. },
$$

where, for any $\left(t_{1}, \omega^{B}\right) \in[0, T] \times \Omega^{B}, \mathbb{P} \in \mathcal{P}^{t_{1}}, t_{2} \in\left[t_{1}, T\right]$, and any $\mathcal{F}_{t_{2}}$-measurable $\eta \in \mathbb{L}^{2}(\mathbb{P})$, we denote $\mathcal{Y}_{t_{1}, t_{1}, \omega^{B}}^{\mathbb{P}}\left(t_{2}, \eta\right):=y_{t_{1}}^{\mathbb{P}, t_{1}, \omega^{B}}$, where $\left(y^{\mathbb{P}, t_{1}, \omega^{B}}, z^{\mathbb{P}, t_{1}, \omega^{B}}\right)$ is the solution of the following BDSDE on the shifted space $\Omega^{t_{1}}$ under $\mathbb{P}$,

$$
y_{s}^{\mathbb{P}, t_{1}, \omega^{B}}=\eta^{t_{1}, \omega^{B}}+\int_{s}^{t_{2}} \widehat{F}_{r}^{t_{1}, \omega^{B}}\left(y_{r}^{\mathbb{P}, t_{1}, \omega^{B}}, z_{r}^{\mathbb{P}, t_{1}, \omega^{B}}\right) d r-\int_{s}^{t_{2}} z_{r}^{\mathbb{P}, t_{1}, \omega^{B}} \cdot d B_{r}^{t_{1}}+\int_{s}^{t_{2}} g_{r}^{t_{1}, \omega^{B}}\left(y_{r}^{\mathbb{P}, t_{1}, \omega^{B}}, z_{r}^{\mathbb{P}, t_{1}, \omega^{B}}\right) \cdot d \overleftarrow{W}_{r}
$$

The following Lemma allows to give a link between BDSDEs on the shifted spaces. Its technical proof is postponed to the Appendix.

Lemma 4.1. Fix some $\mathbb{P} \in \overline{\mathcal{P}}_{S}$ such that $d \mathbb{P}\left(\omega^{B}, \omega^{W}\right):=d \mathbb{P}_{B}\left(\omega^{B} ; \omega^{W}\right) d \mathbb{P}_{0}^{W}\left(\omega^{W}\right)$. For $\mathbb{P}-$ a.e. $\omega \in \Omega$, the following equality holds

$$
y_{t}^{\mathbb{P}^{t, \omega^{B}}(\cdot) \otimes \mathbb{P}_{W}^{0}}\left(\omega^{W}\right)=y_{t}^{\mathbb{P}}\left(\omega^{B}, \omega^{W}\right), t \in[0, T],
$$

where $d\left(\mathbb{P}_{B}^{t, \omega^{B}}(\cdot) \otimes \mathbb{P}_{W}^{0}\right)\left(\omega^{B}, \omega^{W}\right):=d \mathbb{P}_{B}^{t, \omega^{B}}\left(\omega^{B} ; \omega^{W}\right) d \mathbb{P}_{0}^{W}\left(\omega^{W}\right)$.

We point out that for classical 2BSDEs, Soner, Touzi and Zhang have proved in Lemma 4.6 of [64] a regularity result for the value process, precisely the uniform continuity with respect to the trajectory $\omega^{B}$ and this is crucial to prove their dynamic programming principle (Proposition 4.7 in [64]). Since in our context, the value process $V$ defined in (4.2) is a random field depending on two source of randomness, we prove the following regularity result which is weaker than Lemma 4.6 of [64]. Once again, we cannot obtain the same regularity in the context of doubly stochastic 2BSDEs because we cannot have path-wise estimates for their solutions.

Lemma 4.2. We have for every $\left(\omega^{B, 1}, \omega^{B, 2}\right) \in \Omega^{B} \times \Omega^{B}$

$$
\mathbb{E}^{\mathbb{P}_{W}^{0}}\left[\left(V_{t}\left(\omega^{B, 1} \cdot\right)-V_{t}\left(\omega^{B, 2} \cdot\right)\right)^{2}\right] \leq \rho^{2}\left(\left\|\omega^{B, 1}-\omega^{B, 2}\right\|_{t}\right) .
$$

In particular, this implies that the map $\omega^{B} \longmapsto V_{t}\left(\omega^{B}, \cdot\right)$ is uniformly continuous in probability (with respect to $\left.\mathbb{P}_{W}^{0}\right)$, which implies that there is a $\mathbb{P}_{W}^{0}$-version, which we still denote $V$ for simplicity, which is jointly measurable in $\left(\omega^{B}, \omega^{W}\right)$, and more precisely, such that $V_{t}$ is $\mathcal{F}_{t}-$ measurable or even $\mathcal{F}_{t}^{B} \otimes \mathcal{F}_{t, T}^{o, W}-$ measurable.

Proof. The estimate is an easy consequence of classical a priori estimates for BDSDEs, using in particular the uniform continuity in $\omega$ of both $F, g$ and $\xi$. The reasoning is quite similar to the one we used in the proof of Theorem 3.4, so that we omit it. As for the existence of measurable version, this is a classical result using the fact that the topology of convergence in probability is metrizable (see for instance Dellacherie and Meyer [21], chapter IV, Theorem 30, or the proof of Corollary A.3 in [19]).

We then have the following joint measurability result

Lemma 4.3. The map $\left(t, \omega^{B}, \omega^{W}\right) \longmapsto V_{t}\left(\omega^{B}, \omega^{W}\right)$ is $\mathcal{B}([0, T]) \otimes \mathcal{F}_{t}$-measurable.

Proof. First of all, we claim that the family $\left\{\mathcal{Y}_{t}^{\mathbb{P}, t, \omega^{B}}(T, \xi), \mathbb{P} \in \mathcal{P}^{t}\right\}$ is upward directed. Indeed, this can be proved exactly as in Step (iii) of the proof of Theorem 4.3 of [65]. As a consequence, we know that there is a sequence $\left(\mathbb{P}^{n}\right)_{n \geq 0} \subset \mathcal{P}^{t}$ such that for $\mathbb{P}_{0}^{W}-$ a.e. $\omega^{W} \in \Omega^{W}$

$$
V_{t}\left(\omega^{B}, \omega^{W}\right)=\sup _{n \geq 0} \mathcal{Y}_{t}^{\mathbb{P}^{n}, t \omega^{B}}\left(\omega^{W}\right)
$$


Now arguing exactly as in Step (i) of the proof of Theorem 2.1 in [58], using in particular the fact that we can always mimic the construction in Section 2.5.2 of [58] to obtain that the map $\left(t, \mathbb{P}, \omega^{B}, \omega^{W}\right) \longmapsto \mathcal{Y}_{t}^{\mathbb{P}, t \omega^{B}}\left(\omega^{W}\right)$ is Borel measurable, we deduce that $\left(t, \omega^{B}, \omega^{W}\right) \longmapsto V_{t}\left(\omega^{B}, \omega^{W}\right)$ is $\mathcal{B}([0, T]) \otimes \mathcal{F}_{T}^{B} \otimes \mathcal{F}_{0, T}^{o, W}$-universally measurable. But then it suffices to use the result of Lemma 4.2 to conclude.

Now, we present the main result concerning the dynamic programming principle in our context. We follow the approach of Possamaï, Tan and Zhou [58], where they proved existence result for 2BSDEs with only measurable parameters. Their proof is based on dynamic programming principle without regularity on the terminal condition and the generator, which is itself strongly inspired by the classical results recalled, for instance, in the papers $[26,27]$. We therefore omit the proof.

Theorem 4.1. Under the Assumptions 2.1, 2.2 and for $\xi \in \mathrm{UC}_{b}(\Omega)$, we have for all $0 \leq t_{1} \leq t_{2} \leq T$

$$
V_{t_{1}}\left(\omega^{B}, \omega^{W}\right)=\underset{\mathbb{P} \in \mathcal{P}^{t_{1}}}{\operatorname{ess} \operatorname{Pu}^{\mathbb{P}}} \mathcal{Y}_{t_{1}}^{\mathbb{P}, t_{1}, \omega^{B}}\left(t_{2}, V_{t_{2}}^{t_{1}, \omega^{B}}\left(\cdot, \omega^{W}\right)\right), \mathbb{P}-\text { a.e. } \omega \in \Omega .
$$

Next, we introduce the right limit of the $\mathrm{V}$ which is clearly $\mathcal{F}_{t^{+}}-$measurable

$$
V_{t}^{+}:=\varlimsup_{r \in \mathbb{Q} \cap(t, T], r \downarrow t} V_{r} .
$$

We have the following regularity result, whose proof is postponed until the appendix.

Lemma 4.4. Under the Assumptions 2.1, 2.2, we have

$$
V_{t}^{+}=\lim _{r \in \mathbb{Q} \cap(t, T], r \downarrow t} V_{r}, \mathcal{P}-q . s .
$$

and thus $V^{+}$is càdlàg $\mathcal{P}-$ q.s.

Thanks to the dynamic programming principle for $V$, as well as the just proved regularity of $V^{+}$, we can now show that $V^{+}$is actually a semi-martingale under any $\mathbb{P} \in \mathcal{P}$ and admits a particular decomposition under any $\mathbb{P} \in \mathcal{P}$.

Proposition 4.1. Under Assumptions 2.1, 2.2, for any $\mathbb{P} \in \mathcal{P}$, denoting by $\mathbb{G}_{+}^{\mathbb{P}}$ the usual augmentation of the right-limit of $\mathbb{G}$ under $\mathbb{P}$, there is a $\mathbb{G}_{+}^{\mathbb{P}}$-predictable process $\widetilde{Z}^{\mathbb{P}}$, which is also $\overline{\mathcal{F}}_{t}^{\mathbb{P}}-$ mesurable for a.e. $t \in[0, T]$, and a non-decreasing càdlàg and $\mathbb{G}_{+}^{\mathbb{P}}$-predictable process $\widetilde{K}^{\mathbb{P}}$, which is also $\overline{\mathcal{F}}_{t}^{\mathbb{P}}$-mesurable for a.e. $t \in[0, T]$, such that $V^{+}$defined by (4.5) satisfies for all $0 \leq t \leq s \leq T$

$$
V_{s}^{+}=\xi+\int_{s}^{T} \widehat{F}_{r}\left(V_{r}^{+}, \widetilde{Z}_{r}^{\mathbb{P}}\right) d s+\int_{s}^{T} g_{r}\left(V_{r}^{+}, \widetilde{Z}_{r}^{\mathbb{P}}\right) \cdot d \overleftarrow{W}_{r}-\int_{s}^{T} \widetilde{Z}_{r}^{\mathbb{P}} \cdot d B_{r}+\widetilde{K}_{T}^{\mathbb{P}}-\widetilde{K}_{s}^{\mathbb{P}}, \mathbb{P}-\text { a.s. }
$$

Proof. We introduce first the following RBDSDE with lower obstacle $V^{+}$under each $\mathbb{P} \in \mathcal{P}^{t}$,

$$
\left\{\begin{array}{l}
\widetilde{Y}_{t}^{\mathbb{P}}=\xi+\int_{t}^{T} \widehat{F}_{s}\left(\widetilde{Y}_{s}^{\mathbb{P}}, \widetilde{Z}_{s}^{\mathbb{P}}\right) d s+\int_{t}^{T} g_{s}\left(\widetilde{Y}_{s}^{\mathbb{P}}, \widetilde{Z}_{s}^{\mathbb{P}}\right) \cdot d \overleftarrow{W}_{s}-\int_{t}^{T} \widetilde{Z}_{s}^{\mathbb{P}} \cdot d B_{s}+\widetilde{K}_{T}^{\mathbb{P}}-\widetilde{K}_{t}^{\mathbb{P}} \\
\widetilde{Y}_{t}^{\mathbb{P}} \geq V_{t}^{+}, 0 \leq t \leq T, \mathbb{P}-a . s \\
\int_{0}^{T}\left(\widetilde{Y}_{s^{-}}^{\mathbb{P}}-V_{s^{-}}^{+}\right) d \widetilde{K}_{s^{-}}^{\mathbb{P}}=0, \mathbb{P}-\text { a.s. }
\end{array}\right.
$$

To the best of our knowledge, there are no results in the literature for the existence and uniqueness of such RBDSDE with càdlàg obstacle. The proofs of these results are postponed to Section $\mathrm{C}$ in the Appendix for completeness. As mentioned in Remark 4.9 in [64], and for a fixed $\mathbb{P} \in \mathcal{P}^{t}$, we shall use the solution of the above 
RBDSDEs and the notion of $\widehat{F}$-weak doubly super-martingale whis is introduced in the Appendix to prove the desired result. This notion is a natural extension of nonlinear $f$-super-martingale introduced first by Peng [54] in the context of standard BSDEs. Let us now argue by contradiction and suppose that $\widetilde{Y}^{\mathbb{P}}$ is not equal $\mathbb{P}-$ a.s. to $V^{+}$. Then we can assume without loss of generality that $\tilde{Y}_{0}^{\mathbb{P}}>V_{0}^{+}, \mathbb{P}-$ a.s. For each $\varepsilon>0$, define the following $\mathbb{G}$-stopping time

$$
\tau^{\varepsilon}:=\inf \left\{t \geq 0, \tilde{Y}_{t}^{\mathbb{P}} \leq V_{t}^{+}+\varepsilon\right\} .
$$

Then $\widetilde{Y}^{\mathbb{P}}$ is strictly above the obstacle before $\tau^{\varepsilon}$, and therefore $\widetilde{K}^{\mathbb{P}}$ is identically equal to 0 in $\left[0, \tau^{\varepsilon}\right]$. Hence, we have for all $0 \leq t \leq s \leq T$

$$
\widetilde{Y}_{s}^{\mathbb{P}}=\widetilde{Y}_{\tau^{\varepsilon}}^{\mathbb{P}}+\int_{s}^{\tau^{\varepsilon}} \widehat{F}_{r}\left(\widetilde{Y}_{r}^{\mathbb{P}}, \widetilde{Z}_{r}^{\mathbb{P}}\right) d r+\int_{s}^{\tau^{\varepsilon}} g_{r}\left(\widetilde{Y}_{r}^{\mathbb{P}}, \widetilde{Z}_{r}^{\mathbb{P}}\right) \cdot d \overleftarrow{W}_{r}-\int_{s}^{\tau^{\varepsilon}} \widetilde{Z}_{r}^{\mathbb{P}} \cdot d B_{r}, \mathbb{P}-a . s .
$$

Let us now define the following BDSDE on $\left[0, \tau^{\varepsilon}\right]$

$$
y_{s}^{+, \mathbb{P}}=V_{\tau^{\varepsilon}}^{+}+\int_{s}^{\tau^{\varepsilon}} \widehat{F}_{r}\left(y_{r}^{+, \mathbb{P}}, z_{r}^{+, \mathbb{P}}\right) d r+\int_{s}^{\tau^{\varepsilon}} g_{r}\left(y_{r}^{+, \mathbb{P}}, z_{r}^{+, \mathbb{P}}\right) \cdot d \overleftarrow{W}_{r}-\int_{s}^{\tau^{\varepsilon}} z_{r}^{+, \mathbb{P}} \cdot d B_{r}, \mathbb{P}-\text { a.s. }
$$

By comparison theorem and the standard a priori estimates, we obtain that

$$
\mathbb{E}\left[\widetilde{Y}_{0}^{\mathbb{P}}\right] \leq \mathbb{E}\left[y_{0}^{+, \mathbb{P}}\right]+C \mathbb{E}\left[\left|V_{\tau^{\varepsilon}}^{+}-\tilde{Y}_{\tau^{\varepsilon}}^{\mathbb{P}}\right|\right] \leq \mathbb{E}\left[y_{0}^{+, \mathbb{P}}\right]+C \varepsilon,
$$

by definition of $\tau^{\varepsilon}$.

Moreover, we can show similarly to the proof of Lemma 4.4 (see also the arguments in Step 1 of the proof of Theorem 4.5 in [64] pages 328-329) that $V^{+}$is a strong $\widehat{F}$-doubly super-martingale under each $\mathbb{P} \in \mathcal{P}^{t}$. Thus, we obtain in particular that $y_{0}^{+, \mathbb{P}} \leq V_{0}^{+}$which in turn implies

$$
\mathbb{E}\left[\tilde{Y}_{0}^{\mathbb{P}}\right] \leq \mathbb{E}\left[V_{0}^{+}\right]+C \varepsilon
$$

hence a contradiction by arbitrariness of $\varepsilon$.

We next prove a representation for $V^{+}$similar to (3.1), which will be useful for us to justify that the value process we have constructed provides indeed a solution to the 2BDSDE (2.1).

Proposition 4.2. Assume that Assumptions 2.1, 2.2 hold. Then we have

$$
V_{t}^{+}=\underset{\mathbb{P}^{\prime} \in \mathcal{P}(t+, \mathbb{P})}{\operatorname{ess} \sup ^{\mathbb{P}}} \mathcal{Y}_{t}^{\mathbb{P}^{\prime}}(T, \xi), \mathbb{P}-\text { a.s., } \forall \mathbb{P} \in \mathcal{P}^{t}
$$

Proof. The proof for the representations is the same as the proof of Lemma 3.5 in [58], since a stability result holds in our context, too.

\subsection{Existence result in the general case}

We are now in position to state the main result of this section.

Theorem 4.2. Let $\xi \in \mathcal{L}^{2}$ and assume that Assumptions 2.1, 2.2 hold. Then there exists a unique solution $(Y, Z, K) \in \mathbb{D}^{2} \times \mathbb{H}^{2} \times \mathbb{I}^{2}$ of the $2 B D S D E(2.1)$.

Proof. The proof is divided in three steps. In the first one we prove that the value process $V^{+}$defined by (4.5) is the solution of our 2BDSDE in the case when $\xi$ belongs in $\mathrm{UC}_{b}(\Omega)$ and show the aggregation result for 
the solution. Then, in the second step we verify the minimality condition for the increasing process. Finally, we deal with the general case.

Step 1: Existence and aggregation results for $\xi$ belongs in $\mathrm{UC}_{b}(\Omega)$. As we have mentioned above, the natural candidate for the $Y$ solution for our 2BDSDE is given by

$$
Y_{t}=V_{t}^{+}:=\lim _{r \in \mathbb{Q} \cap(t, T], r \downarrow t} V_{r},
$$

where $V$ is the value process defined by (4.2). First, we know that $V^{+}$is a càdlàg process defined path-wise and using the same notations in Proposition 4.1 our solution $Y$ verifies

$$
V_{t}^{+}=V_{0}^{+}-\int_{0}^{t} \widehat{F}_{s}\left(V_{s}^{+}, \widetilde{Z}_{s}^{\mathbb{P}}\right) d s-\int_{0}^{t} g_{s}\left(V_{s}^{+}, \widetilde{Z}_{s}^{\mathbb{P}}\right) \cdot d \overleftarrow{W}_{s}+\int_{0}^{t} \widetilde{Z}_{s}^{\mathbb{P}} \cdot d B_{s}-\widetilde{K}_{t}^{\mathbb{P}}, \mathbb{P}-\text { a.s., } \quad \forall \mathbb{P} \in \mathcal{P}^{t}
$$

We note that $V^{+}$is $(\mathbb{P}$ - a.s.) a càdlàg generalized semi-martingale under any $\mathbb{P} \in \mathcal{P}$, (studied by Pardoux and Protter in [53] and Pardoux and Peng [52]). By the generalized Itô's formula of Lemma A.1, we have for any $i=1, \ldots, d$

$$
\begin{aligned}
B_{t}^{i} V_{t}^{+}= & \int_{0}^{t}\left(\widehat{a}_{s}^{1 / 2} \widetilde{Z}_{s}^{\mathbb{P}} \cdot \mathbf{1}_{i}-\widehat{F}_{s}\left(V_{s}^{+}, \widetilde{Z}_{s}^{\mathbb{P}}\right) B_{s}^{i}\right) d s+\int_{0}^{t}\left(\widetilde{Z}_{s}^{\mathbb{P}} B_{s}^{i}+\mathbf{1}_{d} V_{s}^{+}\right) \cdot d B_{s} \\
& -\int_{0}^{t} g_{s}\left(V_{s}^{+}, \widetilde{Z}_{s}^{\mathbb{P}}\right) B_{s}^{i} \cdot d \overleftarrow{W}_{s}-\int_{0}^{t} B_{s}^{i} d \widetilde{K}_{s}^{\mathbb{P}} \\
= & \int_{0}^{t} \widehat{a}_{s}^{1 / 2} \widetilde{Z}_{s}^{\mathbb{P}} \cdot \mathbf{1}_{i} d s+\int_{0}^{t} B_{s}^{i} d V_{s}^{+}+\int_{0}^{t} \mathbf{1}_{d} V_{s}^{+} \cdot d B_{s} .
\end{aligned}
$$

Then, we can adapt Karandikar's results obtained for càdlàg semi-martingale in our context to define universally the two stochastic integrals

$$
\int_{0}^{t} B_{s}^{i} d V_{s}^{+}, \text {and } \int_{0}^{t} \mathbf{1}_{d} V_{s}^{+} \cdot d B_{s} .
$$

Indeed, $B$ and $V^{+}$are both càdlàg and a backward Itô integral can always be considered as a forward Itô integral, provided that time is reversed.

This gives us the existence of a $\mathbb{G}$-predictable process $Z$ such that for any $\mathbb{P} \in \mathcal{P}$

$$
\widetilde{Z}_{t}^{\mathbb{P}}=Z_{t}, d t \otimes \mathbb{P}-\text { a.e. }
$$

Furthermore, since for any $\mathbb{P} \in \mathcal{P}, \widetilde{Z}_{t}$ is $\overline{\mathcal{F}}_{t}^{\mathbb{P}}$-mesurable for a.e. $t \in[0, T]$, we deduce immediately that $Z_{t}$ is $\bigcap_{\mathbb{P} \in \mathcal{P}} \overline{\mathbb{F}}_{t}^{\mathbb{P}}$-mesurable for a.e. $t \in[0, T]$.

Concerning the fact that we can aggregate the family $\left(\widetilde{K}^{\mathbb{P}}\right)_{\mathbb{P} \in \mathcal{P}}$, it can be deduced as follows. We have from (4.5) that $V^{+}$is defined path-wise, and so is the Lebesgue integral $\int_{0}^{t} \widehat{F}_{s}\left(V_{s}^{+}, Z_{s}\right) d s$. By [47], the stochastic integrals $\int_{0}^{t} Z_{s} \cdot d B_{s}$ and $\int_{0}^{t} g_{s}\left(V_{s}^{+}, Z_{s}\right) \cdot d \overleftarrow{W}_{s}$ can also be defined path-wise. We can therefore define path-wise

$$
K_{t}:=V_{0}^{+}-V_{t}^{+}-\int_{0}^{t} \widehat{F}_{s}\left(V_{s}^{+}, Z_{s}\right) d s-\int_{0}^{t} g_{s}\left(V_{s}^{+}, Z_{s}\right) \cdot d \overleftarrow{W}_{s}+\int_{0}^{t} Z_{s} \cdot d B_{s}
$$

and $K$ is an aggregator for the family $\left(\widetilde{K}^{\mathbb{P}}\right)_{\mathbb{P} \in \mathcal{P}^{t}}$. Thus, the triplet $(Y, Z, K)$ satisfies the equation $(2.1)$ and from the a priori estimates in Theorem 3.1 we get that $(Y, Z, K)$ belongs to $\mathbb{D}^{2} \times \mathbb{H}^{2} \times \mathbb{I}^{2}$.

Step 2: The minimality condition of $\widetilde{K}$. Now, we have to check that the minimum condition (2.3) holds. We follow the arguments in the proof of Theorem 3.1. For $t \in[0, T], \mathbb{P} \in \mathcal{P}^{t}$ and $\mathbb{P}^{\prime} \in \mathcal{P}(t+, \mathbb{P})$, we denote 
$\delta Y:=V^{+}-y^{\mathbb{P}^{\prime}}(T, \xi)$ and $\delta Z:=Z-z^{\mathbb{P}^{\prime}}(T, \xi)$ and we introduce the process $M$ of (3.2). We first observe that since $K$ is non-decreasing, we have

$$
\underset{\mathbb{P}^{\prime} \in \mathcal{P}\left(t^{+}, \mathbb{P}\right)}{\operatorname{essinf}} \mathbb{E}_{t}^{\mathbb{P}^{\prime}}\left[K_{T}-K_{t}\right] \geq 0
$$

Then, it suffices to prove that $\mathbb{E}^{\mathbb{P}}\left[\underset{\mathbb{P}^{\prime} \in \mathcal{P}\left(t^{+}, \mathbb{P}\right)}{\operatorname{essinf}} \mathbb{E}_{t}^{\mathbb{P}} \mathbb{P}^{\prime}\left[K_{T}-K_{t}\right]\right] \leq 0$. We know that the family $\mathcal{P}\left(t^{+}, \mathbb{P}\right)$ is upward directed. Therefore, by classical results, there is a sequence $\left(\mathbb{P}^{n}\right)_{n \geq 0} \subset \mathcal{P}\left(t^{+}, \mathbb{P}\right)$ such that

$$
\mathbb{E}^{\mathbb{P}}\left[\underset{\mathbb{P}^{\prime} \in \mathcal{P}\left(t^{+}, \mathbb{P}\right)}{\operatorname{essinf}} \mathbb{E}_{t}^{\mathbb{P}^{\prime}}\left[K_{T}-K_{t}\right]\right]=\lim _{n \rightarrow+\infty} \downarrow \mathbb{E}^{\mathbb{P}^{n}}\left[K_{T}-K_{t}\right] .
$$

On the other hand, by (3.3), we estimate by the Hölder inequality that

$$
\begin{aligned}
\mathbb{E}^{\mathbb{P}^{n}}\left[K_{T}-K_{t}\right] & =\mathbb{E}^{\mathbb{P}^{n}}\left[\left(\inf _{t \leq s \leq T}\left(M_{t}^{-1} M_{s}\right)\right)^{1 / 3}\left(K_{T}-K_{t}\right)^{1 / 3}\left(\inf _{t \leq s \leq T}\left(M_{t}^{-1} M_{s}\right)\right)^{-1 / 3}\left(K_{T}-K_{t}\right)^{2 / 3}\right] \\
& \leq C\left(\mathbb{E}^{\mathbb{P}^{n}}\left[\left(K_{T}^{\mathbb{P}^{n}}\right)^{2}\right] \mathbb{E}^{\mathbb{P}^{n}}\left[\left(\inf _{t \leq s \leq T}\left(M_{t}^{-1} M_{s}\right)\right)\left(K_{T}-K_{t}\right)\right]\right)^{1 / 3} \\
& \leq C\left(\mathbb{E}^{\mathbb{P}^{n}}\left[\left(K_{T}\right)^{2}\right] \mathbb{E}^{\mathbb{P}^{n}}\left[M_{t}^{-1} \int_{t}^{T} M_{s} d K_{s}\right]\right)^{1 / 3} \\
& \leq C\left(\mathbb{E}^{\mathbb{P}^{n}}\left[\left(K_{T}\right)^{2}\right]\right)^{1 / 3}\left(\mathbb{E}^{\mathbb{P}^{n}}\left[\delta Y_{t}\right]\right)^{1 / 3},
\end{aligned}
$$

where we have used in the last inequality the fact that $K$ is non-decreasing and the same arguments as in the proof of Theorem 3.1 (ii).

Plugging the above in (4.7), we obtain

$$
\mathbb{E}^{\mathbb{P}}\left[\underset{\mathbb{P}^{\prime} \in \mathcal{P}\left(t^{+}, \mathbb{P}\right)}{\operatorname{essinf}} \mathbb{P}_{t}^{\mathbb{P}} \mathbb{E}_{t}^{\mathbb{P}^{\prime}}\left[K_{T}-K_{t}\right]\right] \leq C \lim _{n \rightarrow+\infty} \downarrow\left(\mathbb{E}^{\mathbb{P}^{n}}\left[\delta Y_{t}\right]\right)^{1 / 3} \leq C\left(\underset{\mathbb{P}^{n} \in \mathcal{P}\left(t^{+}, \mathbb{P}\right)}{\operatorname{essinf}} \mathbb{E}^{\mathbb{P}} \mathbb{P}^{n}\left[\delta Y_{t}\right]\right)^{1 / 3}=0 .
$$

which is the desired result.

Step 3: Existence and aggregation results for $\xi$ belonging to $\mathcal{L}^{2}$. For $\xi \in \mathcal{L}^{2}$, there exists by definition a sequence $\left(\xi_{n}\right)_{n \geq 0} \subset U C_{b}(\Omega)$ such that

$$
\lim _{n \rightarrow+\infty}\left\|\xi_{n}-\xi_{m}\right\|_{\mathbb{L}^{2}}=0 \quad \text { and } \quad \sup _{n \geq 0}\left\|\xi_{n}\right\|_{\mathbb{L}^{2}}<+\infty .
$$

Let $\left(Y^{n}, Z^{n}, K^{n}\right) \in \mathbb{D}^{2} \times \mathbb{H}^{2} \times \mathbb{I}^{2}$ be the solution to $2 \operatorname{BDSDE}(2.1)$ with terminal condition $\xi_{n}$. By the estimates of Theorem 3.4, we have

$$
\begin{aligned}
& \left\|Y^{n}-Y^{m}\right\|_{\mathbb{D}^{2}}^{2}+\left\|Z^{n}-Z^{m}\right\|_{\mathbb{H}^{2}}^{2}+\sup _{\mathbb{P} \in \mathcal{P}} \mathbb{E}^{\mathbb{P}}\left[\sup _{0 \leq t \leq T}\left|\widetilde{K}_{t}^{n}-\widetilde{K}_{t}^{m}\right|^{2}\right] \\
& \leq C\left\|\xi_{n}-\xi_{m}\right\|_{\mathbb{L}^{2}}^{2}+\widetilde{C}\left\|\xi_{n}-\xi_{m}\right\|_{\mathbb{L}^{2}} \underset{n, m \rightarrow+\infty}{\longrightarrow} 0 .
\end{aligned}
$$

Extracting a fast-converging subsequence and using Borel-Cantelli Lemma, we can make sure that, $\mathbb{P}-$ a.s.

$$
\lim _{n \rightarrow \infty}\left[\sup _{0 \leq t \leq T}\left[\left|Y_{t}^{n}-Y_{t}^{m}\right|^{2}+\left|\widetilde{K}_{t}^{n}-\widetilde{K}_{t}^{m}\right|^{2}\right]+\int_{0}^{T}\left\|\widehat{a}_{t}^{1 / 2}\left(Z_{t}^{n}-Z_{t}^{m}\right)\right\|^{2} d t\right]=0 .
$$

Define then

$$
Y:=\varlimsup_{n \rightarrow \infty} Y^{n}, \quad Z:=\varlimsup_{n \rightarrow \infty} Z^{n}, \quad K:=\varlimsup_{n \rightarrow \infty} \widetilde{K}^{n},
$$

It is therefore clear that $(Y, Z, K) \in \mathbb{D}^{2} \times \mathbb{H}^{2} \times \mathbb{I}^{2}$. 


\section{Probabilistic interpretation for fully-nonlinear SPDEs}

The aim of this section is to give a Feynman-Kac's formula for the solution of the following fully non-linear SPDEs

$$
\left\{\begin{array}{l}
d u(t, x)+\hat{h}\left(t, x, u(t, x), D u(t, x), D^{2} u(t, x)\right) d t+g(t, x, u(t, x), D u(t, x)) \circ d \overleftarrow{W}_{t}=0 \\
u(T, x)=\phi(x)
\end{array}\right.
$$

where we consider the case $H_{t}(\omega, y, z, \gamma)=h\left(t, B_{t}(\omega), y, z, \gamma\right)$, with $h:[0, T] \times \mathbb{R} \times \mathbb{R}^{d} \times D_{h} \longrightarrow \mathbb{R}$ (with $D_{h}$ being a subset of $\mathbb{S}_{d}^{>0}$ ) is a deterministic map. Then, the corresponding conjuguate and bi-conjuguate functions are given by

$$
\begin{aligned}
& F(t, x, y, z, a):=\sup _{\gamma \in D_{h}}\left\{\frac{1}{2} \operatorname{Tr}[a \gamma]-h(t, x, y, z, \gamma)\right\} \text { for } a \in \mathbb{S}_{d}^{>0} \\
& \hat{h}(t, x, y, z, \gamma):=\sup _{a \in \mathbb{S}_{d}^{>0}}\left\{\frac{1}{2} \operatorname{Tr}[a \gamma]-F(t, x, y, z, a)\right\} \text { for } \gamma \in \mathbb{R}^{d \times d} .
\end{aligned}
$$

Notice that $-\infty<\hat{h} \leq h$ and $\hat{h}$ is nondecreasing convex in $\gamma$. Also, $\hat{h}=h$ if and only if $h$ is convex and nondecreasing in $\gamma$, which we will therefore always assume.

For this end, the following markovian 2BDSDE is considered

$$
\begin{aligned}
Y_{s}^{t, x}= & \phi\left(B_{T}^{t, x}\right)-\int_{s}^{T} F\left(s, B_{r}^{t, x}, Y_{r}^{t, x}, Z_{r}^{t, x}, \widehat{a}_{r}\right) d r+\int_{t}^{T} g\left(r, B_{r}^{t, x}, Y_{r}^{t, x}, Z_{r}^{t, x}\right) \circ d \overleftarrow{W}_{r} \\
& -\int_{s}^{T} Z_{r}^{t, x} d B_{r}^{t, x}+K_{T}^{t, x}-K_{s}^{t, x}, t \leq s \leq T, \mathcal{P}^{t}-q . s,
\end{aligned}
$$

where for any $(t, x) \in[0, T] \times \mathbb{R}^{d},\left(B_{s}^{t}\right)_{s \in[t, T]}$ is the shifted canonical process on $\Omega^{B, t}$ defined by

$$
B_{s}^{t, x}:=x+B_{s}^{t} \quad \text { for all } s \in[t, T] .
$$

The stochastic integral with respect to $\overleftarrow{W}$ is the Stratonovich backward integral (see Kunita [40] page 194). Using the definition of the Stratonovich backward integral, we can show easily that equation (5.4) is equivalent to the following $2 \mathrm{BDSDE}$

$$
\begin{aligned}
Y_{s}^{t, x}= & \phi\left(B_{T}^{t, x}\right)-\int_{s}^{T} f\left(s, B_{r}^{t, x}, Y_{r}^{t, x}, Z_{r}^{t, x}, \widehat{a}_{r}\right) d r+\int_{t}^{T} g\left(r, B_{r}^{t, x}, Y_{r}^{t, x}, Z_{r}^{t, x}\right) \cdot d \overleftarrow{W}_{r} \\
& -\int_{s}^{T} Z_{r}^{t, x} d B_{r}^{t, x}+K_{T}^{t, x}-K_{s}^{t, x}, t \leq s \leq T, \mathcal{P}^{t}-q . s
\end{aligned}
$$

where

$$
f\left(s, x, y, z, \widehat{a}_{s}\right):=F\left(s, x, y, z, \widehat{a}_{s}\right)+\frac{1}{2} \operatorname{Tr}\left[g(s, x, y, z) D_{y} g(s, x, y, z)^{\top}\right] .
$$

From now on, we focus our study on providing the probabilistic representation of the classical and stochastic viscosity solutions for the fully nonlinear SPDEs (5.1) via 2BDSDEs (5.4). Let us first define the following functional spaces:

- $\mathcal{M}_{0, T}^{W}$ denotes all the $\mathbb{F}^{W}$-stopping times $\tau$ such that $0 \leq \tau \leq T$.

- $L^{p}\left(\mathcal{F}_{\tau, T}^{W} ; \mathbb{R}^{d}\right)$, for $p \geq 0$, denotes the space of all $\mathbb{R}^{d}$-valued $\mathcal{F}_{\tau, T}^{W}-$ measurable r.v. $\xi$ such that $\mathbb{E}\left[|\xi|^{p}\right]<$ $+\infty$. 
- $C^{\ell, k}\left([0, T] \times \mathbb{R}^{d}\right)$, for $k, \ell \geq 0$, denotes the space of all $\mathbb{R}$-valued functions defined on $[0, T] \times \mathbb{R}^{d}$, which are $\ell$-times continuously differentiable in $t, k$-times continuously differentiable in $x$.

- $C_{b}^{k, m, n}\left([0, T] \times \mathbb{R}^{d} \times \mathbb{R} ; \mathbb{R}^{p}\right)$, for $k, m, n \geq 0, p \geq 1$, denotes the space of all $\mathbb{R}^{p}$ - valued functions defined on $[0, T] \times \mathbb{R}^{d} \times \mathbb{R}$, which are $k$-times continuously differentiable in $t, m$-times continuously differentiable in $x, n$-times continuously differentiable in $y$ and have uniformly bounded partial derivatives.

- $C^{\ell, k}\left(\mathcal{F}_{t, T}^{W},[0, T] \times \mathbb{R}^{d}\right)$, for $k, \ell \geq 0$, denotes the space of all $C^{\ell, k}\left([0, T] \times \mathbb{R}^{d}\right)$-valued random variables $\varphi$ that are $\mathcal{F}_{t, T}^{W} \otimes \mathcal{B}\left([0, T] \times \mathbb{R}^{d}\right)$-measurable.

- $C^{\ell, k}\left(\mathbb{F}^{W},[0, T] \times \mathbb{R}^{d}\right)$, for $k, \ell \geq 0$, denotes the space of r.v. $\varphi \in C^{\ell, k}\left(\mathcal{F}_{t, T}^{W},[0, T] \times \mathbb{R}^{d}\right)$ such that for fixed $x \in \mathbb{R}^{d}$, the mapping $(t, \omega) \longmapsto \varphi(t, x, \omega)$ is $\mathbb{F}^{W}$-progressively measurable.

Furthermore, for $(t, x, y) \in[0, T] \times \mathbb{R}^{d} \times \mathbb{R}$, we denote $\partial / \partial y=D_{y}, \partial / \partial t=D_{t}, D=D_{x}=\left(\partial / \partial x_{1}, \cdots, \partial / \partial x_{d}\right)$, and $D^{2}=D_{x x}=\left(\partial_{x_{i} x_{j}}^{2}\right)_{i, j=1}^{d}$. The meaning of $D_{x y}, D_{y y}, \ldots$, should be clear.

Then, we list the assumptions needed in this section. The following is a slight strengthening of Assumption 2.1, where we assume a bit more regularity.

\section{Assumption 5.1.}

(i) $\mathcal{P}$ is not empty, the domain $D_{F_{t}(y, z)}=D_{F_{t}}$ is independent of $(w, y, z)$. Moreover, $F, g$ and $D_{y} g$ are uniformly continuous in $t$, uniformly in a on $D_{F_{t}}$.

(ii) There exist constants $C>0,0 \leq \alpha<1$ such that for all $\left(t, a, x, x^{\prime}, z, z^{\prime}, y, y^{\prime}\right) t \in[0, T] \times D_{F_{t}} \times\left(\mathbb{R}^{d}\right)^{4} \times \mathbb{R}^{2}$

$$
\begin{aligned}
\left|F(t, x, y, z, a)-F\left(t, x^{\prime}, y^{\prime}, z^{\prime}, a\right)\right| & \leq C\left(\left|x-x^{\prime}\right|+\left|y-y^{\prime}\right|+\left\|a^{1 / 2}\left(z-z^{\prime}\right)\right\|\right), \\
\left\|g(t, x, y, z)-g\left(t, x^{\prime}, y^{\prime}, z^{\prime}\right)\right\|^{2} & \leq C\left(\left|x-x^{\prime}\right|^{2}+\left|y-y^{\prime}\right|^{2}\right)+\alpha\left\|\left(z-z^{\prime}\right)\right\|^{2} .
\end{aligned}
$$

(iii) The function $g$ belongs to $C_{b}^{0,2,3}\left([0, T] \times \mathbb{R}^{d} \times \mathbb{R} ; \mathbb{R}^{l}\right)$.

(iv) There exists a constant $\lambda \in[0,1[$ such that

$$
(1-\lambda) \widehat{a}_{t} \geq \alpha I_{d}, d t \times \mathcal{P}-q . e .
$$

We next state a strengthened version of Assumption 2.2 in the present Markov framework.

Assumption 5.2. (i) The function $\phi$ is a uniformly continuous and bounded function on $\mathbb{R}^{d}$.

(ii) For any $(t, x) \in[0, T] \times \mathbb{R}^{d}$ and for some $\varepsilon>0$

$$
\sup _{\mathbb{P} \in \mathcal{P}^{t}} \mathbb{E}^{\mathbb{P}}\left[\left|\phi\left(B_{T}^{t, x}\right)\right|^{2+\varepsilon}+\int_{t}^{T}\left|F\left(s, B_{s}^{t, x}, 0,0, \widehat{a}_{s}^{t}\right)\right|^{2+\varepsilon} d s+\int_{t}^{T}\left\|g\left(s, B_{s}^{t, x}, 0,0\right)\right\|^{2+\varepsilon} d s\right]<+\infty .
$$

Therefore under Assumptions 2.3, 5.1 and 5.2 and according to Theorem 4.2, there exists a unique triplet $\left(Y^{t, x}, Z^{t, x}, K^{t, x}\right)$ solution of the 2BDSDE (5.4). Indeed, it is immediate to check that $f$ and $g$ indeed satisfy Assumption 2.1 (recall that $D_{y} g$ is bounded) and that the terminal condition also verifies all the required regularity and integrability properties.

\subsection{Classical solution of SPDEs}

We can rewrite the $\operatorname{SPDE}(5.1)$ in its so-called integral form, as soon as $\left\{u(t, x), 0 \leq t \leq T, x \in \mathbb{R}^{d}\right\} \in$ $C^{0,2}\left(\mathcal{F}_{t, T}^{W},[0, T] \times \mathbb{R}^{d}\right)$ is a classical solution of the following equation where the stochastic integral is written in the Stratonovich form, namely,

$$
u(t, x)=\phi(x)+\int_{t}^{T} \hat{h}\left(t, x, u(t, x), D u(t, x), D^{2} u(t, x)\right) d t+\int_{t}^{T} g(t, x, u(t, x), D u(t, x)) \circ d \overleftarrow{W}_{t} .
$$


Definition 5.1. We define a classical solution of the SPDE (5.1) as a $\mathbb{R}$-valued random field $\{u(t, x),(t, x) \in$ $\left.[0, T] \times \mathbb{R}^{d}\right\}$ such that $u(t, x)$ is $\mathcal{F}_{t, T}^{W}-$ measurable for each $(t, x)$, and whose trajectories belong to $C^{0,2}\left([0, T] \times \mathbb{R}^{d}\right)$.

The following is a version of the celebrated Feynman-Kac formula in the present context.

Theorem 5.1. Let Assumption 5.1 hold true. Suppose further that $H$ is continuous in its domain, $D_{F}$ is independent of $t$ and is bounded both from above and away from 0 . Let $\left\{u(t, x),(t, x) \in[0, T] \times \mathbb{R}^{d}\right\}$ be a classical solution of (5.1) with $\left\{(u, D u)\left(s, B_{s}^{t, x}\right), s \in[t, T]\right\} \in \mathbb{D}^{2} \times \mathbb{H}^{2}$. Then

$$
Y_{s}^{t, x}:=u\left(s, B_{s}^{t, x}\right), Z_{s}^{t, x}:=D u\left(s, B_{s}^{t, x}\right), K_{s}^{t, x}:=\int_{0}^{s} k_{r} d r,
$$

with

$$
k_{s}:=\hat{h}\left(s, B_{s}, Y_{s}, Z_{s}, \Gamma_{s}\right)-\frac{1}{2} \operatorname{Tr}\left[\widehat{a}_{s} \Gamma_{s}\right]+F\left(s, B_{s}, Y_{s}, Z_{s}, \widehat{a}_{s}\right) \text { and } \Gamma_{s}:=D^{2} u\left(s, B_{s}^{t, x}\right),
$$

is the unique solution of the $2 B D S D E$ (5.4). Moreover, $u(t, x)=Y_{t}^{t, x}$ for all $t \in[0, T]$.

Proof. It suffices to show that $(Y, Z, K)$ solves the 2BDSDE (5.4). Let $s=t_{0}<t_{1}<t_{2}<\ldots<t_{n}=T$, then writing $B$ instead of $B^{t, x}$ and $B_{i}$ instead of $B_{t_{i}}$ for notational simplicity, we have

$$
\begin{aligned}
& \sum_{i=0}^{n-1}\left[u\left(t_{i}, B_{i}\right)-u\left(t_{i+1}, B_{i+1}\right)\right]=\sum_{i=0}^{n-1}\left[u\left(t_{i}, B_{i}\right)-u\left(t_{i}, B_{i+1}\right)\right]+\sum_{i=0}^{n-1}\left[u\left(t_{i}, B_{i+1}\right)-u\left(t_{i+1}, B_{i+1}\right)\right] \\
& =-\sum_{i=0}^{n-1} \int_{t_{i}}^{t_{i+1}} D u\left(t_{i}, B_{r}\right) d B_{r}-\sum_{i=0}^{n-1} \int_{t_{i}}^{t_{i+1}} \frac{1}{2} \operatorname{Tr}\left[\widehat{a}_{r} D^{2} u\left(t_{i}, B_{r}\right)\right] d r \\
& \quad+\sum_{i=0}^{n-1} \int_{t_{i}}^{t_{i+1}} \hat{h}\left(r, B_{i+1}, u\left(r, B_{i+1}^{t, x}\right), D u\left(r, B_{i+1}\right), D^{2} u\left(r, B_{i+1}\right)\right) d r \\
& \quad+\sum_{i=0}^{n-1} \int_{t_{i}}^{t_{i+1}} g\left(r, B_{i+1}, u\left(r, B_{i+1}\right), D u\left(r, B_{i+1}\right)\right) \circ d \overleftarrow{W}_{s},
\end{aligned}
$$

where we have used the Itô formula and the Equation (5.6) satisfied by $u$. Now, the transformation from Stratonovich to Itô integral yields

$$
\begin{aligned}
& \sum_{i=0}^{n-1}\left[u\left(t_{i}, B_{i}\right)-u\left(t_{i+1}, B_{i+1}\right)\right]=-\sum_{i=0}^{n-1} \int_{t_{i}}^{t_{i+1}} D u\left(t_{i}, B_{r}\right) d B_{r}-\sum_{i=0}^{n-1} \int_{t_{i}}^{t_{i+1}} \frac{1}{2} \operatorname{Tr}\left[\widehat{a}_{r} D^{2} u\left(t_{i}, B_{r}\right)\right] d r \\
& \quad+\sum_{i=0}^{n-1} \int_{t_{i}}^{t_{i+1}} \hat{h}\left(r, B_{i+1}, u\left(r, B_{i+1}\right), D u\left(r, B_{i+1}\right), D^{2} u\left(r, B_{i+1}\right)\right) d r \\
& \left.\quad+\sum_{i=0}^{n-1} \int_{t_{i}}^{i+1} g\left(r, B_{i+1}\right), u\left(r, B_{i+1}\right), D u\left(r, B_{i+1}\right)\right) d \overleftarrow{W}_{r}+\sum_{i=0}^{n-1} \int_{t_{i}}^{t_{i+1}} F\left(r, B_{i+1}, u\left(r, B_{i+1}\right), D u\left(r, B_{i+1}\right), \widehat{a}_{r}\right) d r \\
& \quad-\sum_{i=0}^{n-1} \int_{t_{i}}^{t_{i+1}} F\left(r, B_{i+1}, u\left(r, B_{i+1}\right), D u\left(r, B_{i+1}\right), \widehat{a}_{r}\right) d r \\
& \quad-\frac{1}{2} \sum_{i=0}^{n-1} \int_{t_{i}}^{t_{i+1}} \operatorname{Tr}\left[g\left(r, B_{i+1}, u\left(r, B_{i+1}\right), D u\left(r, B_{i+1}\right)\right) D g\left(r, B_{i+1}, u\left(r, B_{i+1}\right)\right), D u\left(r, B_{i+1}\right)\right] d r .
\end{aligned}
$$

It then suffices to let the mesh size go to zero to obtain that the processes $(Y, Z, K)$ we have defined do satisfy Equation (5.4). It now remains to prove the minimum condition

$$
\underset{\mathbb{P}^{\prime} \in \mathcal{P}(t+, \mathbb{P})}{\operatorname{essinf}} \underset{\mathbb{P}}{\mathbb{P}_{t}^{\mathbb{P}^{\prime}}}\left[\int_{t}^{T} k_{s} d s\right]=0 \text { for all } t \in[0, T], \mathbb{P} \in \mathcal{P}
$$


by which we can conclude that $(Y, Z, K)$ is a solution of the 2BDSDE (5.4), provided that (5.7) holds. However, the proof of that (technical) point can actually be carried out exactly as in [65, Theorem 5.3] or [37, Theorem 5.3]. Indeed, the main point is that one has to be able to construct appropriate strong solutions to some SDEs on $\Omega^{B}$, similar to the ones in Example 4.5 of [63]. In our framework, this construction can be carried about for every fixed $\omega^{W}$, and it then suffices to use once more the results of Stricker and Yor [66].

\subsection{Stochastic viscosity solution for SPDE}

The aim of this section is to give a probabilistic representation for the stochastic viscosity solutions of the following fully non-linear SPDEs via solutions of 2BDSDEs (5.4). We restrict our study to the following class of SPDEs where the coefficient $g$ does not depends on the gradient of the solution,

$$
\left\{\begin{array}{l}
d u(t, x)+\hat{h}\left(t, x, u(t, x), D u(t, x), D^{2} u(t, x)\right) d t+g(t, x, u(t, x)) \circ d \overleftarrow{W}_{t}=0, \\
u(T, x)=\phi(x),
\end{array}\right.
$$

As mentioned in the introduction, Lions and Souganidis have introduced a notion of stochastic viscosity solution for fully nonlinear SPDEs in $[42,43,44]$ motivated by applications in path-wise stochastic control problems and the associated stochastic HJB equations. Buckdahn and Ma $[9,10]$ have introduced the rigorous notion of stochastic viscosity solution for semi-linear SPDEs and have then given the probabilistic interpretation of such equation via BDSDEs, where the intensity of the noise $g$ in the SPDEs (5.8) does not depend on the gradient of the solution. As mentioned in the introduction, they used the so-called Doss-Sussmann transformation and stochastic diffeomorphism flow technics to convert the semi-linear SPDEs to PDEs with random coefficients. This transformation permits to remove the stochastic integral term from the SPDEs and then gives a rigorous definition of so-called stochastic viscosity solution for SPDEs. We have to mention that it is difficult to define viscosity solution for SPDEs due to the fact that there are no maximum principle for solutions of SPDEs, because of the presence of the stochastic integral term in the equation. Since we are following a similar approach, this explains why we also assume that the non-linearity $g$ is not impacted by the gradient term.

We will use the shifted probability spaces defined in Section 4. We now introduce the random function $u$ : $[0, T] \times \Omega^{W} \times \mathbb{R}^{d} \longrightarrow \mathbb{R}$ given by

$$
u(t, x):=Y_{t}^{t, x}=\sup _{\mathbb{P} \in \mathcal{P}^{t}} y_{t}^{\mathbb{P}, t, x}, \text { for }(t, x) \in[0, T] \times \mathbb{R}^{d},
$$

where for any $(t, x, \mathbb{P}) \in[0, T] \times \mathbb{R}^{d} \times \mathcal{P}^{t},\left(y^{\mathbb{P}, t, x}, z^{\mathbb{P}, t, x}\right)$ is the unique solution of the BDSDE

$$
\begin{aligned}
y_{s}^{\mathbb{P}, t, x}= & \phi\left(B_{T}^{t, x}\right)-\int_{s}^{T} f\left(r, B_{r}^{t, x}, y_{r}^{\mathbb{P}, t, x}, z_{r}^{\mathbb{P}, t, x}, \widehat{a}_{r}\right) d r+\int_{t}^{T} g\left(r, B_{r}^{t, x}, y_{r}^{\mathbb{P}, t, x}, z_{r}^{\mathbb{P}, t, x}\right) \cdot d \overleftarrow{W}_{r} \\
& -\int_{s}^{T} z_{r}^{\mathbb{P}, t, x} \cdot d B_{r}^{t, x}, t \leq s \leq T, \mathbb{P}-a . s .
\end{aligned}
$$

By the Blumenthal $0-1$ law, it follows that $u(t, x)$ is deterministic with respect to $B$, but still an $\mathbb{F}^{W}$-adapted process.

Theorem 5.2. Let Assumptions 2.3, 5.1 and 5.2 hold true. Then $u$ belongs to $C\left(\mathcal{F}_{t, T}^{W},[0, T] \times \mathbb{R}^{d}\right)$.

Proof. Let us start with the uniform continuity in $x$. For any $(t, x),\left(t, x^{\prime}\right) \in[0, T] \times \mathbb{R}^{d}$, we have for any $\mathbb{P} \in \mathcal{P}^{t}$

$$
\mathbb{E}^{\mathbb{P}_{0}^{W}}\left[\left|u(t, x)-u\left(t, x^{\prime}\right)\right|^{2}\right]=\mathbb{E}^{\mathbb{P}_{0}^{W}}\left[\left|\sup _{\mathbb{P} \in \mathcal{P}^{t}} y_{t}^{\mathbb{P}, t, x}-\sup _{\mathbb{P} \in \mathcal{P}^{t}} y_{t}^{\mathbb{P}, t, x^{\prime}}\right|^{2}\right] \leq \sup _{\mathbb{P} \in \mathcal{P}^{t}} \mathbb{E}^{\mathbb{P}_{0}^{W}}\left[\left|y_{t}^{\mathbb{P}, t, x}-y_{t}^{\mathbb{P}, t, x^{\prime}}\right|^{2}\right] .
$$


But by the classical a priori estimates for BDSDEs, and using in particular the fact that $\phi$ is Lipschitz continuous, and $f$ and $g$ are Lipschitz continuous in $x$, we have for any $p \in[1,2]$

$$
\mathbb{E}^{\mathbb{P}_{0}^{W}}\left[\left|y_{t}^{\mathbb{P}, t, x}-y_{t}^{\mathbb{P}, t, x^{\prime}}\right|^{p}\right] \leq C \mathbb{E}^{\mathbb{P}_{0}^{W}}\left[\left|x-x^{\prime}\right|^{p}+\int_{t}^{T}\left|B_{s}^{t, x}-B_{s}^{t, x^{\prime}}\right|^{p} d s\right] \leq C\left\|x-x^{\prime}\right\|^{p} .
$$

By Kolmogorov-Chentsov's Theorem, this implies immediately that a $\mathbb{P}_{0}^{W}$-version of $u$ is $(1 / 2-\eta)$-Hölder continuous in $x$, for any $\eta \in(0,1 / 2)$.

Next, for any $\left(t, t^{\prime}, x\right) \in[0, T]^{2} \times \mathbb{R}^{d}$, we have the following classical dynamic programming result

$$
Y_{t^{\prime}}^{t, x}=u\left(t^{\prime}, B_{t^{\prime}}^{t, x}\right),
$$

which implies that for any $\mathbb{P} \in \mathcal{P}^{t}$ and any $\varepsilon^{\prime} \in(0, \varepsilon)$, using the estimates of Theorem 3.4(i)

$$
\begin{aligned}
& \mathbb{E}^{\mathbb{P}_{0}^{W}}\left[\left|u\left(t^{\prime}, x\right)-u(t, x)\right|^{2+\varepsilon^{\prime}}\right] \\
& =\mathbb{E}^{\mathbb{P}_{0}^{W}}\left[\left|u\left(t^{\prime}, x\right)-u\left(t^{\prime}, B_{t^{\prime}}^{t, x}\right)+Y_{t^{\prime}}^{t, x}-Y_{t}^{t, x}\right|^{2+\varepsilon^{\prime}}\right] \\
& \leq C \mathbb{E}^{\mathbb{P}_{0}^{W}}\left[\left|u\left(t^{\prime}, x\right)-u\left(t^{\prime}, B_{t^{\prime}}^{t, x}\right)\right|^{2+\varepsilon^{\prime}}\right]+C\left|\mathbb{E}^{\mathbb{P}_{0}^{W}}\left[Y_{t^{\prime}}^{t, x}-Y_{t}^{t, x}\right]\right|^{2+\varepsilon^{\prime}} \\
& \left.\leq C \mathbb{E}^{\mathbb{P}_{0}^{W}}\left|x-B_{t^{\prime}}^{t, x}\right|^{2+\varepsilon^{\prime}}\right]+C\left|\mathbb{E}^{\mathbb{P}_{0}^{W}}\left[\int_{t}^{t^{\prime}} f\left(r, B_{r}^{t, x}, Y_{r}^{t, x}, Z_{r}^{t, x}, \widehat{a}_{r}\right) d r+\int_{t}^{t^{\prime}} d K_{r}^{t, x}\right]\right|^{2+\varepsilon^{\prime}} \\
& \leq C\left|t-t^{\prime}\right|^{1+\frac{\varepsilon^{\prime}}{2}}+C \sup _{\mathbb{P} \in \mathcal{P}^{t}} \mathbb{E}_{0}^{\mathbb{P}_{0}^{W}}\left[\left(\int_{t}^{t^{\prime}}\left|f\left(r, B_{r}^{t, x}, Y_{r}^{t, x}, Z_{r}^{t, x}, \widehat{a}_{r}\right)\right| d r\right)^{2+\varepsilon^{\prime}}+\left(\int_{t}^{t^{\prime}} d K_{r}^{t, x}\right)^{2+\varepsilon^{\prime}}\right] \\
& \leq C\left|t-t^{\prime}\right|^{1+\frac{\varepsilon^{\prime}}{2}}\left(1+\left\|Y^{t, x}\right\|_{\mathbb{D}^{2+\varepsilon^{\prime}}}^{1+\frac{\varepsilon^{\prime}}{2}}+\left\|Z^{t, x}\right\|_{\mathbb{H}^{2+\varepsilon^{\prime}}}^{1+\frac{\varepsilon^{\prime}}{2}}+\sup _{\mathbb{P} \in \mathbb{P}^{t}}^{\mathbb{P}_{0}^{W}}\left[\left(K_{T}^{t, x}+\int_{0}^{T}\left|B_{s}^{t, x}\right| d s\right)^{2+\varepsilon^{\prime}}\right]\right) \\
& \leq C\left|t-t^{\prime}\right|^{1+\frac{\varepsilon^{\prime}}{2}} .
\end{aligned}
$$

By Kolmogorov-Chentsov Theorem, this implies immediately that a $\mathbb{P}_{0}^{W}$-version of $u$ is $\eta$-Hölder continuous in $t$, for any $\eta \in(0, \varepsilon /(2(2+\varepsilon)))$.

\subsubsection{Stochastic flow and definitions}

We follow Buckdahn and Ma [9]. The definition of our stochastic viscosity solution will depend on the following stochastic flow $\eta \in C\left(\mathbb{F}^{W},[0, T] \times \mathbb{R}^{d} \times \mathbb{R}\right)$, defined as the unique solution of the (SDE)

$$
\eta(t, x, y)=y+\int_{t}^{T} g(s, x, \eta(s, x, y)) \circ d \overleftarrow{W}_{s}, 0 \leq t \leq T
$$

Under Assumption 5.1, for fixed $x$ the random field $\eta(., x,$.$) is continuously differentiable in the variable y$, and the mapping $y \longmapsto \eta(t, x, y, \omega)$ defines a diffeomorphism for all $(t, x), \mathbb{P}-$ a.s.

We denote by $\mathcal{E}(t, x, y)$ the $y$-inverse of $\eta(t, x, y)$, so $\mathcal{E}(t, x, y)$ is the solution of the following first-order SPDE

$$
\mathcal{E}(t, x, y)=y-\int_{t}^{T} D_{y} \mathcal{E}(s, x, y) g(s, x, y) \circ d \overleftarrow{W}_{s}, \forall(t, x, y), \mathbb{P}-a . s .
$$

We note that $\mathcal{E}(t, x, \eta(t, x, y))=\mathcal{E}(T, x, \eta(T, x, y))=y, \forall(t, x, y)$. We now define the notion of stochastic viscosity solution for SPDE (5.1). 
Definition 5.2. (i) A random field $u \in C\left(\mathbb{F}^{W},[0, T] \times \mathbb{R}^{d}\right)$ is called a stochastic viscosity subsolution (resp. supersolution) of SPDE (5.8), if

$$
u(T, x) \leq(r e s p . \geq) \phi(x), \forall x \in \mathbb{R}^{d},
$$

and if for any $\tau \in \mathcal{M}_{0, T}^{W}, \zeta \in L^{0}\left(\mathcal{F}_{\tau, T}^{W} ; \mathbb{R}^{d}\right)$, and any random field $\varphi \in C^{1,2}\left(\mathcal{F}_{\tau, T}^{W},[0, T] \times \mathbb{R}^{d}\right)$ satisfying

$$
u(t, x)-\eta(t, x, \varphi(t, x)) \leq(\text { resp. } \geq) 0=u(\tau, \zeta)-\eta(\tau, \zeta, \varphi(\tau, \zeta),
$$

for all $(t, x)$ in a neighborhood of $(\tau, \zeta), \mathbb{P}_{W}^{0}-$ a.e. on the set $\{0<\tau<T\}$, it holds that

$$
-\hat{h}\left(\tau, \zeta, \psi(\tau, \zeta), D \psi(\tau, \zeta), D^{2} \psi(\tau, \zeta)\right) \leq(\operatorname{resp} . \geq) D_{y} \eta(\tau, \zeta, \varphi(\tau, \zeta)) D_{t} \varphi(\tau, \zeta),
$$

$\mathbb{P}$ - a.e. on $\{0<\tau<T\}$, where $\psi(t, x):=\eta(t, x, \varphi(t, x))$.

(ii) A random field $u \in C\left(\mathbb{F}^{W},[0, T] \times \mathbb{R}^{d}\right)$ is called a stochastic viscosity solution of SPDE (5.8), if it is both a stochastic viscosity subsolution and a supersolution.

Definition 5.3. A random field $u \in C\left(\mathbb{F}^{W},[0, T] \times \mathbb{R}^{d}\right)$ is called a $\omega$-wise viscosity (sub-, super-) solution, if for $\mathbb{P}$ - a.e. $\omega \in \Omega, u(\omega, \cdot)$ is a (deterministic) viscosity (sub-, super-) solution of the SPDE (5.8).

Remark 5.1. If we assume that $\varphi \in C^{1,2}\left(\mathbb{F}^{W},[0, T] \times \mathbb{R}^{d}\right)$, and that $g \in C^{0,0,3}\left([0, T] \times \mathbb{R}^{d} \times \mathbb{R} ; \mathbb{R}^{l}\right)$, then a straightforward computation using the Itô-Ventzell formula shows that the random field $\psi(t, x)=\eta(t, x, \varphi(t, x))$ satisfies

$$
d \psi(t, x)=D_{y} \eta(t, x, \varphi(t, x)) D_{t} \varphi(t, x) d t+g(t, x, \psi(t, x)) \circ d \overleftarrow{W}_{t}, t \in[0, T]
$$

Since $g(\tau, \zeta, \psi(\tau, \zeta))=g(\tau, \zeta, u(\tau, \zeta))$ by defintion, it seems natural to compare

$$
\hat{h}\left(\tau, \zeta, \psi(\tau, \zeta), D \psi(\tau, \zeta), D^{2} \psi(\tau, \zeta)\right) \text {, with } D_{y} \eta(\tau, \zeta, \varphi(\tau, \zeta)) D_{t} \varphi(\tau, \zeta),
$$

to characterize a viscosity solution of SPDE $(\hat{h}, g)$.

If the function $g \equiv 0$ in $S P D E$ (5.1), the flow $\eta$ becomes $\eta(t, x, y)=y, \forall(t, x, y)$ and $\psi(t, x)=\varphi(t, x)$. Thus the definition of a stochastic viscosity solution becomes the same as that of a deterministic viscosity solution (see, e.g. Crandall, Ishii and Lions [18]).

One of the main results of our paper is the following probabilistic representation of stochastic viscosity solution for fully nonlinear SPDEs, which is, to the best of our knowledge, the first result of this kind for such a class of SPDEs. The proof will be obtained in the subsequent subsections.

Theorem 5.3. Let Assumptions 5.1, 5.2 and 5.3 hold true and $\left(Y_{s}^{t, x}, Z_{s}^{t, x}, K_{s}^{t, x}\right)$ be the unique solution of the 2BDSDE (5.4). Then,

$$
u(t, x)=Y_{t}^{t, x}=\sup _{\mathbb{P} \in \mathcal{P}^{t}} y_{t}^{\mathbb{P}, t, x}\left(T, \phi\left(B_{T}^{t, x}\right)\right), \mathbb{P}_{W}^{0}-\text { a.s, for all }(t, x) \in[0, T] \times \mathbb{R}^{d},
$$

is a stochastic viscosity solution of SPDE (5.8). Moreover,

$$
u(t, x)=\eta(t, x, v(t, x)), v(t, x)=\mathcal{E}(t, x, u(t, x)), \mathbb{P}_{W}^{0}-a . s ., \text { and } v(t, x)=U_{t}^{t, x},
$$

where $\left(U^{t, x}, V^{t, x}, \tilde{K}^{t, x}\right)$ is a solution of the following $2 B S D E$, for all $t \leq s \leq T$,

$$
U_{s}^{t, x}=\phi\left(B_{T}^{t, x}\right)-\int_{s}^{T} \tilde{f}\left(r, B_{r}^{t, x}, Y_{r}^{t, x}, Z_{r}^{t, x}, \widehat{a}_{r}\right) d r-\int_{s}^{T} V_{r}^{t, x} \cdot d B_{r}+\tilde{K}_{T}^{t, x}-\tilde{K}_{s}^{t, x},
$$


with $\tilde{f}:[0, T] \times \mathbb{R}^{d} \times \mathbb{R} \times \mathbb{R}^{d} \times D_{f} \longrightarrow \mathbb{R}$ defined by

$$
\begin{aligned}
\tilde{f}(t, x, y, z, a):= & \frac{1}{D_{y} \eta(t, x, y)}\left(f\left(t, x, \eta(t, x, y), D_{y} \eta(t, x, y) z+D_{x} \eta(t, x, y), a\right)-\frac{1}{2} \operatorname{Tr}\left[a D_{x x} \eta(t, x, y)\right]\right. \\
& \left.-z^{\top} a D_{x y} \eta(t, x, y)-\frac{1}{2} \operatorname{Tr}\left[D_{y y} \eta(t, x, y) a^{1 / 2} z z^{\top} a^{1 / 2}\right]\right) .
\end{aligned}
$$

\subsubsection{Doss-Sussmann transformation}

In this subsection, we use the so-called Doss-Sussmann transformation to convert the fully nonlinear SPDEs (5.8) to PDEs with random coefficients. This transformation permits to remove the martingale term from the SPDEs. To begin with, let us note that, under Assumption 5.1 (iii), the random field $\eta \in C^{0,2,2}\left(\mathbb{F}^{W},[0, T] \times\right.$ $\mathbb{R}^{d} \times \mathbb{R}$ ), thus so is $\mathcal{E}$. Now for any random field $\psi:[0, T] \times \mathbb{R}^{d} \times \Omega \longrightarrow \mathbb{R}$, consider the transformation introduced in Definition 5.2

$$
\varphi(t, x)=\mathcal{E}(t, x, \psi(t, x)), \quad(t, x) \in[0, T] \times \mathbb{R}^{d},
$$

or equivalently, $\psi(t, x)=\eta(t, x, \varphi(t, x))$. One can easily check that $\psi \in C^{0, p}\left(\mathbb{F}^{W},[0, T] \times \mathbb{R}^{d}\right)$ if and only if $\varphi \in C^{0, p}\left(\mathbb{F}^{W},[0, T] \times \mathbb{R}^{d}\right)$, for $p=0,1,2$. Moreover, if $\varphi \in C^{0,2}\left(\mathcal{F}^{W},[0, T] \times \mathbb{R}^{d}\right)$, then

$$
\begin{gathered}
D_{x} \psi=D_{x} \eta+D_{y} \eta D_{x} \varphi \\
D_{x x} \psi=D_{x x} \eta+2\left(D_{x y} \eta\right)\left(D_{x} \varphi\right)^{\top}+\left(D_{y y} \eta\right)\left(D_{x} \varphi\right)\left(D_{x} \varphi\right)^{\top}+\left(D_{y} \eta\right)\left(D_{x x} \varphi\right) .
\end{gathered}
$$

Furthermore, since $\mathcal{E}(t, x, \eta(t, x, y)) \equiv y, \forall(t, x, y), \mathbb{P}-a . s$., differentiating the equation up to the second order we have (suppressing variables fro simplicity), for all $(t, x, y)$ and $\mathbb{P}-$ a.s.,

$$
\begin{aligned}
& D_{x} \mathcal{E}+D_{y} \mathcal{E} D_{x} \eta=0, D_{y} \mathcal{E} D_{y} \eta=1, \\
& D_{x x} \mathcal{E}+2\left(D_{x y} \mathcal{E}\right)\left(D_{x} \eta\right)^{\top}+\left(D_{y y} \mathcal{E}\right)\left(D_{x} \eta\right)\left(D_{x} \eta\right)^{\top}+\left(D_{y} \mathcal{E}\right)\left(D_{x x} \eta\right)=0, \\
& \left(D_{x y} \mathcal{E}\right)\left(D_{y} \eta\right)+\left(D_{y y} \mathcal{E}\right)\left(D_{x} \eta\right)\left(D_{y} \eta\right)+\left(D_{y} \mathcal{E}\right)\left(D_{x y} \eta\right)=0 \\
& \left(D_{y y} \mathcal{E}\right)\left(D_{y} \eta\right)^{2}+\left(D_{y} \mathcal{E}\right)\left(D_{y y} \eta\right)=0 .
\end{aligned}
$$

The following additional assumption is needed to study the growth of the random fields $\eta$ and $\mathcal{E}$ (see [9], p.188-189).

Assumption 5.3. For any $\varepsilon>0$, there exists a function $G^{\varepsilon} \in C^{1,2,2,2}\left([0, T] \times \mathbb{R}^{n} \times \mathbb{R}^{d} \times \mathbb{R}\right)$, such that

$$
\frac{\partial G^{\varepsilon}}{\partial t}(t, w, x, y)=\varepsilon, \frac{\partial G^{\varepsilon}}{\partial w^{i}}=g^{i}\left(t, x, G^{\varepsilon}(t, w, x, y)\right), i=1, \cdots, n, \text { and } G^{\varepsilon}(0,0, x, y)=y .
$$

Proposition 5.1. Let $\eta$ be the unique solution to $S D E$ (5.10) and $\mathcal{E}$ be the $y$-inverse of $\eta$ (the solution to (5.11)). Then, under Assumption 5.3, there exists a constant $C>0$, depending only on the bound of $g$ and its partial derivatives, such that for $\zeta=\eta, \mathcal{E}$, it holds for all $(t, x, y)$ and $\mathbb{P}_{0}^{W}-$ a.s. that

$$
\begin{aligned}
& |\zeta(t, x, y) \leq| y|+C| W_{t} \mid, \\
& \left|D_{x} \zeta\right|+\left|D_{y} \zeta\right|+\left|D_{x x} \zeta\right|+\left|D_{x y} \zeta\right|+\left|D_{y y} \zeta\right| \leq C \exp \left(C\left|W_{t}\right|\right),
\end{aligned}
$$

where all the derivatives are evaluated at $(t, x, y)$.

The proof of this proposition is done in [9], p.189-191, so we omit it. Now, we will use the Doss transformation to transform SPDE (5.1) to PDE with random coefficients and we obtain the following proposition where the proof follows the lines of the proof of Proposition 3.1. in [9] (p. 187-188). 
Proposition 5.2. Let Assumptions 5.1, 5.2 and 5.3 hold true. A random field $u$ is a stochastic viscosity sub(resp. super-) solution to SPDE (5.8) if and only if $v(.,)=.\mathcal{E}(., ., u(.,)$.$) is a stochastic viscosity solution to the$ following PDE with random coefficients

$$
\left\{\begin{array}{l}
d v(t, x)+\tilde{h}\left(t, x, v(t, x), D v(t, x), D^{2} v(t, x)\right) d t=0, \\
v(T, x)=\phi(x)
\end{array}\right.
$$

with

$$
\tilde{h}(t, x, y, z, \gamma):=\sup _{a \in \mathbb{S}_{d}^{>0}}\left\{\frac{1}{2} \operatorname{Tr}[a \gamma]-\tilde{f}(t, x, y, z, a)\right\}+\frac{1}{2} \operatorname{Tr}\left[g(s, x, \eta(s, x, y)) D_{y} g(s, x, \eta(s, x, y))^{\top}\right] .
$$

Consequently, $u$ is a stochastic viscosity solution of SPDE (5.8) if and only if $v(.,)=.\mathcal{E}(., ., u(.,)$.$) is a stochastic$ viscosity solution to the PDE with random coefficients (5.17).

Proof. Let $u \in C\left(\mathbb{F}^{W},[0, T] \times \mathbb{R}^{d}\right)$ be a stochastic viscosity subsolution of SPDE (5.8) and let $v$ be defined by $v(t, x)=\mathcal{E}(t, x, u(t, x))$. In order to show that $v$ is a stochastic viscosity subsolution to the PDE (5.17), we let $\tau \in \mathcal{M}_{0, T}^{W}, \zeta \in L^{0}\left(\mathcal{F}_{\tau, T}^{W} ; \mathbb{R}^{d}\right)$ be arbitrary given, and let $\varphi \in C^{1,2}\left(\mathcal{F}_{\tau, T}^{W},[0, T] \times \mathbb{R}^{d}\right)$ be such that

$$
v(t, x)-\varphi(t, x) \leq 0=v(\tau, \zeta)-\varphi(\tau, \zeta),
$$

for all $(t, x)$ in a neighborhood of $(\tau, \zeta), \mathbb{P}_{W}^{0}-$ a.e. on the set $\{0<\tau<T\}$.

Now, we define $\psi(t, x)=\eta(t, x, \varphi(t, x)), \forall(t, x) \mathbb{P}_{W}^{0}-$ a.e. Since $y \longmapsto \eta(t, x)$ is strictly increasing, we have

$$
\begin{aligned}
u(t, x)-\psi(t, x) & =\eta(t, x, v(t, x))-\eta(t, x, \varphi(t, x)) \\
& \leq 0=\eta(\tau, \zeta, v(\tau, \zeta))-\eta(\tau, \zeta, \varphi(\tau, \zeta))=u(\tau, \zeta)-\psi(\tau, \zeta),
\end{aligned}
$$

for all $(t, x)$ in a neighborhood of $(\tau, \zeta), \mathbb{P}_{W}^{0}-$ a.e. on the set $\{0<\tau<T\}$. Then, since $u$ is a stochastic viscosity subsolution of $\operatorname{SPDE}(5.8)$, we have $\mathbb{P}_{W}^{0}-$ a.e. on $\{0<\tau<T\}$,

$$
-\hat{h}\left(\tau, \zeta, \psi(\tau, \zeta), D \psi(\tau, \zeta), D^{2} \psi(\tau, \zeta)\right) \leq D_{y} \eta(\tau, \zeta, \varphi(\tau, \zeta)) D_{t} \varphi(\tau, \zeta)
$$

On the other hand, we recall the expression of $\hat{h}$,

$$
\begin{aligned}
\hat{h}(t, x, y, z, \gamma) & :=\sup _{a \in \mathbb{S}_{d}^{>0}}\left\{\frac{1}{2} \operatorname{Tr}[a \gamma]-F(t, x, y, z, a)\right\} \\
& =\sup _{a \in \mathbb{S}_{d}^{>0}}\left\{\frac{1}{2} \operatorname{Tr}[a \gamma]-f\left(s, x, y, z, \widehat{a}_{s}\right)+\frac{1}{2} \operatorname{Tr}\left[g(s, x, y) D_{y} g(s, x, y)^{\top}\right] .\right\}
\end{aligned}
$$

Thus, using (5.15), we have

$$
\begin{aligned}
\operatorname{Tr}\left[a D^{2} \psi(\tau, \zeta)\right]= & \operatorname{Tr}\left[a D_{x x} \eta(\tau, \zeta, \varphi(\tau, \zeta))\right]+\operatorname{Tr}\left[\left(D_{x} \varphi(\tau, \zeta)\right)^{\top} a\left(D_{x y} \eta(\tau, \zeta, \varphi(\tau, \zeta))\right]\right. \\
& +\operatorname{Tr}\left[a\left(D_{y y} \eta(\tau, \zeta, \varphi(\tau, \zeta))\right)\left(D_{x} \varphi(\tau, \zeta)\right)\left(D_{x} \varphi(\tau, \zeta)\right)^{\top}\right]+\operatorname{Tr}\left[a\left(D_{y} \eta(\tau, \zeta, \varphi(\tau, \zeta))\right)\left(D_{x x} \varphi(\tau, \zeta)\right)\right],
\end{aligned}
$$

Finally, plugging the above calculations in (5.19) and appealing to (5.14), we conclude that

$$
-\tilde{h}\left(\tau, \zeta, \varphi(\tau, \zeta), D \varphi(\tau, \zeta), D^{2} \varphi(\tau, \zeta)\right) \leq D_{t} \varphi(\tau, \zeta)
$$

which is the desired result. The reciprocal part of the proposition can be proved in a similar way. 
We apply now Doss-Sussmann transformation for the 2BDSDE (5.4) so that the Stratonovich backward integral vanishes. Thus, the 2BDSDE will become a 2BSDE (a standard one) with a new generator $\tilde{f}$ (5.14), which is quadratic in $z$. A similar class of 2BSDEs has been studied by Possamaï and Zhou [59] and Lin [41] in the case of a bounded final condition $\phi\left(B_{T}^{t, x}\right)$ and a generator $F$ satisfying (see Assumption 2.1. (iv) p. 3776 in [59])

$$
\left|F_{t}(x, y, z, a)\right| \leq \alpha+\beta|y|+\frac{\gamma}{2}\left|a^{1 / 2} z\right|^{2},
$$

for some positive constants $\alpha, \beta$ and $\gamma$. In our case, for fixed $\omega^{W}$, thanks to Proposition 5.1, we know that the generator $\tilde{f}$ satisfies a similar assumption, so that we can apply the results of [59] or [41].

Let us then define the following three processes

$$
\begin{aligned}
U_{s}^{t, x} & :=\mathcal{E}\left(t, B_{s}^{t, x}, Y_{s}^{t, x}\right), \\
V_{s}^{t, x} & :=D_{y} \mathcal{E}\left(s, B_{s}^{t, x}, Y_{t}^{t, x}\right) Z_{s}^{t, x}+D_{x} \mathcal{E}\left(s, B_{s}^{t, x}, Y_{s}^{t, x}\right), \\
\tilde{K}_{s}^{t, x} & :=\int_{0}^{s} D_{y} \mathcal{E}\left(r, B_{r}^{t, x}, Y_{r}^{t, x}\right) d K_{r}^{t, x} .
\end{aligned}
$$

Theorem 5.4. Let Assumptions 5.1, 5.2 and 5.3 hold true. Then $\left(U^{t, x}, V^{t, x}, \tilde{K}^{t, x}\right)$ is the unique solution of the $2 B S D E$ (5.13).

Proof. It is easily checked that the mapping $(B, Y, Z, K) \longmapsto(B, U, V, \tilde{K})$ is one-to-one, and admits as an inverse

$$
Y_{t}=\eta\left(t, B_{t}, U_{t}\right), Z_{t}=D_{y} \eta\left(t, B_{t}, U_{t}\right) V_{t}+D_{x} \eta\left(t, B_{t}, U_{t}\right), K_{t}=\int_{0}^{t} D_{y} \eta\left(s, B_{s}, U_{s}\right) d \tilde{K}_{s} .
$$

Consequently, the uniqueness of (5.13) follows from that of 2BDSDE (5.4), thanks to (5.21) and (5.22). Thus we need only show that $(U, V, \tilde{K})$ is a solution of the 2BSDE (5.13). Applying the generalized Itô-Ventzell formula (see Lemma A.2 below) to $\mathcal{E}\left(t, B_{t}, Y_{t}\right)$, one derives that for any $(t, x) \in[0, T] \times \mathbb{R}^{d}$

$$
\begin{aligned}
U_{t}=\mathcal{E}\left(t, B_{t}, Y_{t}\right)= & \phi\left(B_{T}\right)-\int_{t}^{T} D_{y} \mathcal{E}\left(s, B_{s}, Y_{s}\right) f\left(s, B_{s}, Y_{s}, Z_{s}, \widehat{a}_{s}\right) d s \\
& -\int_{t}^{T} D_{x} \mathcal{E}\left(s, B_{s}, Y_{s}\right) \cdot d B_{s}-\int_{t}^{T} D_{y} \mathcal{E}\left(s, B_{s}, Y_{s}\right) Z_{s} \cdot d B_{s}+\int_{t}^{T} D_{y} \mathcal{E}\left(s, B_{s}, Y_{s}\right) d K_{s} \\
& -\frac{1}{2} \int_{t}^{T} \operatorname{Tr}\left[D_{x x} \mathcal{E}\left(s, B_{s}, Y_{s}\right) \widehat{a}_{s}\right] d s-\frac{1}{2} \int_{t}^{T} \operatorname{Tr}\left[D_{y y} \mathcal{E}\left(s, B_{s}, Y_{s}\right) \widehat{a}_{s}^{1 / 2} Z_{s} Z_{s}^{\top} \widehat{a}_{s}^{1 / 2}\right] d s \\
& -\int_{t}^{T} \operatorname{Tr}\left[D_{x y} \mathcal{E}\left(s, B_{s}, Y_{s}\right) Z_{s}^{\top} \widehat{a}_{s}\right] d s \\
= & \phi\left(B_{T}\right)-\int_{t}^{T} \mathcal{H}\left(s, B_{s}, Y_{s}, Z_{s}, \widehat{a}_{s}\right) d s-\int_{t}^{T} V_{s} \cdot d B_{s}+\tilde{K}_{T}-\tilde{K}_{t},
\end{aligned}
$$

where

$$
\mathcal{H}(s, x, y, z, a):=\left(D_{y} \mathcal{E}\right) f(s, x, y, z, a)+\frac{1}{2} \operatorname{Tr}\left[\left(D_{x x} \mathcal{E}\right) a\right]+\frac{1}{2} \operatorname{Tr}\left[\left(D_{y y} \mathcal{E}\right) a^{1 / 2} z z^{\top} a^{1 / 2}\right]+\operatorname{Tr}\left[\left(D_{x y} \mathcal{E}\right) z^{\top} a\right] .
$$

Next, we can show that

$$
\mathcal{H}\left(s, B_{s}, Y_{s}, Z_{s}, \widehat{a}_{s}\right)=\tilde{f}\left(s, B_{s}, U_{s}, V_{s}, \widehat{a}_{s}\right), \forall s \in[0, T], \mathbb{P}-a . s .
$$

similarly as done in Buckdahn and Ma [9] (proof of Theorem 5.1. page 198-199). 
The process $\tilde{K}$ is a non-decreasing process thanks to the fact that $y \longmapsto \eta(t, x, y)$ is strictly increasing and the non-decreasing of $K$. Now, it remains to prove the minimum condition (2.3) for the process $\tilde{K}$. Notice that by Proposition 5.1, and since $\widehat{a}$ is bounded under any of the measure we consider, it is clear that $D_{y} \mathcal{E}\left(r, B_{r}^{t, x}, Y_{r}^{t, x}\right)$ has moments of any order under any $\mathbb{P}$. Therefore, we can argue exactly as in Step (ii) of the proof of Theorem 3.1 to show that $\tilde{K}$ inherits the required minimality condition directly from $K$.

We are now ready for the proof of our main theorem

Proof. [Proof of Theorem 5.3] First, we introduce the random field $v(t, x)=U_{t}^{t, x}$, where $U$ is the solution of $2 \operatorname{BSDE}$ (5.13). Then by (5.21) and (5.22) we know that, for $(t, x) \in[0, T] \times \mathbb{R}^{d}$

$$
u(t, x)=\eta(t, x, v(t, x)), v(t, x)=\mathcal{E}(t, x, u(t, x)) .
$$

Thanks to Proposition 5.1, we know that we only need to prove that the random field $v$ defined in (5.24) is a viscosity solution of the PDE with random coefficients (5.17). The idea is then to follow the proof in [59, Theorem 7.3], which itself follows [65, Theorem 5.11], to prove that the solution of 2BSDE (5.13) $v(t, x)=U_{t}^{t, x}$ is an $\omega$-wise viscosity solution of the PDE (5.17) (recall Definition 5.3), which then ends the proof. It suffices to

notice that the fact that $f$ satisfies Assumptions 5.1 and Assumption 5.1 implies that, for fixed $\omega^{W}, \tilde{f}$ satisfies Assumption 7.1 of [59].

\section{Appendix A: Technical results}

\section{A.1. Itô and Itô-Ventzell formulae}

The following Itô's formula is a mix between the classical forward and backward Itô's formulas and is similar to Lemma 1.3 in [52]. We give it here for ease of reference and completeness. The proof being standard, we omit it.

Lemma A.1. Let $X^{1}$ and $X^{2}$ be defined, for $i=1,2$, by

$$
X_{t}^{i}=X_{0}^{i}+\int_{0}^{t} \alpha_{s}^{i} d s+\int_{0}^{t} \beta_{s}^{i} \cdot d B_{s}+\int_{0}^{t} \gamma_{s}^{i} \cdot d \overleftarrow{W}_{s}+K_{t}^{i}, 0 \leq t \leq T, \mathbb{P}-a . s .
$$

for some càdlàg bounded variation and $\mathbb{G}$-progressively measurable processes $K^{i}$, such that one of them is continuous. We then have

$$
\begin{aligned}
X_{t}^{1} X_{t}^{2}= & X_{0}^{1} X_{0}^{2}+\int_{0}^{t}\left(\widehat{a}_{s}^{1 / 2} \beta_{s}^{1} \cdot \widehat{a}_{s}^{1 / 2} \beta_{s}^{2}-\gamma_{s}^{1} \cdot \gamma_{s}^{2}+\alpha_{s}^{1} X_{s}^{2}+\alpha_{s}^{2} X_{s}^{1}\right) d s+\int_{0}^{t}\left(X_{s}^{2} \beta_{s}^{1}+X_{s}^{1} \beta_{s}^{2}\right) \cdot d B_{s} \\
& +\int_{0}^{t}\left(X_{s}^{1} \gamma_{s}^{2}+X_{s}^{2} \gamma_{s}^{1}\right) \cdot d \overleftarrow{W}_{s}+\int_{0}^{t} X_{s^{-}}^{1} d K_{s}^{2}+\int_{0}^{t} X_{s^{-}}^{2} d K_{s}^{1}, t \in[0, T], \mathbb{P}-a . s .
\end{aligned}
$$

We now give a generalized version of Itô-Ventzell formula that combines the generalized Itô formula of Pardoux and Peng [52] and the Itô-Ventzell formula of Ocone and Pardoux [49].

Lemma A.2. (Generalized Itô-Ventzell formula)

Suppose that $F \in C^{0,2}\left(\mathbb{F},[0, T] \times \mathbb{R}^{k}\right)$ is a semimartingale with spatial parameter $x \in \mathbb{R}^{k}$ :

$$
F(t, x)=F(0, t)+\int_{0}^{t} G(s, x) d s+\int_{0}^{t} H(s, x) \cdot d B_{s}+\int_{0}^{t} K(s, x) \cdot d \overleftarrow{W}_{s}, \quad t \in[0, T]
$$


where $G \in C^{0,2}\left(\mathbb{F}^{B},[0, T] \times \mathbb{R}^{k}\right), H \in C^{0,2}\left(\mathbb{F}^{B},[0, T] \times \mathbb{R}^{k} ; \mathbb{R}^{d}\right)$ and $K \in C^{0,2}\left(\mathbb{F}^{W},[0, T] \times \mathbb{R}^{k} ; \mathbb{R}^{l}\right)$. Let $\phi \in$ $C\left(\mathbb{F},[0, T] ; \mathbb{R}^{k}\right)$ be a process of the form

$$
\phi_{t}=\phi_{0}+A_{t}+\int_{0}^{t} \gamma_{s} \cdot d B_{s}+\int_{0}^{t} \delta_{s} \cdot d \overleftarrow{W}_{s}, \quad t \in[0, T]
$$

where $\gamma \in \mathbb{H}_{k \times d}^{2}, \delta \in \mathbb{H}_{k \times l}^{2}$ and $A$ is a continuous $\mathbb{F}$-adapted process with paths of locally bounded variation. Then, $\mathbb{P}$-almost surely, it holds for all $0 \leq t \leq T$ that

$$
\begin{aligned}
F\left(t, \phi_{t}\right)= & F(0, x)+\int_{0}^{t} G\left(s, \phi_{s}\right) d s+\int_{0}^{t} H\left(s, \phi_{s}\right) \cdot d B_{s}+\int_{0}^{t} K\left(s, \phi_{s}\right) \cdot d \overleftarrow{W}_{s} \\
& +\int_{0}^{t} D_{x} F\left(s, \phi_{s}\right) d A_{s}+\int_{0}^{t} D_{x} F\left(s, \phi_{s}\right) \gamma_{s} \cdot d B_{s}+\int_{0}^{t} D_{x} F\left(s, \phi_{s}\right) \delta_{s} \cdot d \overleftarrow{W}_{s} \\
& +\frac{1}{2} \int_{0}^{t} \operatorname{Tr}\left(D_{x x} F\left(s, \phi_{s}\right) \gamma_{s} \gamma_{s}^{\top}\right) d s-\frac{1}{2} \int_{0}^{t} \operatorname{Tr}\left[D_{x x} F\left(s, \phi_{s}\right) \delta_{s} \delta_{s}^{\top}\right] d s \\
& +\int_{0}^{t} \operatorname{Tr}\left(D_{x} H\left(s, \phi_{s}\right) \gamma_{s}^{\top}\right) d s-\int_{0}^{t} \operatorname{Tr}\left[D_{x} F\left(s, \phi_{s}\right) \delta_{s}^{\top}\right] d s .
\end{aligned}
$$

\section{A.2. Proof of Lemma 4.1}

We divide the proof in two steps.

Step 1: We start by showing the result in the case where $F$ and $g$ do not depend on $(y, z)$. In this case, we can solve directly the BDSDEs to find that for $\mathbb{P}-$ a.e. $\left(\omega^{B}, \omega^{W}\right) \in \Omega$

$$
y_{t}^{\mathbb{P}}\left(\omega^{B}, \omega^{W}\right)=\mathbb{E}^{\mathbb{P}}\left[\xi+\int_{t}^{T} \widehat{F}_{s} d s+\int_{t}^{T} g_{s} \cdot d \overleftarrow{W}_{s} \mid \mathcal{G}_{t}\right]\left(\omega^{B}, \omega^{W}\right) .
$$

Then, since $\xi$ is actually $\mathcal{F}_{T}^{B}$-measurable, we deduce immediately, using the definition of the r.c.p.d. that for $\mathbb{P}_{0}^{W}$-a.e. $\omega^{W} \in \Omega^{W}$

$$
\mathbb{E}^{\mathbb{P}}\left[\xi \mid \mathcal{G}_{t}\right]\left(\omega^{B}, \omega^{W}\right)=\mathbb{E}^{\mathbb{P}_{B}\left(\omega^{W}\right)}\left[\xi \mid \mathcal{F}_{t}^{B}\right]\left(\omega^{B}\right)=\mathbb{E}^{\mathbb{P}_{B}^{t, \omega^{B}}\left(\omega^{W}\right)}\left[\xi^{t, \omega^{B}}\right] .
$$

Next, we know from the results of Stricker and Yor [66] that we can define a measurable map from $\left(\Omega^{W} \times\right.$ $\left.[0, T], \mathcal{F}_{T} \otimes \mathcal{B}([0, T])\right)$ to $(\mathbb{R}, \mathcal{B}(\mathbb{R}))$ which coincides $\mathbb{P}_{W}^{0} \otimes d t$-a.e. with the conditional expectation of $g_{s}$, under $\mathbb{P}^{B}\left(\cdot ; \omega^{W}\right)$ (remember that this is a stochastic kernel, and thus measurable), with respect to the $\sigma$-algebra $\mathcal{F}_{t}^{B}$. For notational simplicity, we still denote this map as

$$
\left(\omega^{W}, s\right) \longmapsto \mathbb{E}^{\mathbb{P}_{B}\left(\cdot ; \omega^{W}\right)}\left[g_{s}\left(\cdot, \omega^{W}\right) \mid \mathcal{F}_{t}^{B}\right] .
$$

In other words, the above map does indeed define a stochastic process. That being said, we claim that for $\mathbb{P}-$ a.e. $\omega \in \Omega$

$$
\begin{aligned}
\mathbb{E}^{\mathbb{P}}\left[\int_{t}^{T} g_{s} \cdot d \overleftarrow{W}_{s} \mid \mathcal{G}_{t}\right]\left(\omega^{B}, \omega^{W}\right) & =\left(\int_{t}^{T} \mathbb{E}^{\mathbb{P}_{B}(\cdot ; \cdot)}\left[g_{s} \mid \mathcal{F}_{t}^{B}\right]\left(\omega^{B}, \cdot\right) \cdot d \overleftarrow{W}_{s}\right)\left(\omega^{W}\right) \\
& =\left(\int_{t}^{T} \mathbb{E}^{\mathbb{P}_{B}^{t, \omega}(\cdot)}\left[g_{s}^{t, \omega^{B}}\right](\cdot) \cdot d \overleftarrow{W}_{s}\right)\left(\omega^{W}\right) .
\end{aligned}
$$

To prove the claim, let us first show it in the case where $g$ is a simple process with the following decomposition

$$
g_{t}\left(\omega^{B}, \omega^{W}\right)=\sum_{i=0}^{n-1} g_{t_{i}}\left(\omega^{B}, \omega^{W}\right) \mathbf{1}_{\left(t_{i}, t_{i+1}\right]}(t)
$$


Then, we have by definition of backward stochastic integrals, for $\mathbb{P}$-a.e. $\left(\omega^{B}, \omega^{W}\right) \in \Omega$

$$
\begin{aligned}
\mathbb{E}^{\mathbb{P}}\left[\int_{t}^{T} g_{s} \cdot d \overleftarrow{W}_{s} \mid \mathcal{G}_{t}\right]\left(\omega^{B}, \omega^{W}\right) & =\sum_{i=0}^{n-1} \mathbb{E}^{\mathbb{P}}\left[g_{t_{i+1}} \cdot\left(W_{t_{i+1} \wedge t}-W_{t_{i} \wedge t}\right) \mid \mathcal{G}_{t}\right]\left(\omega^{B}, \omega^{W}\right) \\
& =\sum_{i=0}^{n-1} \mathbb{E}^{\mathbb{P}}\left[g_{t_{i+1}} \mid \mathcal{G}_{t}\right]\left(\omega^{B}, \omega^{W}\right) \cdot\left(W_{t_{i+1} \wedge t}-W_{t_{i} \wedge t}\right)\left(\omega^{W}\right)
\end{aligned}
$$

Notice next that for $\mathbb{P}$ - a.e. $\left(\omega^{B}, \omega^{W}\right) \in \Omega$

$$
\mathbb{E}^{\mathbb{P}}\left[g_{t_{i+1}} \mid \mathcal{G}_{t}\right]\left(\omega^{B}, \omega^{W}\right)=\mathbb{E}^{\mathbb{P}_{B}\left(\cdot ; \omega^{W}\right)}\left[g_{t_{i+1}}\left(\cdot, \omega^{W}\right) \mid \mathcal{F}_{t}^{B}\right]\left(\omega^{B}\right)
$$

Indeed, for any $X$ which is $\mathcal{G}_{t}$-measurable, we have

$$
\begin{aligned}
& \int_{\Omega} \mathbb{E}^{\mathbb{P}_{B}\left(\cdot ; \omega^{W}\right)}\left[g_{t_{i+1}}\left(\cdot, \omega^{W}\right) \mid \mathcal{F}_{t}^{B}\right]\left(\omega^{B}\right) X\left(\omega^{B}, \omega^{W}\right) d \mathbb{P}_{B}\left(\omega^{B} ; \omega^{W}\right) d \mathbb{P}_{W}^{0}\left(\omega^{W}\right) \\
& =\int_{\Omega^{W}}\left(\int_{\Omega^{B}} \mathbb{E}^{\mathbb{P}_{B}\left(\cdot ; \omega^{W}\right)}\left[g_{t_{i+1}}\left(\cdot, \omega^{W}\right) \mid \mathcal{F}_{t}^{B}\right]\left(\omega^{B}\right) X\left(\omega^{B}, \omega^{W}\right) d \mathbb{P}_{B}\left(\omega^{B} ; \omega^{W}\right)\right) d \mathbb{P}_{W}^{0}\left(\omega^{W}\right) \\
& =\int_{\Omega^{W}}\left(\int_{\Omega^{B}} g_{t_{i+1}}\left(\omega^{B}, \omega^{W}\right) X\left(\omega^{B}, \omega^{W}\right) d \mathbb{P}_{B}\left(\omega^{B} ; \omega^{W}\right)\right) d \mathbb{P}_{W}^{0}\left(\omega^{W}\right) \\
& =\int_{\Omega} g_{t_{i+1}}\left(\omega^{B}, \omega^{W}\right) X\left(\omega^{B}, \omega^{W}\right) d \mathbb{P}\left(\omega^{B}, \omega^{W}\right),
\end{aligned}
$$

where we have used the fact that since for every $\omega^{W} \in \Omega^{W}, \omega^{B} \longmapsto X\left(\omega^{B}, \omega^{W}\right)$ is $\mathcal{F}_{t}^{B}$-measurable, we have by definition of the conditional expectation that

$$
\begin{aligned}
& \int_{\Omega^{B}} \mathbb{E}^{\mathbb{P}_{B}\left(\cdot ; \omega^{W}\right)}\left[g_{t_{i+1}}\left(\cdot, \omega^{W}\right) \mid \mathcal{F}_{t}^{B}\right]\left(\omega^{B}\right) X\left(\omega^{B}, \omega^{W}\right) d \mathbb{P}_{B}\left(\omega^{B} ; \omega^{W}\right) \\
& =\int_{\Omega^{B}} g_{t_{i+1}}\left(\omega^{B}, \omega^{W}\right) X\left(\omega^{B}, \omega^{W}\right) d \mathbb{P}_{B}\left(\omega^{B} ; \omega^{W}\right) .
\end{aligned}
$$

Hence, we deduce finally that

$$
\begin{aligned}
\mathbb{E}^{\mathbb{P}}\left[\int_{t}^{T} g_{s} \cdot d \overleftarrow{W}_{s} \mid \mathcal{G}_{t}\right]\left(\omega^{B}, \omega^{W}\right) & =\sum_{i=0}^{n-1} \mathbb{E}^{\mathbb{P}_{B}\left(\cdot ; \omega^{W}\right)}\left[g_{t_{i+1}}\left(\cdot, \omega^{W}\right) \mid \mathcal{F}_{t}^{B}\right]\left(\omega^{B}\right) \cdot\left(W_{t_{i+1} \wedge t}-W_{t_{i} \wedge t}\right)\left(\omega^{W}\right) \\
& =\left(\int_{t}^{T} \mathbb{E}^{\mathbb{P}_{B}(\cdot ; \cdot)}\left[g_{s} \mid \mathcal{F}_{t}^{B}\right]\left(\omega^{B}, \cdot\right) \cdot d \overleftarrow{W}_{s}\right)\left(\omega^{W}\right)
\end{aligned}
$$

By a simple density argument, we deduce that the same holds for general processes $g$. Next, notice that by definition of r.p.c.d., we have for $\mathbb{P}_{0}^{W}$-a.e. $\omega^{W} \in \Omega^{W}$

$$
\mathbb{E}^{\mathbb{P}_{B}\left(\cdot ; \omega^{W}\right)}\left[g_{s} \mid \mathcal{F}_{t}^{B}\right]\left(\omega^{B}, \omega^{W}\right)=\mathbb{E}^{\mathbb{P}_{B}^{t, \omega^{B}}\left(\cdot ; \omega^{W}\right)}\left[g_{s}^{t, \omega^{B}}\right]\left(\omega^{W}\right) \text {, for } \mathbb{P}_{B}\left(\cdot ; \omega^{W}\right) \text { - a.e. } \omega^{B} \in \Omega^{B} .
$$

By definition of $\mathbb{P}$, we are exactly saying that the above holds for $\mathbb{P}$-a.e. $\omega \in \Omega$. This finally proves (A.3).

Using similar argument, we show that we also have for $\mathbb{P}$-a.e. $\omega \in \Omega$

$$
\mathbb{E}^{\mathbb{P}}\left[\int_{t}^{T} \widehat{F}_{s} d s \mid \mathcal{G}_{t}\right]\left(\omega^{B}, \omega^{W}\right)=\int_{t}^{T} \mathbb{E}^{\mathbb{P}_{B}^{t, \omega^{B}}}\left(\cdot ; \omega^{W}\right)\left[\widehat{F}_{s}^{t, \omega^{B}}\right]\left(\omega^{W}\right) d s .
$$

To sum up, we have obtained that for $\mathbb{P}$-a.e. $\omega \in \Omega$

$$
\begin{aligned}
y_{t}^{\mathbb{P}}\left(\omega^{B}, \omega^{W}\right)= & \mathbb{E}^{\mathbb{P}_{B}^{t, \omega^{B}}\left(\cdot ; \omega^{W}\right)}\left[\xi^{t, \omega^{B}}\right]+\left(\int_{t}^{T} \mathbb{E}^{\mathbb{P}_{B}(\cdot ; \cdot)}\left[g_{s} \mid \mathcal{F}_{t}^{B}\right]\left(\omega^{B}, \cdot\right) \cdot d \overleftarrow{W}_{s}\right)\left(\omega^{W}\right) \\
& +\int_{t}^{T} \mathbb{E}^{\mathbb{P}_{B}^{t, \omega^{B}}\left(\cdot ; \omega^{W}\right)}\left[\widehat{F}_{s}^{t, \omega^{B}}\right]\left(\omega^{W}\right) d s .
\end{aligned}
$$


But, we also have (remember that by the Blumenthal $0-1$ law $y_{t}^{\mathbb{P}_{B}^{t, \omega^{B}}(\cdot) \otimes \mathbb{P}_{W}^{0}, t, \omega^{B}}$ only depends on $\omega^{W}$ ) for any $\omega^{B} \in \Omega^{B}$ and for $\mathbb{P}_{0}^{W}$-a.e. $\omega^{W} \in \Omega^{W}$

$$
\begin{aligned}
y_{t}^{\mathbb{P}_{B}^{t, \omega^{B}}(\cdot) \otimes \mathbb{P}_{W}^{0}, t, \omega^{B}}\left(\omega^{W}\right) & =\mathbb{E}^{\mathbb{P}_{B}^{t, \omega^{B}}(\cdot) \otimes \mathbb{P}_{W}^{0}}\left[\xi^{t, \omega^{B}}+\int_{t}^{T} \widehat{F}_{s}^{t, \omega^{B}} d s+\int_{t}^{T} g_{s}^{t, \omega^{B}} \cdot d \overleftarrow{W}_{s} \mid \mathcal{G}_{t}^{t}\right]\left(\omega^{W}\right) \\
& =\mathbb{E}^{\mathbb{P}_{B}^{t, \omega^{B}}(\cdot) \otimes \mathbb{P}_{W}^{0}}\left[\xi^{t, \omega^{B}}+\int_{t}^{T} \widehat{F}_{s}^{t, \omega^{B}} d s+\int_{t}^{T} g_{s}^{t, \omega^{B}} \cdot d \overleftarrow{W}_{s} \mid \mathcal{F}_{t, T}^{W}\right]\left(\omega^{W}\right) .
\end{aligned}
$$

Using the same arguments as above, we obtain

$$
\begin{aligned}
y_{t}^{\mathbb{P}_{B}^{t, \omega^{B}}(\cdot) \otimes \mathbb{P}_{W}^{0}, t, \omega^{B}}\left(\omega^{W}\right)= & \mathbb{E}^{\mathbb{P}_{B}^{t, \omega^{B}}\left(\cdot, \omega^{W}\right)}\left[\xi^{t, \omega^{B}}\right]+\int_{t}^{T} \mathbb{E}^{\mathbb{P}_{B}^{t, \omega^{B}}\left(\cdot, \omega^{W}\right)}\left[\widehat{F}_{s}^{t, \omega^{B}}\right]\left(\omega^{W}\right) d s \\
& +\left(\int_{t}^{T} \mathbb{E}^{\mathbb{P}_{B}^{t, \omega^{B}}(\cdot, \cdot)}\left[g_{s}^{t, \omega^{B}}\right] \cdot d \overleftarrow{W}_{s}\right)\left(\omega^{W}\right)
\end{aligned}
$$

which proves the desired result.

Step 2: Since we are in a Lipschitz setting, solutions to BDSDEs can be constructed via Picard iterations. Hence, using Step 1, the results holds at each step of the iteration and therefore also when passing to the limit. We emphasize that this step crucially relies on (4.1).

\section{A.3. Proof of Lemma 4.4}

For each $\mathbb{P} \in \mathcal{P}$, let $\left(\overline{\mathcal{Y}}^{\mathbb{P}}(T, \xi), \overline{\mathcal{Z}}^{\mathbb{P}}(T, \xi)\right)$ be the solution of the BDSDE with generators $\widehat{F}$ and $g$, and terminal condition $\xi$ at time $T$. We define $\widetilde{V}^{\mathbb{P}}:=V-\overline{\mathcal{Y}}^{\mathbb{P}}(T, \xi)$. Then, $\widetilde{V}^{\mathbb{P}} \geq 0, \mathbb{P}-$ a.s. For any $0 \leq t_{1} \leq t_{2} \leq T$, let $\left(y^{\mathbb{P}, t_{2}}, z^{\mathbb{P}, t_{2}}\right):=\left(\mathcal{Y}^{\mathbb{P}}\left(t_{2}, V_{t_{2}}\right), \mathcal{Z}^{\mathbb{P}}\left(t_{2}, V_{t_{2}}\right)\right)$. Note that

$$
\mathcal{Y}_{t_{1}}^{\mathbb{P}}\left(t_{2}, V_{t_{2}}\right)(\omega)=\mathcal{Y}_{t_{1}}^{\mathbb{P}, t_{1}, \omega}\left(t_{2}, V_{t_{2}}^{t_{1}, \omega}\right), \mathbb{P}-\text { a.s. }
$$

Then by the dynamic programming principle (Theorem 4.1) we get

$$
V_{t_{1}} \geq y_{t_{1}}^{\mathbb{P}, t_{2}}, \mathbb{P}-\text { a.s. }
$$

Denote $\widetilde{y}_{t}^{\mathbb{P}, t_{2}}:=y_{t}^{\mathbb{P}, t_{2}}-\overline{\mathcal{Y}}_{t}^{\mathbb{P}}, \widetilde{z}_{t}^{\mathbb{P}, t_{2}}:=\widehat{a}_{t}^{-1 / 2}\left(z_{t}^{\mathbb{P}, t_{2}}-\overline{\mathcal{Z}}_{t}^{\mathbb{P}}\right)$. Then $\left(\widetilde{y}^{\mathbb{P}, t_{2}}, \widetilde{z}^{\mathbb{P}, t_{2}}\right)$ is solution of the following BDSDE on $\left[0, t_{2}\right]$

$$
\widetilde{y}_{t}^{\mathbb{P}, t_{2}}=\widetilde{V}_{t}^{\mathbb{P}}+\int_{t}^{t_{2}} f_{s}^{\mathbb{P}}\left(\widetilde{y}_{s}^{\mathbb{P}, t_{2}}, \widetilde{z}_{s}^{\mathbb{P}, t_{2}}\right) d s+\int_{t}^{t_{2}} \widehat{g}_{s}^{\mathbb{P}}\left(\widetilde{y}_{s}^{\mathbb{P}, t_{2}}, \widetilde{z}_{s}^{\mathbb{P}, t_{2}}\right) \cdot d \overleftarrow{W}_{s}-\int_{t}^{t_{2}} \widetilde{z}_{s}^{\mathbb{P}, t_{2}} \cdot \widehat{a}_{s}^{1 / 2} d B_{s}
$$

where

$$
\begin{aligned}
& f_{t}^{\mathbb{P}}(\omega, y, z):=\widehat{F}_{t}\left(\omega, y+\overline{\mathcal{Y}}_{t}^{\mathbb{P}}(\omega), \widehat{a}^{1 / 2}(\omega) z+\overline{\mathcal{Z}}_{t}^{\mathbb{P}}(\omega)\right)-\widehat{F}_{t}\left(\omega, \overline{\mathcal{Y}}_{t}^{\mathbb{P}}(\omega), \overline{\mathcal{Z}}_{t}^{\mathbb{P}}(\omega)\right) \\
& \widehat{g}_{t}^{\mathbb{P}}(\omega, y, z):=g_{t}\left(\omega, y+\overline{\mathcal{Y}}_{t}^{\mathbb{P}}(\omega), \widehat{a}^{1 / 2}(\omega) z+\overline{\mathcal{Z}}_{t}^{\mathbb{P}}(\omega)\right)-g_{t}\left(\omega, \overline{\mathcal{Y}}_{t}^{\mathbb{P}}(\omega), \overline{\mathcal{Z}}_{t}^{\mathbb{P}}(\omega)\right) .
\end{aligned}
$$

Then $\widetilde{V}_{t_{1}}^{\mathbb{P}} \geq \widetilde{y}_{t_{1}}^{\mathbb{P}, t_{2}}$. Therefore, $\widetilde{V}^{\mathbb{P}}$ is a positive weak doubly $f^{\mathbb{P}}$-super-martingale under $\mathbb{P}$ by Definition B.2 (given below in the Appendix).

Now, we assume that the coefficient $g$ does not depend in $(y, z)$, then obviously we have that $\bar{V}_{t_{1}}^{\mathbb{P}} \geq \bar{y}_{t_{1}}^{\mathbb{P}, t_{2}}$ where

$$
\bar{y}_{t}:=\widetilde{y}_{t}+\int_{0}^{t} \widehat{g}_{s} \cdot d \overleftarrow{W}_{s} \text { and } \bar{V}_{t}:=\widetilde{V}_{t}+\int_{0}^{t} \widehat{g}_{s} \cdot d \overleftarrow{W}_{s}
$$


Thanks to this change of variable, we have that $\left(\bar{y}^{\mathbb{P}, t_{2}}, \bar{z}^{\mathbb{P}, t_{2}}\right)$ solves the following standard BSDE on $\left[0, t_{2}\right]$

$$
\bar{y}_{t}^{\mathbb{P}, t_{2}}=\bar{V}_{t}^{\mathbb{P}}+\int_{t}^{t_{2}} \bar{f}_{s}^{\mathbb{P}}\left(\widetilde{y}_{s}^{\mathbb{P}, t_{2}}, \widetilde{z}_{s}^{\mathbb{P}, t_{2}}\right) d s-\int_{t}^{t_{2}} \bar{z}_{s}^{\mathbb{P}, t_{2}} \cdot \widehat{a}_{s}^{1 / 2} d B_{s}
$$

where

$$
\bar{f}_{t}^{\mathbb{P}}(\omega, y, z):=f_{t}\left(\omega, y+\int_{0}^{t} \widehat{g}_{s} \cdot d \overleftarrow{W}_{s}, \widehat{a}^{1 / 2}(\omega) z\right)
$$

Now applying the down-crossing inequality for $f$-martingale Theorem 6 in [16] combined with the result concerning the classical down-crossing inequality for non necessarily positive super-martingales in [24] (chapter III, p. 446), we deduce that for $\mathbb{P}-$ a.e. $\omega$, the limit $\lim _{r \in \mathbb{Q} \cap(t, T], r \downarrow t} \bar{V}_{r}^{\mathbb{P}}$, and consequently the limit $\lim _{r \in \mathbb{Q} \cap(t, T], r \downarrow t} \widetilde{V}_{r}^{\mathbb{P}}$ exists for all $t \in[0, T]$. Note that $y^{\mathbb{P}}$ is continuous, $\mathbb{P}-$ a.s., and obviously $\bar{y}^{\mathbb{P}}$ is continuous, $\mathbb{P}-$ a.s. Therefore, we get that the $\varlimsup$ im in the definition of $V^{+}$is in fact a true limit, which implies that

$$
V_{t}^{+}=\lim _{r \in \mathbb{Q} \cap(t, T], r \downarrow t} V_{r}, \mathcal{P}-q . s .,
$$

and thus $V^{+}$is càdlàg $\mathcal{P}-q . s$. Finally, we can prove the general case when $g$ depend in $(y, z)$ using classically the Banach fixed point theorem.

\section{Appendix B: Doubly $f$-supersolution and martingales}

In this section, we extend some of the results of Peng [55] concerning $f$-super-solutions of BSDEs to the case of BDSDEs. In the following, we fix a probability measure $\mathbb{P} \in \mathcal{P}$ and work implicitly with $\overline{\mathbb{F}}^{\mathbb{P}}$ and $\mathbb{F}^{W}$. We introduce the following spaces for a fixed probability $\mathbb{P}$.

- $L^{2}(\mathbb{P})$ denotes the space of all $\mathcal{F}_{T}$-measurable scalar r.v. $\xi$ with $\|\xi\|_{L^{2}}^{2}:=\mathbb{E}^{\mathbb{P}}\left[|\xi|^{2}\right]<+\infty$.

- $\mathbb{D}^{2}(\mathbb{P})$ denotes the space of $\mathbb{R}$-valued processes $Y$, s.t. $Y_{t}$ is $\mathcal{F}_{t}$ measurable for every $t \in[0, T]$, with càdlàg paths, and $\|Y\|_{\mathbb{D}^{2}(\mathbb{P})}^{2}:=\mathbb{E}^{\mathbb{P}}\left[\sup _{0 \leq t \leq T}\left|Y_{t}\right|^{2}\right]<+\infty$.

- $\mathbb{H}^{2}(\mathbb{P})$ denotes the space of all $\mathbb{R}^{d}$-valued processes $Z$ s.t. $Z_{t}$ is $\mathcal{F}_{t}$ measurable for a.e. $t \in[0, T]$, with

$$
\|Z\|_{\mathbb{H}^{2}(\mathbb{P})}^{2}:=\mathbb{E}^{\mathbb{P}}\left[\left(\int_{0}^{T}\left\|\widehat{a}_{t}^{1 / 2} Z_{t}\right\|^{2} d t\right)\right]<+\infty .
$$

Let us be given the following objects

(i) a terminal condition $\xi$ which is $\mathcal{F}_{T}$-measurable and in $L^{2}(\mathbb{P})$.

(ii) two maps $f: \Omega \times \mathbb{R} \times \mathbb{R}^{d} \rightarrow \mathbb{R}, g: \Omega \times \mathbb{R} \times \mathbb{R}^{d} \rightarrow \mathbb{R}^{l}$ verifying

- $\mathbb{E}\left[\int_{0}^{T}|f(t, 0,0)|^{2} d t\right]<+\infty$, and $\mathbb{E}\left[\int_{0}^{T}\|g(t, 0,0)\|^{2} d t\right]<+\infty$.

- There exist $(\mu, \alpha) \in \mathbb{R}_{+}^{*} \times(0,1)$ s.t. for any $\left(\omega, t, y_{1}, y_{2}, z_{1}, z_{2}\right) \in \Omega \times[0, T] \times \mathbb{R}^{2} \times\left(\mathbb{R}^{d}\right)^{2}$

$$
\begin{aligned}
\left|f\left(t, \omega, y_{1}, z_{1}\right)-f\left(t, \omega, y_{2}, z_{2}\right)\right| & \leq \mu\left(\left|y_{1}-y_{2}\right|+\left\|z_{1}-z_{2}\right\|\right), \\
\left\|g\left(t, \omega, y_{1}, z_{1}\right)-g\left(t, \omega, y_{2}, z_{2}\right)\right\|^{2} & \leq c\left|y_{1}-y_{2}\right|^{2}+\alpha\left\|z_{1}-z_{2}\right\|^{2} .
\end{aligned}
$$

(iii) a real-valued càdlàg, progressively measurable process $\left\{V_{t}, 0 \leq t \leq T\right\}$ with

$$
\mathbb{E}\left[\sup _{0 \leq t \leq T}\left|V_{t}\right|^{2}\right]<+\infty
$$


We want to study the following problem: to find a pair of processes $(y, z) \in \mathbb{D}^{2}(\mathbb{P}) \times \mathbb{H}^{2}(\mathbb{P})$ satisfying

$$
y_{t}=\xi_{T}+\int_{t}^{T} f_{s}\left(y_{s}, z_{s}\right) d s+\int_{t}^{T} g_{s}\left(y_{s}, z_{s}\right) \cdot d \overleftarrow{W}_{s}+V_{T}-V_{t}-\int_{t}^{T} z_{s} \cdot d B_{s}, \mathbb{P}-\text { a.s. }
$$

We have the following existence and uniqueness theorem

Proposition B.1. Under the above hypothesis there exists a unique pair of processes $(y, z) \in \mathbb{D}^{2}(\mathbb{P}) \times \mathbb{H}^{2}(\mathbb{P})$ solution of BDSDE (B.1).

Proof. In the case where $V \equiv 0$, the proof can be found in [52]. Otherwise, we can make the change of variable $\bar{y}_{t}:=y_{t}+V_{t}$ and treat the equivalent BDSDE

$$
\bar{y}_{t}=\xi_{T}+V_{T}+\int_{t}^{T} f_{s}\left(\bar{y}_{s}-V_{s}, z_{s}\right) d s+\int_{t}^{T} g_{s}\left(\bar{y}_{s}-V_{s}, z_{s}\right) \cdot d \overleftarrow{W}_{s}-\int_{t}^{T} z_{s} \cdot d B
$$

We also have a comparison theorem in this context

Proposition B.2. Let $\xi_{1}$ and $\xi_{2} \in L^{2}(\mathbb{P}), V^{i}, i=1,2$ be two adapted càdlàg processes and $f_{s}^{i}(y, z), g_{s}^{i}(y, z)$ four functions verifying the above assumption. Let $\left(y^{i}, z^{i}\right) \in \mathbb{D}^{2}(\mathbb{P}) \times \mathbb{H}^{2}(\mathbb{P}), i=1,2$ be the solution of the following BDSDEs

$$
y_{t}^{i}=\xi_{T}^{i}+\int_{t}^{T} f_{s}^{i}\left(y_{s}^{i}, z_{s}^{i}\right) d s+\int_{t}^{T} g_{s}\left(y_{s}^{i}, z_{s}^{i}\right) \cdot d \overleftarrow{W}_{s}+V_{T}^{i}-V_{t}^{i}-\int_{t}^{T} z_{s}^{i} \cdot d B_{s}, \mathbb{P}-a . s,
$$

respectively. If we have $\mathbb{P}$ - a.s. that $\xi_{1} \geq \xi_{2}, V^{1}-V^{2}$ is non decreasing, and $f_{s}^{1}\left(y_{s}^{1}, z_{s}^{1}\right) \geq f_{s}^{2}\left(y_{s}^{1}, z_{s}^{1}\right)$ then it holds that for all $t \in[0, T]$

$$
y_{t}^{1} \geq y_{t}^{2}, \mathbb{P}-\text { a.s. }
$$

For a given $\mathbb{G}$-stopping time, we now consider the following BDSDE

$$
y_{t}=\xi_{T}+\int_{t \wedge \tau}^{\tau} f_{s}\left(y_{s}, z_{s}\right) d s+\int_{t \wedge \tau}^{\tau} g_{s}\left(y_{s}, z_{s}\right) \cdot d \overleftarrow{W}_{s}+V_{\tau}-V_{t \wedge \tau}-\int_{t \wedge \tau}^{\tau} z_{s} \cdot d B_{s}, \mathbb{P}-a . s .
$$

where $\xi \in L^{2}(\mathbb{P})$ and $V \in \mathbb{I}^{2}(\mathbb{P})$.

Definition B.1. If $y$ is a solution of BDSDE of form (B.3), the we call $y$ a doubly $f$-super-solution on $[0, \tau]$. If $V \equiv 0$ in $[0, \tau]$, then we call $y$ a doubly $f$-solution.

We now introduce the notion of doubly $f$-(super)martingales.

\section{Definition B.2.}

(i) A doubly $f$-martingale on $[0, T]$ is a doubly $f$-solution on $[0, T]$.

(ii) A process $\left(Y_{t}\right)$ is a doubly $f$-super-martingale in the strong (resp. weak) sense if for all stopping time $\tau \leq t$ (resp. all $t \leq T)$, we have $\mathbb{E}^{\mathbb{P}}\left[\left|Y_{\tau}\right|^{2}\right]<+\infty$ (resp. $\left.\mathbb{E}^{\mathbb{P}}\left[\left|Y_{t}\right|^{2}\right]<+\infty\right)$ and if the doubly f-solution $\left(y_{s}\right)$ on $[0, \tau]$ (resp. $[0, t]$ ) with terminal condition $Y_{\tau}\left(\right.$ resp. $Y_{t}$ ) verifies $y_{\sigma} \leq Y_{\sigma}$ for every stopping time $\sigma \leq \tau$ (resp. $y_{s} \leq Y_{s}$ for every $s \leq t)$.

\section{Appendix C: Reflected backward doubly stochastic differential equations}

In this section, we want to study the problem of a reflected backward doubly stochastic differential equation (RBDSDE for short) with one càdlàg barrier. This is an extension of the work of Hamadène and Ouknine [34] for the standard reflected BSDEs to our case. So in addition to the terminal condition and generators that we used in the previous section, we need 
(iv) a barrier $\left\{S_{t}, 0 \leq t \leq T\right\}$, which is a real-valued càdlàg $\mathcal{F}_{t}$-measurable process satisfying $S_{T} \leq \xi$ and

$$
\mathbb{E}\left[\sup _{0 \leq t \leq T}\left(S_{t}^{+}\right)^{2}\right]<+\infty .
$$

Now we present the definition of the solution of RBDSDEs with one lower barrier.

Definition C.1. We call $(Y, Z, K)$ a solution of the backward doubly stochastic differential equation with one reflecting lower barrier $S($.$) , terminal condition \xi$ and coefficients $f$ and $g$, if the following holds:

(i) $Y \in \mathbb{D}^{2}(\mathbb{P}), Z \in \mathbb{H}^{2}(\mathbb{P})$.

(ii) $Y_{t}=\xi+\int_{t}^{T} f\left(s, Y_{s}, Z_{s}\right) d s+\int_{t}^{T} g\left(s, Y_{s}, Z_{s}\right) \cdot d \overleftarrow{W}_{s}-\int_{t}^{T} Z_{s} \cdot d B_{s}+K_{T}-K_{t}, 0 \leq t \leq T$.

(iii) $Y_{t} \geq S_{t}, 0 \leq t \leq T$, a.s.

(iv) If $K^{c}\left(\right.$ resp. $\left.K^{d}\right)$ is the continuous (resp. purely discontinuous) part of $K$, then

$$
\int_{0}^{T}\left(Y_{s}-S_{s}\right) d K_{s}^{c}=0, \text { a.s. and } \forall t \leq T, \Delta K_{t}^{d}=\left(S_{t^{-}}-Y_{t}\right)^{+} \mathbf{1}_{\left[Y_{t^{-}}=S_{t^{-}}\right]} .
$$

Remark C.1. The condition (iv) implies in particular that $\int_{0}^{T}\left(Y_{s^{-}}-S_{s^{-}}\right) d K_{s}=0$. Actually

$$
\begin{aligned}
\int_{0}^{T}\left(Y_{s^{-}}-S_{s^{-}}\right) d K_{s} & =\int_{0}^{T}\left(Y_{s^{-}}-S_{s^{-}}\right) d K_{s}^{c}+\int_{0}^{T}\left(Y_{s^{-}}-S_{s^{-}}\right) d K_{s}^{d} \\
& =\int_{0}^{T}\left(Y_{s^{-}}-S_{s}\right) d K_{s}^{c}+\sum_{s \leq T}\left(Y_{s^{-}}-S_{s^{-}}\right) \Delta K_{s}^{d}=0 .
\end{aligned}
$$

The last term of the second equality is null since $K^{d}$ jumps only when $Y_{s^{-}}=S_{s^{-}}$.

The main objective of this section is to prove the following theorem.

Theorem C.1. Under the above hypotheses, the RBDSDE in Definition C.1 has a unique solution $(Y, Z, K)$.

Before we start proving this theorem, let us establish the same result in the case where $f$ and $g$ do not depend on $y$ and $z$. More precisely, given $f$ and $g$ such that

$$
\mathbb{E}\left[\int_{0}^{T}|f(s)|^{2} d s\right]+\mathbb{E}\left[\int_{0}^{T}\|g(s)\|^{2} d s\right]<+\infty
$$

and $\xi$ as above, consider the reflected BDSDE

$$
Y_{t}=\xi+\int_{t}^{T} f(s) d s+\int_{t}^{T} g(s) \cdot d \overleftarrow{W}_{s}-\int_{t}^{T} Z_{s} \cdot d B_{s}+K_{T}-K_{t}
$$

Proposition C.1. There exists a unique triplet $(Y, Z, K)$ verifies conditions of Definition C.1 and satisfies (C.1).

Proof. a) Existence: The method combines penalization and the Snell envelope method. For each $n \in \mathbb{N}^{*}$, we set

$$
f_{n}(s, y)=f(s)+n\left(y_{s}-S_{s}\right)^{-},
$$

and consider the BDSDE

$$
Y_{t}^{n}=\xi^{n}+\int_{t}^{T} f_{n}\left(s, Y_{s}^{n}\right) d s+\int_{t}^{T} g(s) \cdot d \overleftarrow{W}_{s}-\int_{t}^{T} Z_{s}^{n} \cdot d B_{s}
$$


It is well known (see Pardoux and Peng [52]) that $\operatorname{BDSDE}(\mathrm{C} .2)$ has a unique solution $\left(Y^{n}, Z^{n}\right) \in \mathbb{D}^{2}(\mathbb{P}) \times \mathbb{H}^{2}(\mathbb{P})$ such that for each $n \in \mathbb{N}$,

$$
\mathbb{E}\left[\sup _{0 \leq t \leq T}\left|Y_{t}^{n}\right|^{2}+\int_{0}^{T}\left\|Z_{s}^{n}\right\|^{2} d s\right]<+\infty .
$$

From now on the proof will be divided into three steps.

Step 1: For all $n \geq 0$ and $(s, y) \in[0, T] \times \mathbb{R}$,

$$
f_{n}(s, y, z) \leq f_{n+1}(s, y, z),
$$

which provide by the comparison theorem, $Y_{t}^{n} \leq Y_{t}^{n+1}, t \in[0, T]$ a.s. For each $n \in \mathbb{N}$, denoting

$$
\bar{Y}_{t}^{n}:=Y_{t}^{n}+\int_{0}^{t} g(s) d \overleftarrow{W}_{s}, \bar{\xi}:=\xi+\int_{0}^{T} g(s) \cdot d \overleftarrow{W}_{s}, \bar{S}_{t}:=S_{t}+\int_{0}^{t} g(s) \cdot d \overleftarrow{W}_{s}
$$

we have

$$
\bar{Y}_{t}^{n}=\bar{\xi}+\int_{t}^{T} f(s) d s+n \int_{t}^{T}\left(\bar{Y}_{s}^{n}-\bar{S}_{s}\right)^{-} d s-\int_{t}^{T} Z_{s}^{n} \cdot d B_{s}
$$

The process $\bar{Y}_{t}^{n}$ satisfies

$$
\forall t \leq T, \bar{Y}_{t}^{n}=\underset{\tau \geq t}{\operatorname{ess~sup}} \mathbb{E}\left[\int_{t}^{\tau} f(s) d s+\left(\bar{Y}_{\tau}^{n} \wedge \bar{S}_{\tau}\right) \mathbf{1}_{\{\tau<T\}}+\bar{\xi} \mathbf{1}_{\{\tau=T\}} \mid \mathcal{G}_{t}\right]
$$

In fact, for any $n \in \mathbb{N}$ and $t \leq T$ we have

$$
\bar{Y}_{t}^{n}=\bar{\xi}+\int_{t}^{T} f(s) d s+n \int_{t}^{T}\left(\bar{Y}_{s}^{n}-\bar{S}_{s}\right)^{-} d s-\int_{t}^{T} \bar{Z}_{s}^{n} \cdot d B_{s}
$$

Therefore for any $\mathbb{G}$-stopping time $\tau \geq t$ we have

$$
\begin{aligned}
\bar{Y}_{t}^{n} & =\mathbb{E}\left[\bar{Y}_{\tau}^{n}+\int_{t}^{\tau} f(s) d s+n \int_{t}^{\tau}\left(\bar{Y}_{s}^{n}-\bar{S}_{s}\right)^{-} d s \mid \mathcal{G}_{t}\right] \\
& \geq \mathbb{E}\left[\left(\bar{S}_{\tau} \wedge \bar{Y}_{\tau}^{n}\right) \mathbf{1}_{[\tau<T]}+\bar{\xi} \mathbf{1}_{\{\tau=T\}}+\int_{t}^{\tau} f(s) d s \mid \mathcal{G}_{t}\right],
\end{aligned}
$$

since $\bar{Y}_{\tau}^{n} \geq\left(\bar{S}_{\tau} \wedge \bar{Y}_{\tau}^{n}\right) \mathbf{1}_{[\tau<T]}+\bar{\xi} \mathbf{1}_{\{\tau=T\}}$. On the other hand, let $\tau_{t}^{*}$ be the stopping time defined as follows:

$$
\tau_{t}^{*}=\inf \left\{s \geq t, \bar{K}_{s}^{n}-\bar{K}_{t}^{n}>0\right\} \wedge T,
$$

where $\bar{K}_{t}^{n}=n \int_{0}^{t}\left(\bar{Y}_{s}^{n}-\bar{S}_{s}\right)^{-} d s$. Let us show that $\left.\mathbf{1}_{\left[\tau_{t}^{*}<T\right]} \bar{Y}_{\tau_{t}^{*}}^{n}\right)=\left(\bar{S}_{\tau_{t}^{*}} \wedge \bar{Y}_{\tau_{t}^{*}}^{n}\right) \mathbf{1}_{\left[\tau_{t}^{*}<T\right]}$.

Let $\omega$ be fixed such that $\tau_{t}^{*}(\omega)<T$. Then there exists a sequence $\left(t_{k}\right)_{k \geq 0}$ of real numbers which decreases to $\tau_{t}^{*}(\omega)$ such that $\bar{Y}_{t_{k}}^{n}(\omega) \leq \bar{S}_{t_{k}}(\omega)$. As $\bar{Y}^{n}$ and $\bar{S}$ are RCLL processes then taking the limit as $k \rightarrow \infty$ we obtain $\bar{Y}_{\tau_{t}^{*}}^{n} \leq \bar{S}_{\tau_{t}^{*}}$ which implies $\left.\mathbf{1}_{\left[\tau_{t}^{*}<T\right]} \bar{Y}_{\tau_{t}^{*}}^{n}\right)=\left(\bar{S}_{\tau_{t}^{*}} \wedge \bar{Y}_{\tau_{t}^{*}}^{n}\right) \mathbf{1}_{\left[\tau_{t}^{*}<T\right]}$. Now from (C.5), we deduce that:

$$
\begin{aligned}
\bar{Y}_{t}^{n} & =\bar{Y}_{\tau_{t}^{*}}^{n}+\int_{t}^{\tau_{t}^{*}} f(s) d s-\int_{t}^{\tau_{t}^{*}} \bar{Z}_{s}^{n} \cdot d B_{s} \\
& =\left(\bar{S}_{\tau_{t}^{*}} \wedge \bar{Y}_{\tau_{t}^{*}}^{n}\right) \mathbf{1}_{\left[\tau_{t}^{*}<T\right]}+\bar{\xi} \mathbf{1}_{\left\{\tau_{t}^{*}=T\right\}}+\int_{t}^{\tau_{t}^{*}} f(s) d s-\int_{t}^{\tau_{t}^{*}} \bar{Z}_{s}^{n} \cdot d B_{s} .
\end{aligned}
$$

Taking the conditional expectation and using inequality (C.6) we obtain: $\forall n \geq 0$, and $t \geq T$

$$
\bar{Y}_{t}^{n}=\underset{\tau \geq t}{\operatorname{ess} \sup } \mathbb{E}\left[\int_{t}^{\tau} f(s) d s+\left(\bar{Y}_{\tau}^{n} \wedge \bar{S}_{\tau}\right) \mathbf{1}_{\{\tau<T\}}+\bar{\xi} \mathbf{1}_{\{\tau=T\}} \mid \mathcal{G}_{t}\right] .
$$

Step 2: There exists a RCLL $\left(Y_{t}\right)_{t \leq T}$ of $\mathbb{D}^{2}(\mathbb{P})$ such that $\mathbb{P}-$ a.s. 
(i) $Y=\lim _{n \rightarrow \infty} Y^{n}$ in $\mathbb{H}^{2}(\mathbb{P}), S \leq Y$.

(ii) for any $t \leq T$,

$$
Y_{t}=\underset{\tau \geq t}{\operatorname{esssup}} \mathbb{E}\left[\int_{t}^{\tau} f(s) d s+\bar{S}_{\tau} \mathbf{1}_{\{\tau<T\}}+\bar{\xi} \mathbf{1}_{\{\tau=T\}} \mid \mathcal{G}_{t}\right]-\int_{0}^{t} g(s) \cdot d \overleftarrow{W}_{s}
$$

Actually for $t \leq T$ let us set

$$
\tilde{Y}_{t}:=\underset{\tau \geq t}{\operatorname{esssup}} \mathbb{E}\left[\int_{t}^{\tau} f(s) d s+\bar{S}_{\tau} \mathbf{1}_{\{\tau<T\}}+\bar{\xi} \mathbf{1}_{\{\tau=T\}} \mid \mathcal{G}_{t}\right] .
$$

since $\bar{S} \in \mathbb{D}^{2}(\mathbb{P}), f \in \mathbb{H}^{2}(\mathbb{P})$ and $\bar{\xi}$ is square integrable, the process $\tilde{Y}$ belongs to $\mathbb{D}^{2}(\mathbb{P})$. On the other hand for any $n \geq 0$ and $t \leq T$ we have $\bar{Y}_{t}^{n} \leq \tilde{Y}_{t}$. Thus there exist a $\mathbb{G}$-progressively measurable process $\bar{Y}$ such that $\mathbb{P}-$ a.s., for any $t \leq T, \bar{Y}_{t}^{n} \nearrow \bar{Y}_{t} \leq \tilde{Y}_{t}$ and we have $Y_{t}^{n} \nearrow Y_{t}=\bar{Y}_{t}-\int_{0}^{t} g(s) \cdot d \overleftarrow{W}_{s}$, then $Y=\lim _{n \rightarrow \infty} Y^{n}$ in $\mathbb{H}^{2}(\mathbb{P})$

Besides, the process $\bar{Y}^{n}+\int_{0}^{.} f(s) d s$ is a càdlàg super-martingale as the Snell envelope of

$$
\left(\int_{0}^{\cdot} f(s) d s+\bar{S} \cdot \wedge \bar{Y}_{\cdot}^{n}\right) \mathbf{1}_{[\cdot<T]}+\bar{\xi} \mathbf{1}_{\{\cdot=T\}}
$$

and it converges increasingly to $\bar{Y} .+\int_{0}^{\cdot} f(s) d s$. It follows that the latter process is a càdlàg super-martingale. Hence, the process $Y$ is also $\mathbb{G}$-progressively measurable, càdlàg, and belongs to $\mathbb{D}^{2}(\mathbb{P})$. Even more than that, $Y_{t}$ is $\mathcal{F}_{t}$-measurable for every $t \in[0, T]$ as the limit of $Y_{t}^{n}$, which has this property.

Next let us prove that $Y \geq S$. We have

$$
\mathbb{E}\left[Y_{0}^{n}\right]=\mathbb{E}\left[\xi+\int_{0}^{T} f(s) d s\right]+\mathbb{E}\left[\int_{0}^{T} n\left(Y_{s}^{n}-S_{s}\right)^{-} d s\right] .
$$

Dividing the two sides by $n$ and taking the limit as $n \rightarrow \infty$, we obtain

$$
\mathbb{E}\left[\int_{0}^{T}\left(Y_{s}-S_{s}\right)^{-} d s\right]=0 .
$$

Since the processes $Y$ and $S$ are càdlàg, then, $\mathbb{P}-$ a.s., $Y_{t} \geq S_{t}$, for $t<T$. But $Y_{T}=\xi \geq S_{T}$, therefore $Y \geq S$. Finally let us show that $Y$ satisfies (C.8). But this is a direct consequence of the continuity of the Snell envelope through sequences of increasing càdlàg processes. In fact on the one hand, the sequence of increasing càdlàg processes $\left.\left(\left(\bar{S}_{t} \wedge \bar{Y}_{t}^{n}\right) \mathbf{1}_{[t<T]}+\bar{\xi} \mathbf{1}_{\{t=T\}}\right)_{t \leq T}\right)_{t \leq T}$ converges increasingly to the càdlàg process $\left(\bar{S}_{t} \mathbf{1}_{[t<T]}+\right.$ $\left.\left.\bar{\xi} \mathbf{1}_{\{t=T\}}\right)_{t \leq T}\right)\left[t \leq T\right.$ since $\bar{Y}_{t} \geq \bar{S}_{t}$. Therefore,

$$
\int_{0}^{t} f(s) d s+\bar{Y}_{t}^{n} \longrightarrow \underset{\tau \geq t}{\operatorname{esssup}} \mathbb{E}\left[\int_{0}^{\tau} f(s) d s+\bar{S}_{\tau} \mathbf{1}_{\{\tau<T\}}+\bar{\xi} \mathbf{1}_{\{\tau=T\}} \mid \mathcal{G}_{t}\right]=\int_{0}^{t} f(s) d s+\bar{Y}_{t},
$$

which implies that

$$
Y_{t}=\bar{Y}_{t}-\int_{0}^{t} g(s) \cdot d \overleftarrow{W}_{s}=\underset{\tau \geq t}{\operatorname{essup}} \mathbb{E}\left[\int_{t}^{\tau} f(s) d s+\bar{S}_{\tau} \mathbf{1}_{\{\tau<T\}}+\bar{\xi} \mathbf{1}_{\{\tau=T\}} \mid \mathcal{G}_{t}\right]-\int_{0}^{t} g(s) \cdot d \overleftarrow{W}_{s}
$$

Step 3: We know from (C.8) that the process $\int_{0}^{\cdot} f(s) d s+\bar{Y}_{\cdot}^{n}$ is a Snell envelope. Then, there exist a process $K \in \mathbb{I}^{2}(\mathbb{P})$ and a $\mathbb{G}$-martingale such that

$$
\int_{0}^{t} f(s) d s+Y_{t}+\int_{0}^{t} g(s) \cdot d \overleftarrow{W}_{s}=M_{t}-K_{t}, 0 \leq t \leq T
$$


Additionally $K=K^{c}+K^{d}$ where $K^{c}$ is continuous, non-decreasing and $K^{d}$ non-decreasing purely discontinuous predictable such that for any $t \leq T, \Delta_{t} K^{d}=\left(S_{t^{-}}-Y_{t}\right) 1_{\left\{Y_{t^{-}}=S_{t^{-}}\right\}}$. Now the martingale $M$ belongs to $\mathbb{D}^{2}(\mathbb{P})$, so that the Itô's martingale representation theorem implies the existence of a $\mathbb{G}$-predictable process $Z \in \mathbb{H}^{2}(\mathbb{P})$ such that

$$
M_{t}=M_{0}+\int_{0}^{t} Z_{s} \cdot d B_{s}, \quad 0 \leq t \leq T, \mathbb{P}-a . s .
$$

Hence

$$
Y_{t}=Y_{0}-\int_{0}^{t} f(s) d s-\int_{0}^{t} g(s) \cdot d \overleftarrow{W}_{s}+\int_{0}^{t} Z_{s} \cdot d B_{s}-K_{t}, 0 \leq t \leq T
$$

The proof of $\int_{0}^{T}\left(Y_{s}-S_{s}\right) d K_{s}^{c}=0$ is the same as in [34], so we omit it.

It remains to show that $Z_{t}$ and $K_{t}$ are in fact $\mathcal{F}_{t}$-measurable. For $K_{t}$, it is obvious since it is the limit of $K_{t}^{n}=\int_{0}^{t} n\left(Y_{s}^{n}-S_{s}\right)^{-} d s$ which is $\mathcal{F}_{t}-$ measurable for each $t \leq T$. Now

$$
\int_{t}^{T} Z_{s} \cdot d B_{s}=\xi+\int_{t}^{T} f\left(s, Y_{s}, Z_{s}\right) d s+\int_{t}^{T} g\left(s, Y_{s}, Z_{s}\right) \cdot d \overleftarrow{W}_{s}-Y_{t}+K_{T}-K_{t}
$$

and the right side is $\mathcal{F}_{T}^{B} \vee \mathcal{F}_{t, T}^{W}$-measurable. Hence from the Itô's martingale representation theorem $\left(Z_{s}\right)_{t \leq s \leq T}$ is $\mathcal{F}_{s}^{B} \vee \mathcal{F}_{t, T}^{W}$ adapted. Consequently $Z_{s}$ is $\mathcal{F}_{s}^{B} \vee \mathcal{F}_{t, T}^{W}$-measurable for any $t<s$, so it is $\mathcal{F}_{s}^{B} \vee \mathcal{F}_{s, T}^{W}$-measurable.

b) Uniqueness: Under Lipschitz continuous conditions, the proof of uniqueness is standard in BSDE theory (see e.g. proof of Proposition 2.1. in [1]).

The existence of solution of RBDSDE in Theorem C.1 is obtained via a standard fixed Banach point theorem for reflected BSDEs (see for instance El Karoui, Hamadène and Matoussi [25]).

\section{References}

[1] Aman, A., And Mrhardy, N. Obstacle problem for SPDE with onlinear Neumann boundary condition via reflected generalized backward doubly SDEs. Statistics \& Probability Letters 83, 3 (2013), 863-874.

[2] Avellaneda, M., Levy, A., And Paras, A. Pricing and hedging derivative securities in markets with uncertain volatility. Applied Mathematical Finance (1995).

[3] Bachouch, A., Gobet, E., And Matoussi, A. Empirical regression method for backward doubly stochastic differential equations. SIAM/ASA Journal on Uncertainty Quantification 4, 1 (2016), 358-379.

[4] Bachouch, A., Lasmar, A. B., Matoussi, A., And Mnif, M. Numerical scheme for semilinear SPDEs via backward doubly SDEs. Stochastic Partial Differential Equations: Analysis and Computation 1, 1-43.

[5] Bally, V., And Matoussi, A. Weak solutions for SPDEs and backward doubly stochastic differential equations. Journal of Theoretical Probability 14, 1 (2001), 125-164.

[6] Bertsekas, D., And Shreve, S. Stochastic optimal control: the discrete-time case. Academic Press, New York, 1978.

[7] Bichteler, K. Stochastic integration and $L^{p}$-theory of semimartingales. Annals of Probability 9, 1 (1981), 49-89.

[8] Buckdahn, R., Bulla, I., And Ma, J. Pathwise Taylor expansions for Itō random fields. Mathematical Control and Related Fields 1, 4 (2011), 437-468.

[9] Buckdahn, R., And Ma, J. Stochastic viscosity solutions for nonlinear stochastic partial differential equations. I. Stochastic Processes and their Applications 93, 2 (2001), 181-204. 
[10] Buckdahn, R., And Ma, J. Stochastic viscosity solutions for nonlinear stochastic partial differential equations. II. Stochastic Processes and their Applications 93, 2 (2001), 205-228.

[11] Buckdahn, R., And Ma, J. Pathwise stochastic Taylor expansions and stochastic viscosity solutions for fully nonlinear stochastic PDEs. The Annals of Probability 30, 3 (2002), 1131-1171.

[12] Buckdahn, R., And Ma, J. Pathwise stochastic control problems and stochastic HJB equations. SIAM Journal on Control and Optimization 45, 6 (2007), 2224-2256.

[13] Buckdahn, R., Ma, J., And Zhang, J. Pathwise Taylor expansions for random fields on multiple dimensional paths. Stochastic Processes and their Applications 125, 7 (2015), 2820-2855.

[14] Buckdahn, R., Ma, J., And Zhang, J. Pathwise viscosity solutions of stochastic PDEs and forward path-dependent PDEs. arXiv preprint arXiv:1501.06978 (2015).

[15] Caruana, M., Friz, P., And Oberhauser, H. A (rough) pathwise approach to a class of non-linear stochastic partial differential equations. Annales de l'institut Henri Poincaré, Analyse Non Linéaire (C) 28, 1 (2011), 27-46.

[16] Chen, Z., And Peng, S. A general downcrossing inequality for $g$-martingales. Statistics 85 Probability Letters 46, 2 (2000), 169-175.

[17] Cheridito, P., Soner, H., Touzi, N., And Victoir, N. Second-order backward stochastic differential equations and fully nonlinear parabolic PDEs. Communications on Pure and Applied Mathematics 60, 7 (2007), 1081-1110.

[18] Crandall, M., Ishit, H., And Lions, P.-L. User's guide to viscosity solutions of second order partial differential equations. Bulletin of the American Mathematical Society 27, 1 (1992), 1-67.

[19] Dalang, R., Khoshnevisan, D., and Nualart, E. Hitting probabilities for systems of non-linear stochastic heat equations with additive noise. ALEA. Latin American Journal of Probability and Mathematical Statistics 3 (2007), 231-271.

[20] Dawson, D. Stochastic evolution equations. Mathematical Biosciences 15, 3 (1972), 287-316.

[21] Dellacherie, C., And Meyer, P. Probabilités et Potentiel, Chapitres XII à XVI, Théorie du potentiel. Hermann, Paris, 1980.

[22] Denis, L., And Martini, C. A theoretical framework for the pricing of contingent claims in the presence of model uncertainty. The Annals of Applied Probability 16, 2 (2006), 827-852.

[23] Dienl, J., AND Friz, P. Backward stochastic differential equations with rough drivers. The Annals of Probability 40, 4 (2012), 1715-1758.

[24] Doob, J. L. Classical potential theory and its probabilistic counterpart. Classics in Mathematics. SpringerVerlag, Berlin, 2001. Reprint of the 1984 edition.

[25] El Karoui, N., Hamadène, S., And Matoussi, A. Backward stochastic differential equations and applications. Chapter 8 in the book Indifference Pricing: Theory and Applications, Springer-Verlag (2008), 267-320.

[26] El Karoui, N., And Tan, X. Capacities, measurable selection and dynamic programming part I: abstract framework. arXiv preprint arXiv:1310.3363 (2013).

[27] El Karoui, N., and Tan, X. Capacities, measurable selection and dynamic programming part II: application in stochastic control problems. arXiv preprint arXiv:1310.3364 (2013).

[28] Fremlin, D. H. Consequences of Martin's axiom, vol. 84 of Cambridge Tracts in Mathematics. Cambridge University Press, Cambridge, 1984.

[29] Friz, P., Gassiat, P., Lions, P.-L., And Souganidis, P. Eikonal equations and pathwise solutions to fully non-linear SPDEs. arXiv preprint arXiv:1602.04746 (2016).

[30] Gerencsér, M., Gyöngy, I., And Krylov, N. On the solvability of degenerate stochastic partial 
differential equations in Sobolev spaces. Stoch. Partial Differ. Equ. Anal. Comput. 3, 1 (2015), 52-83.

[31] Gubinelli, M., Tindel, S., And Torrecilla, I. Controlled viscosity solutions of fully nonlinear rough PDEs. arXiv preprint arXiv:1403.2832 (2014).

[32] Gyöngy, I., And Krylov, N. Accelerated finite difference schemes for linear stochastic partial differential equations in the whole space. SIAM J. Math. Anal. 42, 5 (2010), 2275-2296.

[33] GyÖngy, I., And Krylov, N. Accelerated numerical schemes for PDEs and SPDEs. In Stochastic analysis 2010. Springer, Heidelberg, 2011, pp. 131-168.

[34] Hamadène, S., And Ouknine, Y. Reflected backward SDes with general jumps. Theory of Probability \& Its Applications 60, 2 (2015), 357-376.

[35] Ichikawa, A. Linear stochastic evolution equations in Hilbert space. Journal of Differential Equations 28, 2 (1978), 266-277.

[36] Karandikar, R. On pathwise stochastic integration. Stochastic Processes and Their Applications, 57:1118 (1995).

[37] Kazi-Tani, N., PossamaÏ, D., And Zhou, C. Second order BSDEs with jumps: existence and probabilistic representation for fully-nonlinear PIDEs. Electronic Journal of Probability 20 (2015).

[38] Krylov, N., And Rozovskĭ, B. On the Cauchy problem for linear stochastic partial differential equations. Izvestiya: Mathematics 11, 6 (1977), 1267-1284.

[39] Krylov, N., And Rozovskĭ, B. Stochastic evolution equations. Journal of Soviet Mathematics 16, 4 (1981), 1233-1277.

[40] Kunita, H. Stochastic flows and stochastic differential equations, vol. 24 of Cambridge Studies in Advanced Mathematics. Cambridge University Press, Cambridge, 1990.

[41] Lin, Y. A new existence result for second-order BSDEs with quadratic growth and their applications. Stochastics: An International Journal of Probability and Stochastic Processes 88, 1 (2016), 128-146.

[42] Lions, P.-L., And Souganidis, P. E. Fully nonlinear viscosity stochastic partial differential equations: non-smooth equations and applications. C.R. Acad. Sci. Paris 32\%, 1 (1998), 735-741.

[43] Lions, P.-L., And Souganidis, P. E. Équations aux dérivées partielles stochastiques nonlinéaires et solutions de viscosité. Séminaire équations aux dérivées partielles, 1998-1999, 1 (2000), 1-13.

[44] Lions, P.-L., And Souganidis, P. E. Viscosity solutions of fully nonlinear stochastic partial differential equations. Sürikaisekikenkyūsho Kökyūroku, 1287 (2002), 58-65. Viscosity solutions of differential equations and related topics (Japanese) (Kyoto, 2001).

[45] Lyons, T. J. Uncertain volatility and the risk-free synthesis of derivatives. Applied Mathematical Finance 2 (1995), 117-133.

[46] Matoussi, A., And Sheutzow, M. Semilinear stochastic PDE's with nonlinear noise and backward doubly SDE's. Journal of Theoretical Probability 15 (2002), 1-39.

[47] Nutz, M. Pathwise construction of stochastic integrals. Electronic Communications in Probability 17, 24 (2012), 1-7.

[48] Nutz, M. A quasi-sure approach to the control of non-Markovian stochastic differential equations. Electronic Journal of Probability 17, 23 (2012), 1-23.

[49] Ocone, D., And Pardoux, E. A generalized itô-ventzell formula. application to a class of anticipating stochastic differential equations. Annales de l'institut Henri Poincaré, Probabilités et Statistiques (B) 25, 1 (1989), 39-71.

[50] Pardoux, É. Stochastic partial differential equations and filtering of diffusion processes. Stochastics 3, 1-4 (1980), 127-167.

[51] Pardoux, É., And Peng, S. Adapted solution of a backward stochastic differential equation. Systems 85 
Control Letters 14, 1 (1990), 55-61.

[52] Pardoux, É., And Peng, S. Backward doubly SDE's and systems of quasilinear sPdes. Probab. Theory and Related Field 98 (1994), 209-227.

[53] Pardoux, É., And Protter, P. A two-sided stochastic integral and its calculus. Probab. Theory and Related Field 76, 1 (1987), 15-49.

[54] Peng, S. Backward SDE and related g-expectation. In Backward stochastic differential equations, N. El Karoui and L. Mazliak, Eds., vol. 364 of Pitman research notes in mathematics. Longman, Harlow, 1997, pp. 141-159.

[55] Peng, S. Monotonic limit theorem of BSDE and nonlinear decomposition theorem of Doob-Meyer's type. Probability Theory and Related Fields 113, 4 (1999), 473-499.

[56] PossamaÏ, D. Second order backward stochastic differential equations under a monotonicity condition. Stochastic Processes and their Applications 123, 5 (2013), 1521-1545.

[57] Possamaï, D., And Tan, X. Weak approximation of second-order BSDEs. The Annals of Applied Probability 25, 5 (2015), 2535-2562.

[58] PossamaÏ, D., Tan, X., And Zhou, C. Stochastic control for a class of non-linear stochastic kernels and applications. arXiv preprint arXiv:1510.08439 (2015).

[59] PossamaÏ, D., And Zhou, C. Second order backward stochastic differential equations with quadratic growth. Stochastic Process. Appl. 123, 10 (2013), 3770-3799.

[60] REN, Z., AND TAN, X. On the convergence of monotone schemes for path-dependent PDE. arXiv preprint arXiv:1504.01872 (2015).

[61] Shi, Y., Gu, Y., AND LiU, K. Comparison theorems of backward doubly stochastic differential equations and applications. Stochastic Analysis and its Applications 23, 1 (2005), 97-110.

[62] Soner, H., Touzi, N., And Zhang, J. Martingale representation theorem for the G-expectation. Stochastic Processes and their Applications 121, 2 (2011), 265-287.

[63] Soner, H., Touzi, N., And Zhang, J. Quasi-sure stochastic analysis through aggregation. Electronic Journal of Probability 16, 67 (2011), 1844-1879.

[64] Soner, H., Touzi, N., And Zhang, J. Dual formulation of second order target problems. The Annals of Applied Probability 23, 1 (2013), 308-347.

[65] Soner, H. M., Touzi, N., And Zhang, J. Wellposedness of second order backward SDEs. Probability Theory and Related Fields 153, 1-2 (2012), 149-190.

[66] Stricker, C., And Yor, M. Calcul stochastique dépendant d'un paramètre. Zeitschrift für Wahrscheinlichkeitstheorie und Verwandte Gebiete 45, 2 (1978), 109-133.

[67] Stroock, D., and Varadhan, S. Multidimensional diffusion processes. Springer, 1979. 